\title{
Cytokine-Ion Channel Interactions in Pulmonary Inflammation
}

\author{
Jürg Hamacher ${ }^{1,2,3 *}$, Yalda Hadizamani ${ }^{1,3 \dagger}$, Michèle Borgmann ${ }^{1,3 \dagger}$, Markus Mohaupt ${ }^{4}$, \\ Daniela Narcissa Männel ${ }^{5}$, Ueli Moehrlen ${ }^{6}$, Rudolf Lucas ${ }^{7 \dagger}$ and Uz Stammberger ${ }^{3,8+}$ \\ ${ }^{1}$ Internal Medicine and Pneumology, Lindenhofspital, Bern, Switzerland, ${ }^{2}$ Internal Medicine V - Pneumology, Allergology, \\ Respiratory and Environmental Medicine, Faculty of Medicine, Saarland University, Saarbrücken, Germany, ${ }^{3}$ Lungen- und \\ Atmungsstiftung Bern, Bern, Switzerland, ${ }^{4}$ Internal Medicine, Sonnenhofspital Bern, Bern, Switzerland, ${ }^{5}$ Faculty of Medicine, \\ Institute of Immunology, University of Regensburg, Regensburg, Germany, ${ }^{6}$ Paediatric Visceral Surgery, Universitäts- \\ Kinderspital Zürich, Zürich, Switzerland, 'Department of Pharmacology and Toxicology, Vascular Biology Center, Medical \\ College of Georgia, Augusta, GA, United States, ${ }^{8}$ Novartis Institutes for Biomedical Research, Translational Clinical \\ Oncology, Novartis Pharma AG, Basel, Switzerland
}

OPEN ACCESS

Edited by:

Niccolo Terrando,

Duke University, United States

Reviewed by:

Gene Thomas Yocum, Columbia University, United States

Michael Wilson,

Imperial College London, United Kingdom

*Correspondence:

Jürg Hamacher

hamacher@greenmail.ch

tThese authors have contributed equally to this work.

Specialty section: This article was submitted to Inflammation, a section of the journal Frontiers in Immunology

Received: 09 August 2017 Accepted: 10 November 2017 Published: 04 January 2018

Citation:

Hamacher J, Hadizamani Y, Borgmann M, Mohaupt M, Männel DN, Moehrlen U, Lucas R and Stammberger $U$ (2018) Cytokine-lon Channel Interactions in Pulmonary Inflammation.

Front. Immunol. 8:1644. doi: 10.3389/fimmu.2017.01644
The lungs conceptually represent a sponge that is interposed in series in the bodies' systemic circulation to take up oxygen and eliminate carbon dioxide. As such, it matches the huge surface areas of the alveolar epithelium to the pulmonary blood capillaries. The lung's constant exposure to the exterior necessitates a competent immune system, as evidenced by the association of clinical immunodeficiencies with pulmonary infections. From the in utero to the postnatal and adult situation, there is an inherent vital need to manage alveolar fluid reabsorption, be it postnatally, or in case of hydrostatic or permeability edema. Whereas a wealth of literature exists on the physiological basis of fluid and solute reabsorption by ion channels and water pores, only sparse knowledge is available so far on pathological situations, such as in microbial infection, acute lung injury or acute respiratory distress syndrome, and in the pulmonary reimplantation response in transplanted lungs. The aim of this review is to discuss alveolar liquid clearance in a selection of lung injury models, thereby especially focusing on cytokines and mediators that modulate ion channels. Inflammation is characterized by complex and probably time-dependent co-signaling, interactions between the involved cell types, as well as by cell demise and barrier dysfunction, which may not uniquely determine a clinical picture. This review, therefore, aims to give integrative thoughts and wants to foster the unraveling of unmet needs in future research.

Keywords: epithelial sodium channel, $\mathrm{Na}^{+} / \mathrm{K}^{+}-$ATPase, tumor necrosis factor, TNF tip peptide, pneumonia, acute respiratory distress syndrome, lung transplantation, ischemia-reperfusion injury

\section{INTRODUCTION}

Acute lung injury (ALI) and acute respiratory distress syndrome (ARDS) are both clinical syndromes with a high morbidity and mortality rate. Although of a different degree of severity, both ARDS and ALI are characterized by critical gas exchange disturbances, an inflammatory reaction, and an associated alveolar fluid overload (edema). The etiology of ALI and ARDS can be differentiated between direct and indirect lung injury. 
The conceptual work presented here discusses the mechanisms regulating alveolar fluid clearance (AFC) during inflammation. As recently demonstrated by several groups, the interaction between cytokines and ion channels may play a critical role in this setting. The presented review does not cover all cytokines and ion channels, but rather focuses on a selection of mainly pre-clinical pathophysiological models and addresses clinical needs and difficulties to effectively translate pre-clinical data into the clinical field. Tables 1-3 give an overview on ion channels and mediator interaction. The ultimate aim of this translational research should be to improve patient care and to reduce morbidity and mortality. This can be achieved by reducing long-term residual sequelae and time on the ventilator, which can improve long-term lung function and health status or health-related quality of life.

The main task of the lungs is to account for the efficient external gas exchange between air and the blood. Only a thin barrier of several micrometers separates the pulmonary capillaries from the immense alveolar surface, mainly made up by alveolar type I cells. An intimately fine, deformable, tensible, flexible, and continuous net of interstitial tissue integrates the interstitial net around vessels and bronchi. The whole system has to be "breathable," i.e., has to be efficiently moved by the thoracic cage to transport fresh air in the alveolar space that matches to the vascular bed for gas exchange. A number of structural and physiological features prevent alveolar flooding. These protective mechanisms include the very low vascular resistance in the pulmonary circulation, the high capillary colloid-osmotic pressure and, on the other hand, the diminished interstitial colloid-osmotic pressure in case of increased filtration. The minimal mechanic stress of alveolar septa due to surface tension reduction by surfactant as well as the optimal active fluid reabsorption out of the alveolar space are further measures that optimize fluid clearance. Structurally, a rather tight pulmonary microvascular endothelium allows for a minimal continuous filtration of water, micro-and macromolecules, with an even tighter alveolar epithelium (1). All three fluid compartments, the capillaries, the interstitium, and the alveoli are in a complex dynamic equilibrium. The continuous pulmonary interstitial space is a drainable continuum that is ultimately emptied by the lymphatic vessels. There is a basal transendothelial filtration of about $10 \mathrm{ml} / \mathrm{h}$ that increases up to tenfold during physical activity. When such filtered fluid enters the alveolar interstitial space, it moves proximally to the peri-bronchovascular space (2). Under normal conditions, most of this filtered fluid will be removed by the lymphatics from the interstitium and returns to the systemic circulation (2).

The interstitial compartment is a reversible store of excess fluid. In the adult lung, interstitial fluid — or interstitial edemacan mount up to a volume of $500 \mathrm{ml}$. However, at that volume there is usually already some alveolar edema (3). It was formerly wrongfully postulated that the Starling filtration forces, which essentially represent the balance between oncotic and hydrostatic pressures in the capillaries and the interstitial space, are the only driving forces for liquid flow from the bloodstream into the extravascular space. In the last four decades, four important refinements have been made. The first one is that fluid reabsorption from the alveolar space is mainly performed by active vectorial $\mathrm{Na}^{+}$transport (4). Moreover, also $\mathrm{Cl}^{-}$transport was suggested to be important, leading to consecutive counter-ion transport, as well as to an osmotic water shift. In the last few years, a second refinement has been made which mainly occurs in heart failure, namely that pumps which usually free the alveolus of ions can also provide inverse transport (5). This biological "emergency plan" in case of hydrostatic pulmonary edema widens the scope of mechanisms in cardiogenic lung edema, as one can argue that in heart failure these mechanisms could be rescue fluid shifts including into the alveolar space, and that a concerted fluid management in vascular, renal, and intestinal and pulmonary vascular beds might occur in severe cardiac failure or fluid overload, taking into account some degree of alveolar pulmonary edema. A third rather novel field is the research on emptying of the alveolar space from its protein load; but so far only few insights in this clinical topic exist (6). The fourth refinement is the close relationship of ion channel activation with barrier tightness. Interactions between the lectin-like domain of tumor necrosis factor (TNF), mimicked by its amino acid-identic TNF tip peptide (a.k.a. AP301 and Solnatide) and the epithelial sodium channel $(\mathrm{ENaC})$ were shown to have a clear effect on epithelial (7) and endothelial barrier tightness $(8,9)$. As such, ion channel activity and barrier tightness may be key survival factors for tissue function, be it the lung or the kidney, the brain or other organs, and for tissue stability (9-11).

Alveolar fluid reabsorption is a very physiological process that is even required directly after birth where the lung has to be cleared from liquid as it has been so far immersed in the amnionic fluid. In premature infant, insufficient clearance of lung liquid at birth may lead to respiratory distress syndrome (RDS). The key clinical relevance of the physiological role of $\alpha \mathrm{ENaC}$ in the lungs has been confirmed in the mouse in which the $\mathrm{ENaC}-\alpha$ gene was deleted by a homologous recombination. These animals were not able to remove alveolar fluid from their lungs and died shortly after birth (12). Surprisingly, in humans this situation seems more complex, as a child with an inactive homozygous ENaC- $\alpha$ mutation did not suffer perinatal respiratory failure (13).

Likewise in adults with heart failure or RDS, while they show no active fluid clearance greater morbidity and mortality rate is probable (14). In clinical studies using quantification of protein in alveolar liquid, prognosis was dependent on the estimated AFC. In a recent study, 56\% had impaired AFC, and only $13 \%$ a maximal AFC rate (Figure 1). Survival was higher and days on mechanical ventilation were less in those patients with maximal alveolar clearance rate compared to patients with impaired clearance rate. With hydrostatic edema, by contrast, $75 \%$ of patients had submaximal to maximal AFC (15). Of note is that in hydrostatic edema alveolar fluid shift may even actively be reversed $(5,16)$ as discussed above.

\section{PULMONARY EDEMA}

Pulmonary alveolar edema is a life-threatening state that results from an imbalance between passive and active forces driving fluid into the airspaces and those mechanisms involved in its removal $(1,4)$. Based on the underlying cause, in the next two chapters we will discuss two main fundamentally different types of pulmonary edema occur in humans (2). 
TABLE 1 | Role of different mediators on fluid transport through impacting on ion channels in the apical and basolateral membrane of epithelial cells.

Channel name Mediator $\begin{gathered}\text { Impact on } \\ \text { pulmonary } \\ \text { barrier function }\end{gathered}$

Apical membrane

Epithelial sodium $\quad$ Transforming growth factor $\quad-/+\quad$ Decrease in expression during bacterial infection (132)

channel $(\mathrm{ENaC}) \quad$ beta $(\mathrm{TGF}-\beta) \quad$ Decreases expression of the $\alpha \mathrm{ENaC} \mathrm{mRNA}$ and protein (132)

Internalization of $\alpha \beta \gamma E N a C$ complex from the lung epithelial cell surface and, hence, block the sodium-transporting capacity of alveolar epithelial cells (AECs) (133) Increases the function of ENaC (134)

\begin{tabular}{lll}
\hline $\begin{array}{l}\text { Tumor necrosis factor (TNF) } \\
\text { receptor binding site }\end{array}$ & - & Decreases the expression of ENaC mRNA in AECs in vitro (135) \\
\hline TNF lectin-like domain & + & $\begin{array}{l}\text { Activates ENaC (37, 136) } \\
\text { Increases ENaC open probability (102) }\end{array}$ \\
\hline
\end{tabular}

Interleukin-1 $\beta(\mathrm{IL}-1 \beta) \quad$-/+ $\quad$ Decreases the expression of ENaC during bacterial infection (113)

Decreases expression of $\alpha \mathrm{ENaC}$ via a p38 MAPK-dependent signaling pathway (113)

Suppresses expression of $\beta \mathrm{ENaC}$ (137)

Decreases ENaC function (138)

Augments in vitro alveolar epithelial repair (139)

Increases ENaC subunits expression in a specific fetal context (140)

Interleukin-4 (IL-4) _ _ _ _ Decreases in ENaC expression during bacterial infection (141)

Decreases ENaC activity by decreasing the mRNA levels of $\gamma \mathrm{ENaC}$ and, to a lesser extent, that of the $\beta$ subunit (142)

\begin{tabular}{lll}
\hline $\begin{array}{l}\text { Keratinocyte growth factor } \\
\text { (FGF-7) }\end{array}$ & - & Decreases the expression of $\alpha E N a C$ (143) \\
\hline Protein kinase C (PKC) & - & Inhibits ENaC function (144-147) \\
\hline
\end{tabular}

Cycloheximide $(\mathrm{CHX}) \quad$ - $\quad$ Downregulate $\alpha$ ENaC mRNA abundance similarly via the ERK and p38 MAPK pathway (148); Chx effect involves post-transcriptional mechanisms (148)

Lipopolysaccharide (LPS) _ _ _ _ Downregulates $\alpha$ ENaC mRNA abundance similarly via the ERK and p38 MAPK pathways

(148); inhibits $\alpha E N a C$ promoter activity (148)

Pneumolysin (PLY) _ _ _ Inhibits ENaC expression upon activation of ERK (102) and inhibits ENaC open probability, by

reducing its association with myristoylated alanine-rich $\mathrm{C}$ kinase substrate (10, 149)

Glutathione disulfide (GSSG) _ _ _ Inhibits ENaC activity in primary AECs (150, 151)

\begin{tabular}{|c|c|c|}
\hline $\begin{array}{l}\text { Reactive oxygen species } \\
\text { (ROS) }\end{array}$ & $-/+$ & $\begin{array}{l}\text { Inhibit ENaC }(150,152) \\
\text { Decrease channel activity (117) } \\
\text { Increases ENaC activity through: }\end{array}$ \\
\hline
\end{tabular}

Increases ENaC activity through:

(i) Enhancing ENaC gating (153)

(ii) Increasing channel abundance (153)

\begin{tabular}{|c|c|c|}
\hline Ethanol & + & $\begin{array}{l}\text { Increases ENaC open-state probability (153) } \\
\text { Increases ENaC abundance (153) }\end{array}$ \\
\hline Superoxide $\left(\mathrm{O} 2^{\mathrm{a}}\right)$ & + & $\begin{array}{l}\text { Elevating endogenous }\left(\mathrm{O}_{2}^{-}\right) \text {levels with a superoxide dismutase inhibitor, prevents } \\
\mathrm{NO} \text { inhibition of ENaC activity (111) }\end{array}$ \\
\hline Nitric oxide (NO) & - & Inhibits highly selective sodium channels $(52,53)$ \\
\hline Inter- $\alpha$-inhibitor $(|\alpha|)$ & - & Inhibits ENaC activity in CF patients (154) \\
\hline NEDD4-2 & - & Decreases the expression of the epithelial ENaC (155) \\
\hline Hypoxia & - & Decreases apical expression of ENaC subunits (especially beta and gamma) (156) \\
\hline Purinergic receptors (P2YR) & - & Inhibits ENaC expression $(157,158)$ \\
\hline Muscarinic cholinergic & + & Increases ENaC activity. RhoA activity is essential for this process (159) \\
\hline Estriadol & + & $\begin{array}{l}\text { Increases activity of the non-selective ENaC channels, and these effects are mediated } \\
\text { through the G protein-coupled estrogen receptor (160) }\end{array}$ \\
\hline Glucocorticoids & + & Increased in expression of ENaC during bacterial infection (161-164) \\
\hline Thyroid hormone & + & $\begin{array}{l}\text { Thyroid hormone in concert with glucocorticoids increased the expression of } \\
\text { ENaC }(165,166)\end{array}$ \\
\hline Corticosteroids & + & Increase expression of the $\gamma$-ENaC subunit which leads to increase ENaC activity (167) \\
\hline
\end{tabular}

(Continued) 
TABLE 1 | Continued

Channel name

\section{Mediator}

\section{Impact on \\ pulmonary \\ barrier function \\ Mechanism of action}

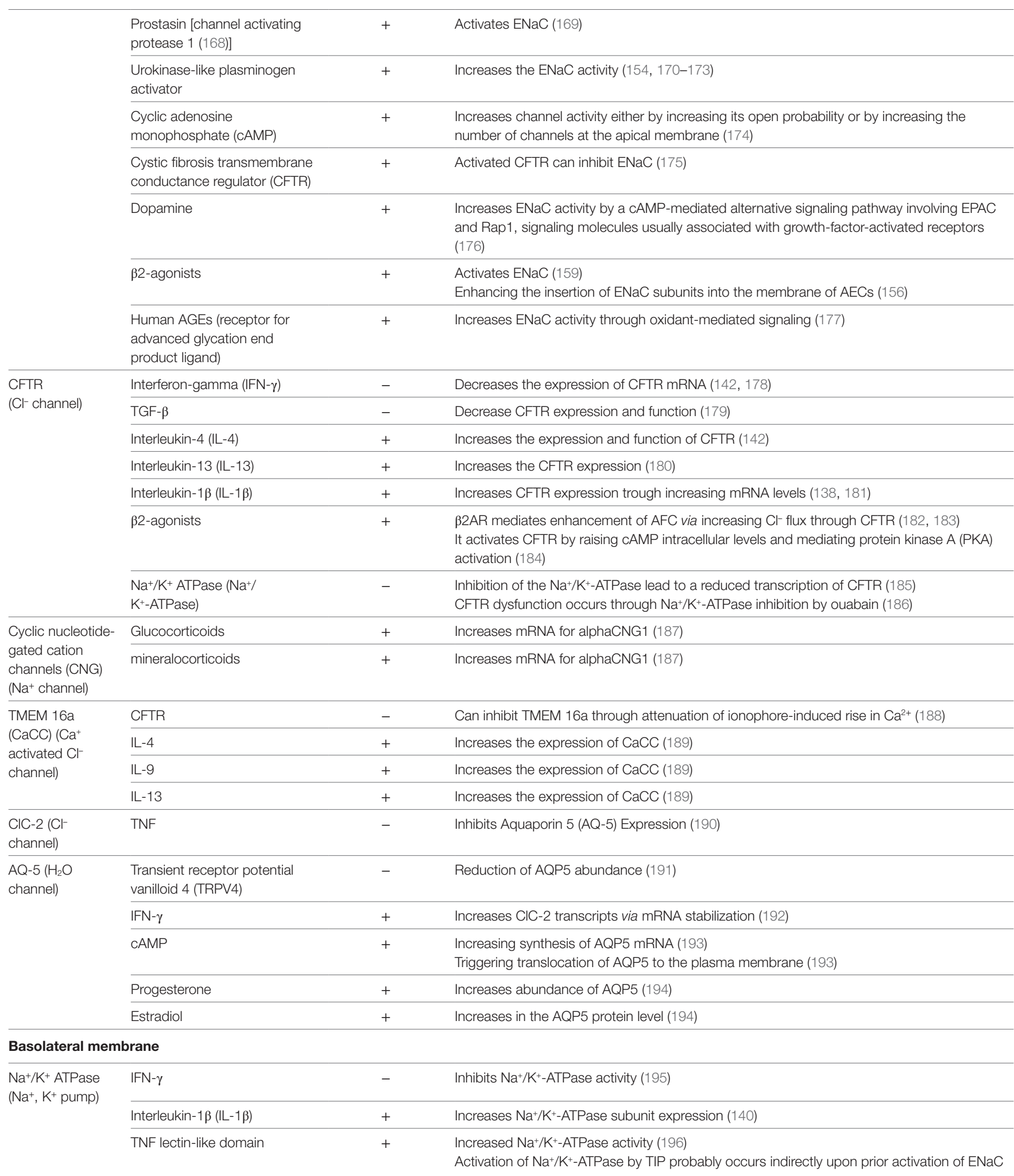

n 
TABLE 1 | Continued

\begin{tabular}{|c|c|c|c|}
\hline Channel name & Mediator & $\begin{array}{l}\text { Impact on } \\
\text { pulmonary } \\
\text { barrier function }\end{array}$ & Mechanism of action \\
\hline & TGF- $\beta$ & $-/+$ & $\begin{array}{l}\text { Decrease in } \mathrm{Na}^{+} / \mathrm{K}^{+} \text {-ATPase } \beta 1 \text { subunit expression, resulting in decreased } \mathrm{Na}^{+} / \mathrm{K}^{+} \text {-ATPase } \\
\text { activity }(197,198) \\
\text { Increases the expression of } \mathrm{Na}^{+} / \mathrm{K}^{+} \text {-ATPase } \alpha 1 \text { - and } \beta 1 \text {-subunits (134) }\end{array}$ \\
\hline & $\begin{array}{l}\text { TNF-related apoptosis- } \\
\text { inducing ligand (TRAIL) }\end{array}$ & - & $\begin{array}{l}\text { Influenza A virus (IAV)-induced reduction of } \mathrm{Na}^{+} / \mathrm{K}^{+}-\mathrm{ATP} \text { ase is mediated by a host signaling } \\
\text { pathway that involves epithelial type I IFN and an IFN-dependent elevation of macrophage } \\
\text { TRAIL (199) }\end{array}$ \\
\hline & Leukotriene D4 & + & Activates $\mathrm{Na}^{+} / \mathrm{K}^{+}$-ATPase (200) \\
\hline & Acetylcholine & + & Activates $\mathrm{Na}^{+} / \mathrm{K}^{+}$-ATPase (201) \\
\hline & NO & - & Inhibits $\mathrm{Na}^{+} / \mathrm{K}^{+}$-ATPase $(53,202)$ \\
\hline
\end{tabular}

aThere is growing evidence that ROS are important regulators of ENaC activity and, hence, of epithelial Na+ absorption (153). But there is an important question here. Why does ROS increase ENaC activity under some circumstances (e.g., ethanol) but inhibit ENaC under others (e.g., influenza) (153)?

\section{Cardiogenic or Hydrostatic Edema}

Cardiogenic pulmonary edema (also called hydrostatic or hemodynamic edema) (2) is caused by an increased capillary hydrostatic pressure, secondary to an elevated pulmonary venous pressure (18) (Figure 2, left panel). This type of edema can occur following left ventricular heart failure, renal failure, or fluid overload, or arteriovenous shunts or fistulas. Left heart failure is most commonly caused by myocardial ischemia with or without myocardial infarction, exacerbation of chronic systolic or diastolic heart failure, or dysfunction of the mitral or aortic valve. Acute cardiogenic pulmonary edema is a frequent medical emergency that accounts for up to 1 million hospital admissions per year in the United States and for about 6.5 million hospital days each year, and is typically present during acute cardiac failure in $75-80 \%$ of patients (19). Coronary heart disease may account for about half to two-thirds of heart failures. There has been an increase in cardiac failure patients as well as in hospitalization rate during the last decade (20). As a matter of fact, heart failure is the most rapidly growing cardiovascular condition globally. The reported Western world life time risk is typically about $33 \%$ for men and $29 \%$ for women for our population, and depends, besides sex, on comorbidities and cardiovascular risk factors, such as arterial hypertension, diabetes, obesity, sleep related disorders, smoking, sedentary lifestyle, and ethnic background (20). In patients aged 65 years and older, more than $10 \%$ suffer from congestive heart failure (21). Interstitial pulmonary edema and alveolar flooding impair lung mechanics and gas exchange, thus causing dyspnea and tachypnea, which ultimately results in an age-dependent inhospital mortality rate of about 15\% (22).

The development of pulmonary edema is characterized by increased transcapillary hydrostatic pressure gradients. Moreover, a reversed and active electrolyte flow and its resulting active fluid transport can be involved $(5,23)$. This is possible by the bidirectional permeation permitting anion channels cystic fibrosis transmembrane conductance regulator (CFTR) and NKCC1 (16), which seems to account for up to $70 \%$ of the total alveolar fluid influx at elevated hydrostatic pressure. It is supporting the concept that alveolar fluid secretion is a secondary consequence of impaired alveolar $\mathrm{Na}^{+}$uptake (16). Both CFTR and NKCC1 are inhibited by furosemide. This might explain why in the clinical heart failure setting furosemide immediately relieves patients, i.e., by inhibition ion and, thus, fluid transport into the alveolus during alveolar lung edema generation when furosemide is administered, and not only after a huger delay of about half an hour or more when the renal effect of relevant diuresis has occurred. However, also a venous vasodilation, direcly reducing preload, occurs immediately after systemic furosemide administration (24).

A rapid increase in hydrostatic pressure in the pulmonary capillaries, leading to increased transvascular fluid filtration, and even active fluid transport as mentioned above, is the sign of acute cardiogenic or volume-overload edema (Figure 2, left panel). Such an increase could be usually due to elevated pulmonary venous pressure from increased left ventricular end-diastolic pressure and left atrial pressure (2). Mild elevations of left atrial pressure $(18-25 \mathrm{mmHg}$ ) cause edema in the perimicrovascular and peri-bronchovascular interstitial spaces (1). Excess interstitial fluid is transported by lung lymphatics into the vascular system. A negative interstitial pressure gradient, even under conditions of edema, is the major force for the removal of pulmonary interstitial edema fluid into the lymphatics (25). If left atrial pressure rises further $(>25 \mathrm{mmHg}$ ), edema fluid passes through the lung epithelium, in part by active transport, flooding the alveolar space with protein-poor fluid (Figure 2, left panel) $(1,2,5)$. By contrast, non-cardiogenic pulmonary edema is based on increased pulmonary vascular permeability, resulting in an increased flux of fluid and macromolecules into the pulmonary interstitium and airspaces (Figure 2, right panel) (2).

There is a considerable link between inflammation and heart failure. The Val-HeFT study demonstrated a direct correlation between elevated levels of C-reactive protein and heart failure severity, and C-reactive protein predicts the risk of death and early readmission in acutely decompensated heart failure (26). As reviewed by Azzam et al. in this topic issue, one hypothesis is that heart failure is accompanied by systemic and mesenteric venous congestion, which may in turn cause bowel edema and a consecutive increased permeability, leading to bacterial translocation, endotoxin release, and resultant systemic inflammation. A second hypothesis postulates that the failing, but not the healthy, 
TABLE 2 | Impact of different factors on the alveolar-capillary barrier.

\begin{tabular}{ll}
\hline Mediator & $\begin{array}{c}\text { Impact on } \\
\text { pulmonary } \\
\text { barrier function }\end{array}$
\end{tabular}

\section{Alveolar epithelium}

\begin{tabular}{|c|c|c|}
\hline TGF $\beta 1$ & - & $\begin{array}{l}\text { Decreases lung epithelial barrier function (203-205) } \\
\text { Increases the permeability of pulmonary endothelial monolayers (206) } \\
\text { Increases the permeability of alveolar epithelial monolayers (206) }\end{array}$ \\
\hline Tumor necrosis factor (TNF) & - & Causes alveolar epithelial dysfunction (207) \\
\hline Lectin-like domain of TNF & + & Increases occludin expression, and improved gas-blood barrier function (7) \\
\hline $\begin{array}{l}\text { TNF-related apoptosis-inducing } \\
\text { ligand (TRAIL) }\end{array}$ & - & Disruption of alveolar epithelial barrier $(199,208,209)$ \\
\hline Interleukin-1 $\beta(\mathrm{IL}-1 \beta)$ & + & Augments in vitro alveolar epithelial repair (139) \\
\hline Protein kinase D3 & - & Dysfunction of airway epithelial barrier through downregulation of a key tight junctional protein claudin-1 (210) \\
\hline Claudin-3 & - & Decreases alveolar epithelial barrier function (211) \\
\hline Claudin-4 & + & $\begin{array}{l}\text { Improves the barrier function of pulmonary epithelial barrier by promoting pulmonary fluid-clearance function } \\
(211,212)\end{array}$ \\
\hline $\begin{array}{l}\text { Transient receptor potential } \\
\text { vanilloid } 4 \text { (TRPV4) }\end{array}$ & - & Disruption of alveolar type I epithelial cells leading to lung vascular leak and alveolar edema (213) \\
\hline Ethanol & - & Disruption of alveolar epithelial barrier function by activation of macrophage-derived TGF $\beta 1$ (214) \\
\hline
\end{tabular}

Acetoin (butter), diacetyl, pentanedione, maltol (malt), ortho-vanillin (vanilla), coumarin, and cinnamaldehyde

\begin{tabular}{|c|c|c|}
\hline Asbestos & - & Increases lung epithelial permeability through increasing epithelial fibrinolytic activity (216) \\
\hline Pneumolysin (PLY) & - & Impairs epithelial barrier (217) \\
\hline Fas-ligand system & - & $\begin{array}{l}\text { Causes alveolar epithelial injury in humans with ALI or ARDS (218) } \\
\text { Impairs alveolar epithelial function in mouse lungs by mechanisms involving caspase-dependent apoptosis (219) } \\
\text { Inducing apoptosis of cells of the distal pulmonary epithelium during ALI (57) }\end{array}$ \\
\hline $\mathrm{CO}$ & - & Enhances pulmonary epithelial permeability $(220,221)$ \\
\hline
\end{tabular}

\section{Tight junctions (TJ)}

\begin{tabular}{|c|c|c|}
\hline Purinergic receptor & + & Preserving integrity of endothelial cell (EC)-cell junctions (222) \\
\hline \multirow[t]{2}{*}{$\mathrm{Na}^{+} / \mathrm{K}^{+}$ATPase } & + & Formation of TJs through RhoA GTPase and stress fibers (223) \\
\hline & + & $\begin{array}{l}\text { Gene transfer of } \beta 1-\mathrm{Na}^{+}, \mathrm{K}^{+}-\text {ATPase upregulates TJs formation by enhancing expression of TJ protein zona } \\
\text { occludins- } 1 \text { and occludin and reducing pre-existing increase of lung permeability (224) }\end{array}$ \\
\hline Nitric oxide (NO) & - & Decreases expression and mistargeting of TJ proteins in lung (225) \\
\hline Influenza A virus (IAV) & - & Disruption epithelial cell TJs (226) \\
\hline Caveolin-1 & + & Regulates the expression of TJ proteins during hyperoxia-induced pulmonary epithelial barrier breakdown (227) \\
\hline \multirow[t]{2}{*}{ IL-4 } & - & $\begin{array}{l}\text { Causes TJ disassembly and epithelial barrier permeability alteration via an EGFR-dependent MAPK/ERK1/2- } \\
\text { pathway (228) }\end{array}$ \\
\hline & & $\begin{array}{l}\text { Reduce protein density at the TJ without causing major changes in cldn1, cldn2, cldn3, and occludin protein } \\
\text { levels (229) }\end{array}$ \\
\hline IL-13 & - & $\begin{array}{l}\text { Reduction of protein density at the TJ without causing major changes in cldn1, cldn2, cldn3, and occludin } \\
\text { protein levels (229) }\end{array}$ \\
\hline TNF & - & Causes TJ permeability (230) \\
\hline \multirow[t]{3}{*}{ Interferon-gamma (IFN- $\gamma)$} & $-/+$ & Disorganization of the TJ and an increase in paracellular permeability (231) \\
\hline & & Promotes epithelial restitution by enhancing barrier function and wound healing (232) \\
\hline & & It can also reverse IL-4- and IL-13-induced barrier disruption (232) \\
\hline Trypsin & - & Destroys the TJs which lead to airway leakage \\
\hline Cigaret smoke & - & Causes disassembly of TJs, modulated through the EGFR-ERK1/2 signaling pathway (233) \\
\hline Cadmium & - & $\begin{array}{l}\text { Causes disruption of TJ integrity in human ALI airway cultures both through occludin hyperphosphorylation } \\
\text { via kinase activation and by direct disruption of the junction-interacting complex (234) }\end{array}$ \\
\hline
\end{tabular}


TABLE 2 | Continued

\begin{tabular}{|c|c|c|}
\hline Mediator & $\begin{array}{l}\text { Impact on } \\
\text { pulmonary } \\
\text { barrier function }\end{array}$ & Mechanism of action \\
\hline \multicolumn{3}{|l|}{ Capillary endothelium } \\
\hline TGF $\beta 1$ & - & Induces endothelial barrier dysfunction via Smad2-dependent p38 activation (235) \\
\hline TNF & - & $\begin{array}{l}\text { Disruption of the lung vascular barrier }(236,237) \\
\text { Augmenting endothelial permeability }(67,238) \\
\text { Apoptosis of lung microvascular ECs }(39,239,240)\end{array}$ \\
\hline Lectin-like domain of TNF & + & $\begin{array}{l}\text { Strengthens barrier function or increasing endothelial barrier tightness (9) } \\
\text { Protective effect in PLY-Induced endothelial barrier dysfunction (9) } \\
\text { Can reduce PLY-induced RhoA/Rac-1 balance impairment and MLC phosphorylation (10) } \\
\text { Protects from listeriolysin-induced hyperpermeability in human pulmonary microvascular ECs (241) } \\
\text { Reducing vascular permeability (196) } \\
\text { Increases in membrane conductance in primary lung microvascular ECs (242) }\end{array}$ \\
\hline $\mathrm{IFN}-\gamma$ & - & Increases vascular permeability (243) \\
\hline Interleukin-1 $\beta(\mathrm{IL}-1 \beta)$ & - & $\begin{array}{l}\text { Given intratracheally, IL-1 } \beta \text { increased endothelial permeability and lung leak (244-247) } \\
\text { Increases vascular permeability (243) }\end{array}$ \\
\hline Interleukin-2 (IL-2) & - & Increases vascular permeability (248) \\
\hline Interleukin-6 (IL-6) & - & Increases endothelial permeability (249) \\
\hline Interleukin-8 (IL-8) & - & Increases endothelial permeability (250) \\
\hline Interleukin -12 (IL-12) & - & $\begin{array}{l}\text { Upregulate the release of the vascular permeability factor which is a lymphokine derived from LN peripheral } \\
\text { blood mononuclear cells (251) }\end{array}$ \\
\hline Neutrophils & - & $\begin{array}{l}\text { Inducing endothelial barrier disruption through secretion of leukotrienes or heparin-binding protein, direct } \\
\text { signaling into the EC via adhesion-dependent mechanisms and production of ROS (252) }\end{array}$ \\
\hline $\mathrm{ENaC}$ & + & ENaC- $\alpha$ can strengthen capillary barrier function (9) \\
\hline TRPV4 & - & $\begin{array}{l}\text { Increases in vascular permeability thus promoting protein and fluid leak (253) } \\
\text { Applying TRPV4 inhibitors exhibits vasculoprotective effects, inhibiting vascular leakage, and improving blood } \\
\text { oxygenation (254) }\end{array}$ \\
\hline Thrombin & - & Increase in endothelial permeability (255) \\
\hline Platelet-activating factor & - & Increase in endothelial permeability (256) \\
\hline Hydrogen peroxide & - & Increase vascular permeability through enhancing vascular endothelial growth factor expression (257) \\
\hline Integrin $\alpha \vee \beta 5$ & - & Increases pulmonary vascular permeability (258) \\
\hline T-cadherin & - & Causes enhancement of endothelial permeability (259) \\
\hline Myosin light chain kinase & - & Vascular hyperpermeability (260) \\
\hline Lipopolysaccharide (LPS) & - & Induces lung endothelial barrier dysfunction (261) \\
\hline PLY & - & Impairs endothelial barrier $(10,262)$ \\
\hline P2Y receptors & + & Regulators of lung endothelial barrier integrity (263) \\
\hline $\mathrm{CO}$ & - & Enhances pulmonary epithelial permeability (221) \\
\hline $\begin{array}{l}\text { Soluble receptor for advanced } \\
\text { glycation end products }\end{array}$ & - & Increase in alveolar-capillary barrier permeability (264) \\
\hline
\end{tabular}

\section{EC adhesion}

\begin{tabular}{lll}
\hline Podocalyxin & + & Decreases vascular permeability of ECs by altering EC adhesion (265) \\
\hline NLRP3 & + & Protects alveolar barrier integrity by an inflammasome-independent increase of epithelial cell adherence (266)
\end{tabular}

heart has the ability to produce pro-inflammatory TNF during dilated myopathy. Third, decreased cardiac output could cause systemic tissue hypoxia with subsequent systemic inflammation, which might be the primary stimulus for increased TNF production (21).

Soluble TNF receptor-1 and interleukin-8 (IL-8) are independently associated with cardiovascular mortality, as is endothelin-1. In transgenic mice overexpressing TNF the left ventricular ejection fraction was depressed depending on TNF gene dosage (21). TNF has been associated with worsened prognosis. However, two studies aiming to neutralize the cytokine in heart failure, using the soluble human TNF receptor 2 construct etanercept, were stopped because of lack of clinical benefit and patients receiving the highest dose even had increased adverse 
TABLE 3 | Comparison of the properties of highly selective and non-selective channels.

\begin{tabular}{|c|c|c|}
\hline & Highly Selective & Non-selective \\
\hline $\mathrm{Na} / \mathrm{K}$ selectivity $(267,268)$ & $>40$ & 1.1 \\
\hline Unit conductance, pS (267-269) & 6 & 21 \\
\hline Amiloride $\mathrm{K}_{\mathrm{i}}, \mathrm{nM}(268,270)$ & 38 & 2,300 \\
\hline $\begin{array}{l}\text { Increased cellular cyclic adenosine } \\
\text { monophosphate or } \beta \text {-adrenergic } \\
\text { stimulation }(271,272)\end{array}$ & $\begin{array}{l}\text { Channel surface } \\
\text { density increases }\end{array}$ & Po increases \\
\hline Increased cGMP or NO (273) & Po decreases & $P_{\circ}$ decreases \\
\hline Protein kinase $\mathrm{C}$ activation $(274,275)$ & $\begin{array}{l}\text { Po decreases, } \\
\text { surface density } \\
\text { decreases }\end{array}$ & $\begin{array}{l}\text { Channel surface } \\
\text { density increases }\end{array}$ \\
\hline Increased intracellular $\mathrm{Ca}^{2+}(271)$ & No effect & Po increases \\
\hline Purinergic stimulation (276-279) & Po decreases & Po increases \\
\hline Dopaminergic stimulation $(176,280)$ & $P_{\circ}$ increases & No effect \\
\hline Superoxide production (111) & $P_{\circ}$ increases & $\begin{array}{l}\text { Channel surface } \\
\text { density increases }\end{array}$ \\
\hline Hypoxia (268) & $\begin{array}{l}\text { Channel surface } \\
\text { density decreases }\end{array}$ & $\begin{array}{l}\text { Channel surface } \\
\text { density increases }\end{array}$ \\
\hline
\end{tabular}

$P_{\text {o }}$, channel open probability; $K_{i}$ inhibitory constant, i.e., the dose that reduces open probability by $50 \%$.



FIGURE 1 | Hospital mortality is increased in patients with acute lung injury or the acute respiratory distress syndrome with impaired fluid clearance (17).

outcomes (27). Similar results were observed with the neutralizing antibody infliximab (28). Whether the negative results are explained by inappropriate blocking of a "physiological" inflammation linked with tissue-reparative processes such as cardiac remodeling, or whether other mechanisms like too advanced heart failure, infections, toxicity of treatment, or genetic polymorphisms are involved, remains open, and should be further studied (21). Recently, it was suggested that beneficial or detrimental effects of TNF neutralizing agents depend on whether they spared or rather blunted discrete amounts of TNF that preconditioned cardiomyocytes to make them more resistant to high concentrations of the cytokine (29). The results, however, put forward that cytokines are effectors and not solely biomarkers in heart failure. Furthermore, reparative processes in the myocardium are accompanied by reactive or replacement fibrosis, mediated by TGF- $\beta 1$, endothelin-1, and angiotensinII (21). Angiotensin-II decreases AFC via cyclic adenosine monophosphate (cAMP) effect on the $\mathrm{Na}^{+} / \mathrm{K}^{+}$-ATPase pathway.
It is involved through p38 and possibly p42/44 MAP kinases with myocardial hypertrophy, inflammation, and neurotransmitter and catecholamine synthesis and release in the brain. Angiotensin-II regulates the NF- $\mathrm{kB}$-dependent gene expression in response to IL- $1 \beta$ stimulation by controlling the duration of ERK and NF- $\kappa B$ activation (21). Many immune cell functions are moreover coupled to intracellular $\mathrm{pH}$. As such, a higher $\mathrm{pH}$ represents an important signal for cytokine and chemokine release, and a low $\mathrm{pH}$ can induce an efficient antigen presentation. The $\mathrm{pH}$ regulating $\mathrm{Na}^{+} / \mathrm{H}^{+}$exchanger isoforms may play a role in these events (30).

The kidney is a major target organ and a modulator in the pathogenesis of heart failure at least partially by means of the renin-angiotensin system. In initial heart failure, it aims at blood pressure maintenance by direct systemic vasoconstriction, via augmentation of the sympathetic nervous system activity and by promoting renal $\mathrm{Na}^{+}$retention. The latter mechanism is deleterious in the progress of cardiac failure and is characterized by enhanced $\mathrm{Na}^{+}$reabsorption in the proximal tubule and collecting duct induced by effects of angiotensin-II and aldosterone on NHE3 and ENaC, respectively (21). Two-thirds of filtered $\mathrm{Na}^{+}$ is reabsorbed in the proximal tubule via transporters for amino acids, glucose, phosphate and via NHE3. At the distal tubule, $\mathrm{Na}^{+}$is reabsorbed by $\mathrm{Na}^{+}, \mathrm{K}^{+}$co-transporter, which is sensitive to thiazide. In the collecting ducts, a minimal amount of sodium is reabsorbed by $\mathrm{ENaC}$ and this is increased by aldosterone. The counterbalance by the natriuretic and vasodilatory atrial natriuretic peptide is dominated at that point by angiotensin-II and aldosterone effects, attenuates endothelial-dependent renal vasodilation and leads to endothelial dysfunction characteristic of cardiac heart failure (21). Heart failure also causes a vasopressindependent water reabsorption which maintains blood pressure in the failing heart and further increases fluid retention. The renin-angiotensin system, especially angiotensin-II, activates the immune system and vice versa. TNF and IL-6 stimulate the generation of angiotensinogen, exaggerate sodium retention and enhance renal fibrosis. Angiotensin-II enhances TNF and IL-6 in cardiomyocytes and in renal cortical and tubular cells, impairs mitochondrial function, and is pro-oxidative (21). CRP also directly activates endothelin and by this may potentiate a pulmonary vasoconstriction. The review by Azzam et al. in this issue further discusses the causative role of cytokines in the development of cardiogenic edema.

\section{Non-Cardiogenic or Permeability Pulmonary Edema}

Non-cardiogenic pulmonary edema, also known as permeability pulmonary edema, accompanies ALI, pneumonia, pulmonary reimplantation response after lung transplantation, or ARDS $(2,31)$ (Figure 2, right panel). During the course of these diseases, the interstitium and the alevolus are sites of intense inflammation by an innate immune cell-mediated damage of the alveolar endothelial and alveolar epithelial barrier, with consecutive exudation of protein-rich pulmonary edema fluid (31-33), as recently reviewed by Thompson et al. (31). 


\section{Cardiogenic pulmonary oedema}


\section{Noncardiogenic permeability-type pulmonary oedema}

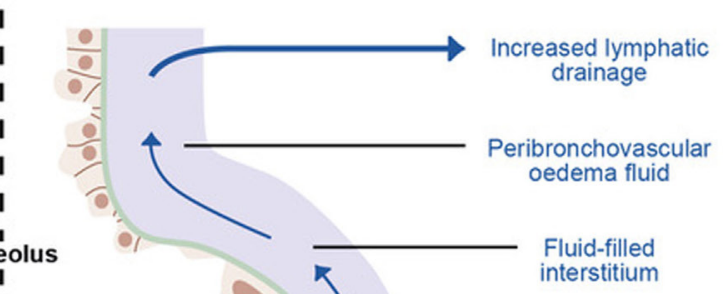

olus

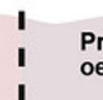

Protein-rich

Impaired

transport of $\mathrm{Na}^{+}$

$\mathrm{Cl}^{*}$, and $\mathrm{H}_{2} \mathrm{O}$ with

I $\mathrm{Cl}^{\circ}$, and $\mathrm{H}_{2} \mathrm{O}$ with
I reduced clearance
of oedema fluid

I $\mathrm{Cl}^{\circ}$, and $\mathrm{H}_{2} \mathrm{O}$ with
I reduced clearance
of oedema fluid

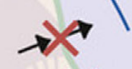

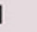

I

I

Macrophage

Aveolar flooding due

Normal

hydrostatic

pressure

(Pmv) oedema fluid

I to increased epith

I

$\uparrow \bigcirc$
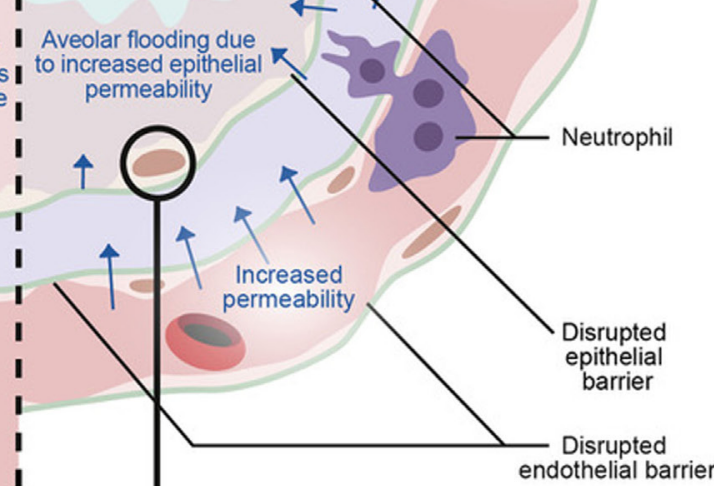

I 
This type of pulmonary edema occurs due to modifications in barrier function of the pulmonary capillary or alveolar epithelial compartments as a consequence of either a direct or an indirect pathological process (31). There is some evidence that direct injury, such as pneumonia, aspiration, or pulmonary contusion, mainly affects epithelial barriers, whereas indirect blood-borne insults such as severe sepsis, non-thoracic trauma, pancreatitis, or burns may predominantly target the capillary endothelium (34). Permeability edema accompanies a spectrum of illnesses, ranging from the less severe form of ALI to ARDS (18). Variations in histology and in fluid management strategies suggest different ARDS subphenotypes (31). Apart from ARDS, ALI and severe pneumonia, also lung transplantation can be accompanied by acute pulmonary edema by the pulmonary reimplantation response (35). Ischemic vascular injury of the allograft results in increased permeability of the lung after reperfusion and in turn leads to interstitial and alveolar edema (33).

The extent of alveolar edema depends on the competing effects of increased permeability and the active edema fluid clearance from the alveolar space in regions where the epithelium is undamaged $(31,36)$. Inflammation plays a key role in the pathogenesis of permeability edema $(37,38)$ and can lead to the orchestration of a great variety of inflammatory and non-inflammatory cells, the former of which can locally release pro-inflammatory mediators such as TNF, LTD4 (32). There may also be endothelial and alveolar epithelial cell (AECs) death, which can further contribute to organ dysfunction and leak $(39,40)$. Moreover, a cascade of inflammation and a downregulation of repair mechanisms may occur (Figures 3, 4).

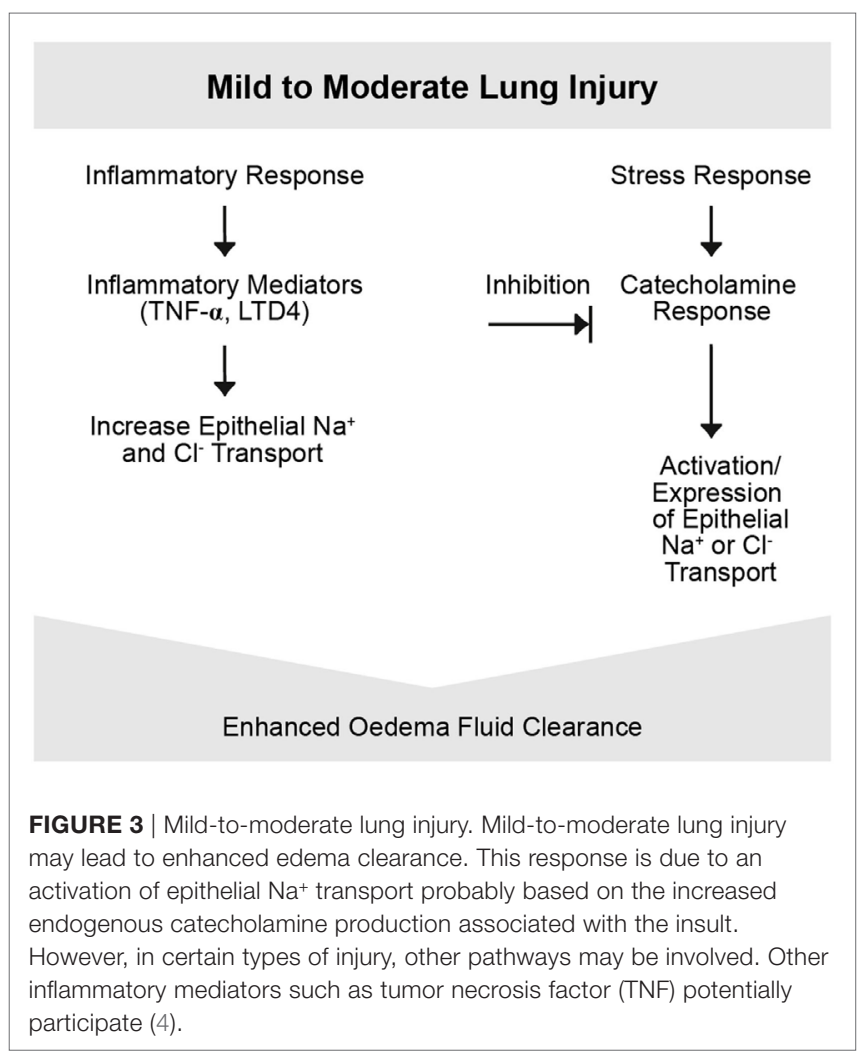

Cells of the innate immune system, such as activated alveolar macrophages and recruited polymorphonuclear granulocytes (PMN) and also cells from the adaptive immune system, such as $\mathrm{T}_{\mathrm{H}} 17$ cells can interact in ALI and ARDS and release huge amounts of mediators (31). Thrombo-coagulative processes ensue, e.g., TNF-mediated by tissue factor, with a proaggregatory role for platelets. Preventive aspirin was recently shown to protect from ARDS (41). Regional tissue overdistension especially during ventilation and repetitive opening and closing of inflamed alveolar spaces amplify the regional inflammation, further denaturing surfactant, underlining the vital importance of protective ventilation strategies and positions.

Although pulmonary edema is one of the most frequent medical emergencies, clinically it is sometimes difficult to differentiate between its two main subtypes: cardiogenic and non-cardiogenic edema (2). Moreover, to date, no proven drug therapy is available for permeability edema associated with ALI and $\operatorname{ARDS}(2,31,38)$. Morbidity and mortality inversely correlate with AFC capacity in this setting $(42,43)$. The severity of shock in sepsis-induced ARDS is associated with lower AFC (44).

As mentioned above, $56 \%$ of patients with permeability pulmonary had an impaired AFC, and only $13 \%$ a maximal AFC rate (Figure 1). Survival of patients with maximal alveolar clearance rate was higher, as compared to patients with abnormal clearance rate, and the days on mechanical ventilation was less in this group. Clinically impressive is also a series of post-lung transplant patients showing a relation between total ischemic time and the degree of post-transplantation protein-rich and

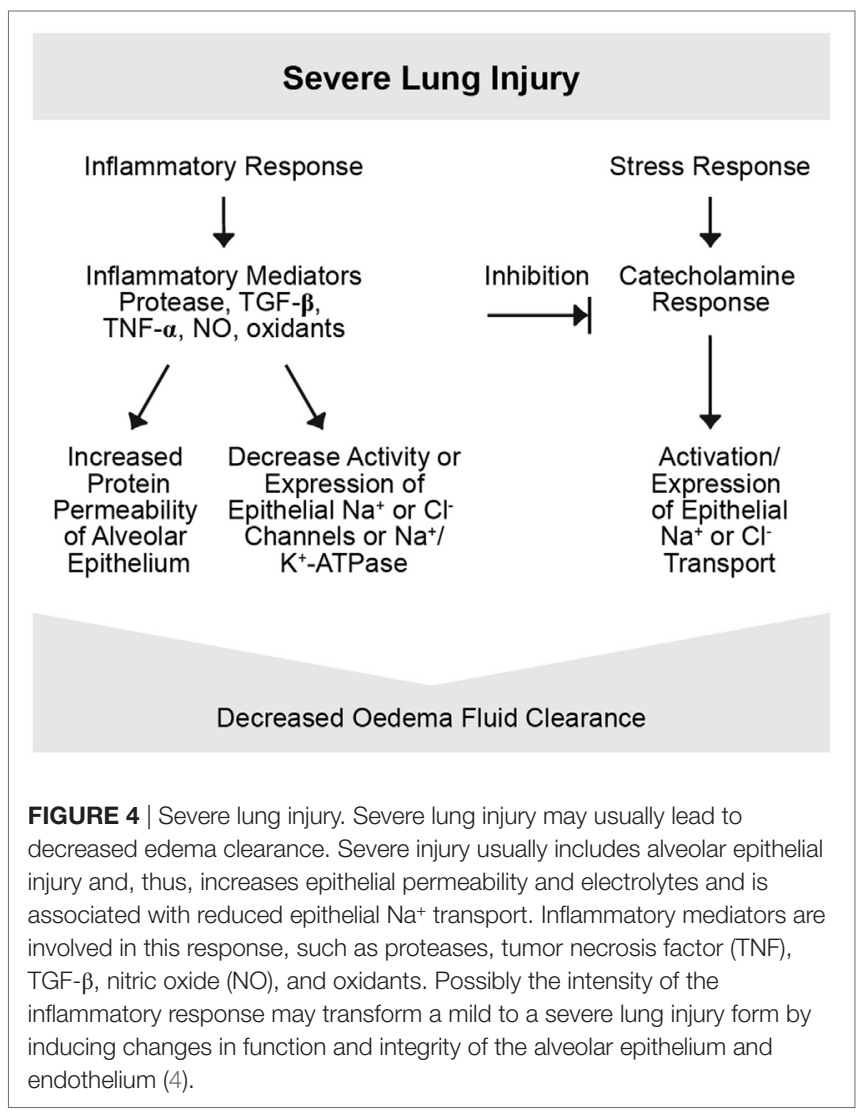


highly neutrophil-rich (71-99\% of cells) permeability edema. Those patients with the best AFC had the best clinical outcomes, including the least and the fastest resolving pulmonary reimplantation response (45). Thus, the ability to reabsorb fluid from the alveolar space was a marker of less severe reperfusion injury. These findings indicate that intact alveolar epithelial fluid transport is critically important for a timely recovery from posttransplantation reperfusion pulmonary edema.

\section{PULMONARY FLUID BALANCE THROUGH BARRIERS}

Airways normally have a critically regulated fluid layer essential for normal gas exchange and removal of foreign particulates from the airway. Maintaining this fluid layer in the alveoli also depends critically on sodium reabsorption. The pulmonary epithelium serves as a barrier to prevent access of the inspired luminal contents to the subepithelium (11) and modulates the initial responses of the airways and lung to both infectious and non-infectious stimuli (11). One mechanism by which the epithelium achieves this is by coordinating transport of diffusible molecules across the epithelial barrier, both through and between cells (11). Specific elements of pulmonary alveoli play different roles as a barrier maintaining the pulmonary fluid balance (38). These barriers will be discussed in more detail below.

\section{Epithelial Barrier}

Lung epithelium is a mucosal surface composed of ciliated cells, mucus-producing cells, and undifferentiated basal and progenitor cells. This dynamic barrier forms the interface between the lumen and the parenchyma from the upper airways to the alveoli. The lung epithelium constantly responds to luminal stimuli and coordinates its response to maintain homeostasis in the lung (11). A breakdown in this coordinated response can cause different lung diseases (11). The alveolar epithelium $(0.1-0.2 \mu \mathrm{m})$ covers $99 \%$ of the airspace surface area in the lung (46) and contains a number of important cell types. Type I cells (AT1) cover at least $95 \%$ of the alveolar surface and are the apposition between the alveolar epithelium and the vascular endothelium. This provides a tight barrier that facilitates efficient gas exchange and which is involved in fluid and protein movement from the interstitial and vascular sites $(38,47)$ and its reabsorption vice versa $(4,5)$. The role of aquaporin $5(\mathrm{AQ}-5)$ in AFC is not clear, in view of the normal AFC capacity in physiological situations in AQ-5 knock out mice (48). The osmotic clearance of water secondary to the ion transport gradient across the alveolar epithelium probably occurs by paracellular pathways and not by the assumed transcellular using aquaporin 5 (25); however, their role in injury is not fully excluded (4). Type II cells (AT2) cover about 5\% of the alveolar surface and are known especially for their key function in surfactant secretion and in vectorial transport of $\mathrm{Na}^{+}(49)$, a major driving force for fluid removal from the alveolar space. Amiloride-sensitive sodium channels on the apical, "air-faced," surface, mainly the $\mathrm{ENaC}$, are key channels in alveolar fluid transport $(50,51)$, with the driving force stemming from the $\mathrm{Na}^{+} / \mathrm{K}^{-}$-ATPase on the basolateral, "blood-faced," surface (46). Dysfunction of these $\mathrm{Na}^{+}$transporters during inflammation can contribute to pulmonary edema (52-54). Tight junctions (TJ) that connect adjacent epithelial cells near their apical surfaces and maintain apical and basolateral cell polarity are fundamental to create a permeability barrier required to preserve distinct compartments in the lung (55).

Alveolar and distal airway epithelia are surprisingly resistant to injury, particularly if compared to the adjacent lung endothelium. When lung endothelium gets injured, the alveolar epithelial barrier may retain its normal impermeability and its normal fluid transport capacity, as seen in animal models with LPS given intravenously or intratracheally (4). This might explain why in mild-to-moderate lung injury AFC may not only be preserved, but even upregulated by stress hormones-an effect that may be inhibited by amiloride or propranolol.

However, in severe ALI, ARDS, and pneumonia, epithelial cell death may occur, as has been shown in a seminal morphological study published 4 decades ago by Bachofen and Weibel (56). A central role for soluble Fas ligand (FasL) has been proposed in AT1 and AT2 cell death, and an association between its levels in bronchoalveolar lavage level on day 1 of ARDS and patient death has been proposed $(57,58)$. However, there may be extensive crosstalk between injurious, inflammatory, and death cascades and repair in the lungs, as well as in other organs in patients with ARDS. Direct alveolar cell death may probably also occur due to bacterial exotoxins or stresses like overdistension. Such epithelial cell death may make the lungs prone to increased permeability and thus disturb AFC, as well as to the danger of disordered repair, such as in fibroproliferative ARDS.

Recent work on different predictors of ARDS suggests that the degree of AT1 cell injury is a central determinant of outcome in ALI and ARDS. Receptor for advanced glycation end products (RAGE) is an immunoglobulin superfamily member, involved in propagating inflammation. RAGE is abundant in the lungs and can be primarily found in AT1 cells. Higher baseline plasma levels of RAGE were found to be associated with worse outcome, including less ventilator-free days and increased mortality, and it excellently discriminated in sepsis patients for the diagnosis of ARDS. Higher levels in bronchoalveolar lavage also predicted post-lung-transplant primary graft failure and correlated with its grade of severity (59). Apart from RAGE, also surfactant protein D level, an AT2 cell product, was, together with the neutrophil chemokine IL-8 (CXCL8), the best performing biomarker for poorer outcome in terms of mortality (60).

\section{Endothelial Barrier}

The capillary endothelial barrier also functions as a key component to maintain the integrity of the vascular boundaries in the lung. The gas exchange surface area of the alveolar-capillary membrane is extremely huge and optimized to facilitate perfusion-ventilation matching (61). Pulmonary endothelium separates also the intravascular marginated pool of polymorphonuclear neutrophils from the airspaces. The endothelium, the most abundant cell relative to the total cell population in the lung, has additional key regulatory roles apart from gas exchange, 


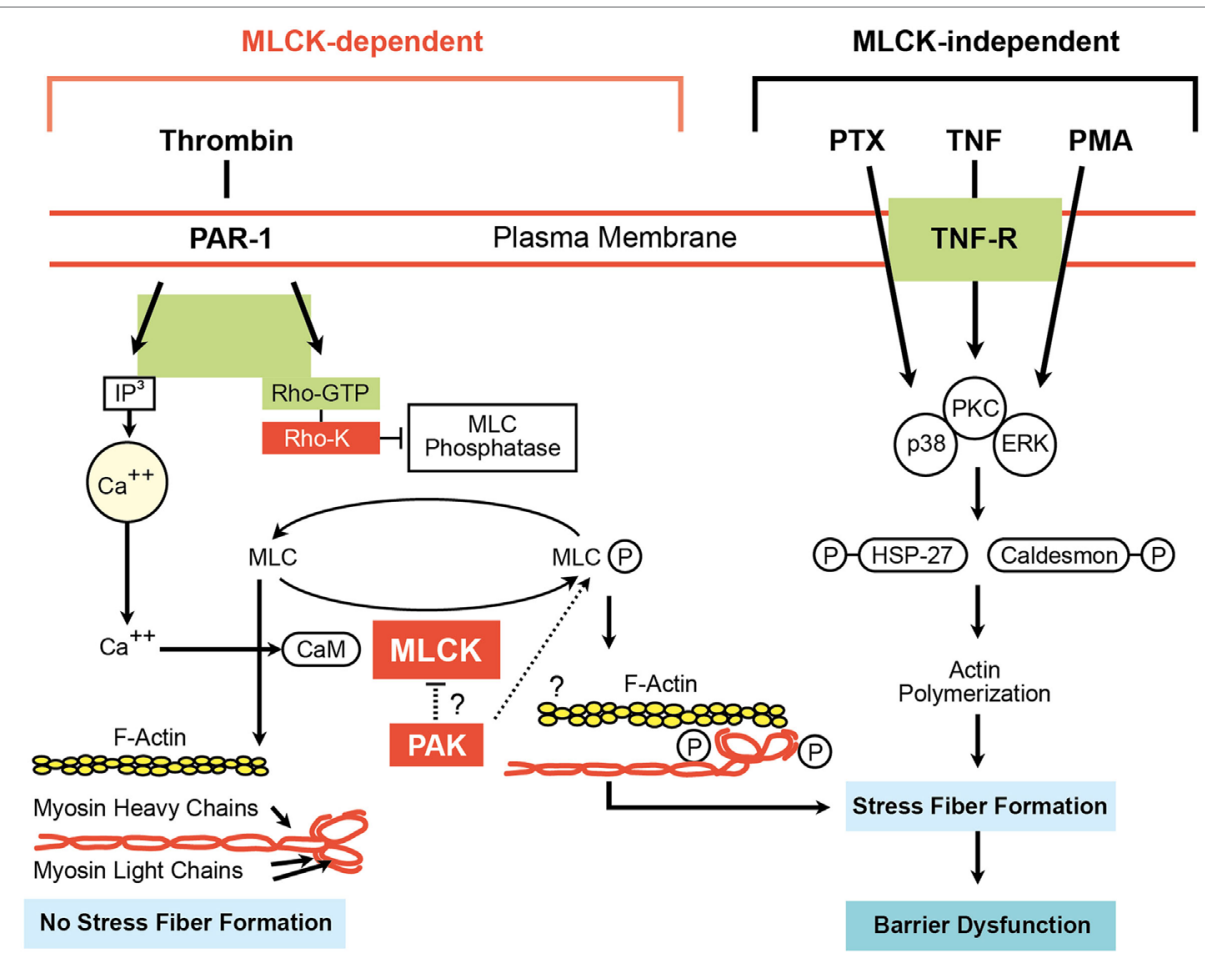

FIGURE 5 | Myosin light chain kinase (MLCK)-dependent and MLCK-independent pathways involved in endothelial cell (EC) barrier dysfunction (66). Adapted from Ware and Matthay (2).

namely vascular tone via nitric oxide (NO) and endothelin-1, and coagulation, as recently discussed in depth in a review on the endothelium and ARDS (34).

In the pulmonary microvasculature, the endothelial cells (ECs) form a semi-permeable barrier between the blood and the lung interstitium (38). Disruption of this barrier may occur during inflammatory disease such as pneumonia, ALI, ARDS, or ischemia-reperfusion injury. In sepsis, early microcirculatory perfusion indices are more markedly impaired in non-survivors, as compared to survivors and correlate with increasing severity of vascular dysfunction (62). Lung ECs are considered orchestrators of the inflammatory response. These cells can directly sense pathogens via toll-like receptors and may contain local bacterial spreading by coagulation, leading to capillary thrombosis and extravascular fibrin deposition (34). This contributes to an increased dead-space fraction that correlates with clinical outcome (63). In sepsis, overwhelming EC activation can lead to apoptosis within minutes to hours (64), which in turn increases barrier permeability and subsequent mortality (65). In ARDS, EC death can occur in by mechanical insults, like shear stress, and by pro-inflammatory mediators, including TNF, angiostatin, and TGF- $\beta$ (39).

Intercellular junctions act as dynamic structures and do not statically resist entry to all substances. They that can open or close in response to physiological or pathological stimuli. Figure $\mathbf{5}$ presents some potential pathways regulating EC barrier function (66). Endothelial barrier dysfunction can result in the movement of both fluid and macromolecules into the interstitium and pulmonary air spaces. This can contribute to important morbidity and mortality (66). TNF can reduce capillary endothelial barrier function $(67,68)$.

\section{REGULATION OF AFC}

In the normal lung, fluid and protein leakage is thought to occur primarily through small gaps between capillary ECs $(2,3)$. Since both capillary endothelial and AECs have TJ, fluid, and macromolecules that are filtered from the circulation into the alveolar interstitial space normally do not enter the alveoli (2).

The hydrophobic plasma membranes composed of phospholipids, act as a huge energy barrier for transporting ions (69-71). Yet, physiological processes assure for the continuous in- and outflow of ions, as such overcoming the plasma membrane barrier, which is impermeable to ions. Due to their biological complexity, interactions between cytokines and ion channels may be under-recognized (72). A group of plasma membrane proteins, including active transporters, generate and maintain ion concentration gradients for particular ions. These active transporters carry out this task by forming complexes with the ions they are translocating. The process of ion binding and unbinding for transport typically requires several milliseconds. As a result, ion translocation by active transporters is much slower than ion movement through ion channels, which can conduct thousands of ions across a membrane each millisecond. Active transporters effectively store energy in the form of ion concentration gradients, whereas the opening of ion channels 
rapidly dissipates this stored energy during relatively brief electrical signaling events.

Several types of active transporters have now been identified. Although the specific roles of these transporters differ, all must translocate ions against their electrochemical gradients (energetically "uphill”). Moving ions uphill requires the use of energy, and neuronal transporters fall into two classes based on their energy sources. Some transporters acquire energy directly from the hydrolysis of ATP and are called ATPase pumps. The most prominent example of an ATPase pump is the $\mathrm{Na}^{+} / \mathrm{K}^{+}$-ATPase pump, which is responsible for maintaining transmembrane (TM) concentration gradients for both $\mathrm{Na}^{+}$and $\mathrm{K}^{+}$(73). Another one is the $\mathrm{Ca}^{2+}$ pump, which provides one of the main mechanisms for removing $\mathrm{Ca}^{2+}$ from cells. The second class of active transporters does not use ATP directly as an energy source, but rather the electrochemical gradients of other ions. This type of transporter carries one or more ions up its electrochemical gradient, while simultaneously taking another ion, most often $\mathrm{Na}^{+}$, down its gradient. These transporters are usually called ion exchangers. An example of such a transporter is the $\mathrm{Na}^{+}$/ $\mathrm{Ca}^{2+}$ exchanger, which shares with the $\mathrm{Ca}^{2+}$ pump the important task of keeping intracellular $\mathrm{Ca}^{2+}$ concentrations low. Other exchangers regulate both intracellular $\mathrm{Cl}^{-}$concentration and $\mathrm{pH}$ by swapping intracellular $\mathrm{Cl}^{-}$for another extracellular anion, bicarbonate, or the $\mathrm{Na}^{+} / \mathrm{H}^{+}$exchanger that regulates intracellular $\mathrm{pH}$, by regulating the concentration of $\mathrm{H}^{+}$. Although the electrochemical gradient of $\mathrm{Na}^{+}$(or other counter ions) is the immediate source of energy for ion exchangers, these gradients ultimately depend on the hydrolysis of ATP by ATPase pumps, such as the $\mathrm{Na}^{+} / \mathrm{K}^{+}$ATPase pump (74).

Alveolar fluid clearance is mainly regulated by $\mathrm{Na}^{+}$uptake through the apically expressed $\mathrm{ENaC}$ and the basolaterally localized $\mathrm{Na}^{+} / \mathrm{K}^{+}$-ATPase in type II AECs (Figure 2, lower panel) (54). Dysfunction of these $\mathrm{Na}^{+}$transporters during pulmonary inflammation can contribute to pulmonary edema (54). In this context, the movement of larger plasma proteins is restricted (2). The hydrostatic force for fluid filtration across the lung microcirculation is approximately equal to the hydrostatic pressure in the pulmonary capillaries, which is partly compensated by a protein osmotic pressure gradient (2). The net quantity of accumulated pulmonary edema is logically determined by the balance between the rate at which fluid is filtered into the lung (1) and the rate at which fluid is removed from the air spaces and lung interstitium (46). In mild-to-moderate lung injury, the capacity of the alveolar epithelium to transport salt and water is not only preserved but may also even be upregulated by stress hormones (Figure 3) (4). In severe lung injury, pulmonary fluid clearance can also be stimulated in lung injury by catecholamine-independent mechanisms (Figure 4) (4).

Moderate hypoxemia was shown to reduce AFC by $50 \%$. This is caused by decreasing apical sodium uptake, at least partially through impaired trafficking of $\mathrm{ENaC}$ to the surface membrane (75-77). Hypoxia, moreover, inhibits the function of $\mathrm{Na}^{+} / \mathrm{K}^{+}$-ATPase in AECs, in part by triggering endocytosis through reactive oxygen species (ROS) and phosphorylation of the $\alpha 1$ subunit (78) (Figure 6). Restoration of normoxia rapidly reversed the depressant effects of hypoxemia in rats. Therefore, the simple administration of supplemental oxygen to patients with pulmonary edema may enhance the resolution of alveolar edema. As discussed more in detail in a contribution by Vadasz and Sznajder in this topic issue, hypercapnia can also impair AFC by the mechanisms of ubiquitination-mediated retrieval of $\mathrm{ENaC}$ from the plasma membrane, i.e., a post-translational modification of $\beta \mathrm{ENaC}$ by regulating trafficking and stability, thereby modifying, and in this case reducing cell surface expression of the channel through $\beta \mathrm{ENaC}$ ubiquitinylation in the alveolar epithelium (78-80). This mechanism seems of importance in ARDS as well in COPD. Hypercapnia and the associated acidosis have been shown to have anti-inflammatory effects, which might be advantages at sites of excessive inflammation, whereas

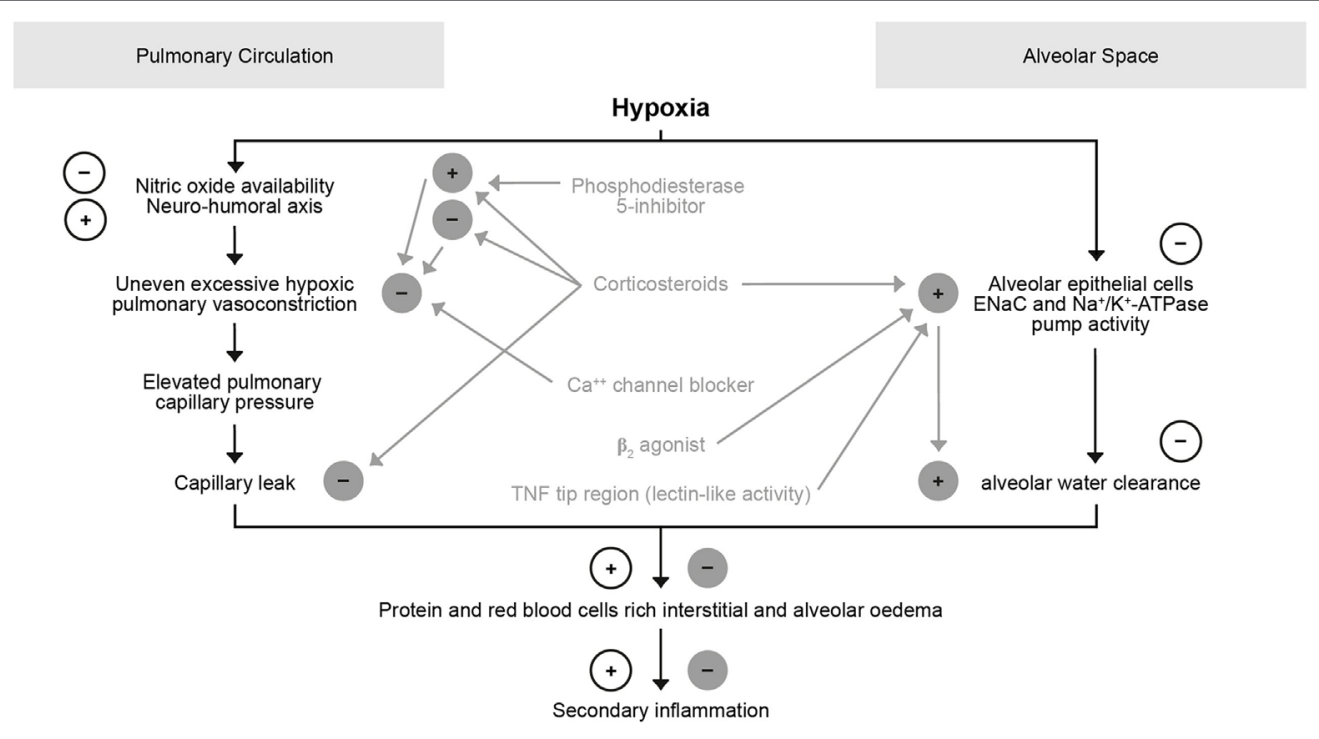

FIGURE 6 | Role of hypoxia in the pulmonary circulation and alveolar space. 
on the other hand, ARDS and COPD studies showed that both patient groups had worse outcome when they were hypercapnic (78). In a randomized controlled trial Köhnlein, Windisch et al. showed that in severely sick, chronic hypercapnic COPD patients non-invasive ventilation, when targeted to reach noromocapnia $(\mathrm{PaCO} 2<6.5 \mathrm{kPa} / 48.1 \mathrm{mmHg}$ ) or to improve hypercapnia by at least $20 \%$, is associated with much better outcome (81). Survival was impressively improved, and also quality of life and lung function in terms of FEV1 improved. Possibly further effects exist such as sometimes improved cardiac output $(82,83)$, although interactions between ventilation and cardiac output are complex.

\section{Ion Channels and Pumps/Transporters and AFC}

Ion channels are integral membrane proteins that form a pore to allow the passage of specific ions by passive diffusion (84). Most ion channels undergo conformational changes from closed to open states. Once open, ion channels allow the passage of thousands of ions (84). This distinguishes them from transporters and pumps, which can also transport ions, but only a few at a time (84). The opening and closing of channels can be controlled by various means, including voltage, the binding of ligands such as intracellular $\mathrm{Ca}^{2+}$ or extracellular neurotransmitters, and post-translational modifications such as phosphorylation (84).

Ion channels and pumps also play multiple important roles in cell homeostasis (84). Their function promotes passive, agonistinduced, or voltage-dependent flux of specific ions in and out of the cell $(84,85)$. The mchanisms of removing the infiltrated fluid from the alveoli is called AFC (84).

\section{The ENaC in Type I and II Alveolar Epithelial Cells}

Epithelial sodium channel, a member of the $\mathrm{ENaC} /$ degenerin (ENaC/DEG) family of ion channels, constitutes the ratelimiting entry step in $\mathrm{Na}^{+}$reabsorption across epithelial in colon, kidney, and lungs (86). ENaC is inhibited by the drugs amiloride, benzamil, and triamterene, some of which are clinically used as potassium-sparing diuretics $(87,88)$. ENaC is a heteromultimeric protein (89) and is composed of at least four homologous subunits, $\alpha, \beta, \gamma$, and $\delta$ (89-91) which are able to compose an ion channel $(50,92)$. A functional, pore-forming channel usually comprises one or two $\alpha$ subunits, together with a $\beta$ - and a $\gamma$ -subunit $(89,91,93,94) . \delta$ as a fourth unique subunit can form ion channels joining the $\beta$ and $\gamma$ subunits but exhibits biophysical and pharmacological features that are different compared to $\alpha \mathrm{ENaC}$ channels (95). Investigations of the biological role of $\alpha \mathrm{ENaC}$ in the mouse lungs underlined the crucial role of this subunit in AFC (12). The $\beta$ subunit is highly glycosylated and an important regulator of $\mathrm{ENaC}$ (4). In the lungs, $\mathrm{ENaC}$ is expressed not only in alveolar type II and type I cells (96), but also in capillary ECs (97).

Epithelial sodium channel was shown to exert a crucial role in pulmonary fluid reabsorption (46). Accordingly, $\mathrm{ENaC}$ is responsible for the maintenance of $\mathrm{Na}^{+}$balance, extracellular fluid volume and blood pressure (98). ENaC activity is determined by the number of channels in the surface membrane $N$, which can change according to membrane insertion, degradation, or retrieval, as well as by the open probability time Po of individual channels $(86,99,100)$. The basolaterally expressed, ouabain-inhibitable $\mathrm{Na}^{+} / \mathrm{K}^{+}$-ATPase then further drives the vectorial transport into the interstitium and, finally, into the lymphatic and blood vessels (73).

In order to maintain the correct composition and volume of alveolar lining fluid, $\mathrm{Na}^{+}$transport through apically located $\mathrm{ENaC}$ in the alveolar epithelium is critical for gas exchange (92).

Epithelial sodium channel expression was shown to be decreased in transplanted lungs, both at the messenger RNA and protein level $(8,101)$.

\section{Physiological ENaC Regulation}

Epithelial sodium channel activity is important for fluid homeostasis and blood pressure control, but its regulation is complex and remains in many aspects incompletely understood (102) (Table 1). ENaC channels are also called highly selective cation (HSC) channels, and are presumed to be made up by the three $\mathrm{ENaC}$ subunits, $\alpha, \beta$, and $\gamma$ (103).

Epithelial sodium channel function can be affected by direct modulation of channel activity (92), subunit degradation, and membrane trafficking/recycling (104). cAMP indirectly increases ENaC activity, since it activates $\mathrm{Cl}^{-}$uptake through CFTR (105). Intracellular as well as extracellular proteases, including prostasin and furin can affect the activity of the channel by modulating the $\mathrm{Na}^{+}$self-inhibition $(106,107)$. Another important system that modulates $\mathrm{ENaC}$ activity is trafficking of the channels to the membrane, which involves a complex system of ubiquitination and binding to Nedd-4-2 (108). $\mathrm{Na}^{+}$transport can also be regulated by gene expression (4). The two major hormonal modulators of pulmonary $\mathrm{ENaC}$ expression are catecholamines (50) and corticosteroids (109).

Many agents that increase $\mathrm{Na}^{+} / \mathrm{K}^{+}$-ATPase activity also increase $\mathrm{ENaC}$ activity (36). Negative $\mathrm{ENaC}$ regulators are activated purinergic $\mathrm{P} 2 \mathrm{Y}$ receptors (110), NO $(111,112)$, Il-1 $\beta$ (113), hypoxia (46), and TGF- $\beta$ (46).

\section{ENaC Dysfunction}

Dysfunction of the ENaC, which regulates salt and water homeostasis in epithelial, causes several human pathological conditions, including pulmonary edema (114). As ENaC regulates the airway surface liquid layer, its exaggerated activity might lead to airway dehydration, mucus stasis and bacterial overgrowth, as can be seen in cystic fibrosis and chronic bronchitis (115-117). ENaC hypo-activity, by contrast, can dramatically impair AFC, which is particularly important in conditions of pulmonary edema and correlates with mortality and morbidity in patients with ALI and ARDS (33).

The significant role of $\mathrm{ENaC}$ in inherited diseases associated with mutations in $\mathrm{ENaC}$ which increase or decrease channel activity regarding salt and water homeostasis has been welldocumented (118). Mutations in the PPxY motif of $\beta$ - and $\gamma$-subunits cause a severe form of hypertension, associated with ENaC in Liddle's syndrome (OMIM: 177200) (119-123). A decrease in $\mathrm{ENaC}$ function can also cause a rare, life-threatening salt-wasting syndrome in pseudohypoaldosteronism type $1 \mathrm{~B}$ 
(PHA1B) (OMIM: 264350) (124-127). This disease does not improve with age and patients are at risk from life-threatening, salt-losing crises, combined with severe hyperkalemia and dehydration throughout their entire lives $(128,129)$. Additionally, dysregulation of channel function and/or expression can lead to organ dysfunction and severe disease $(84,85,130)$.

\section{The Hybrid Acid-Sensing lon Channel 1a (ASIC1a)/ $\alpha$-ENaC (NSC) Channels in Alveolar Type I and Type II Cells}

Apart from $\mathrm{ENaC}$, another apically expressed channel was recently shown to promote AFC. This hybrid channel is relatively non-selective for $\mathrm{Na}^{+}$over $\mathrm{K}^{+}$, has a larger conductance, and shorter mean open and closed times $(103,131)$. In elegant assays, Trac et al. showed that the channel included ASICla as the mandatory counterpart to $\alpha$-ENaC. These hybrid channels are, thus, composed of, at a minimum, one $\alpha-\mathrm{ENaC}$ subunit and one or more ASICla subunits. The biological significance is great, as the regulation of these NSC channels is dramatically different from $\mathrm{ENaC}$. Thus, treatments to reduce alveolar flooding based on the known properties of $\mathrm{ENaC}$ (HSC) could be suboptimal because


Indeed, NSC channels are less sensitive to inhibition by amiloride than ENaC HSC channels.

As the proton-gated ASIC1a plays a role in the formation of channels, its properties determine the pharmacological ASIC1a/ $\alpha$-ENaC-channels (NSC) modulation. The MitTx agonist, derived from Texas coral snake toxin, strongly activates ASICla/ $\alpha$-ENaC-channels (NSC) (Table 3).

\section{Why Do Alveolar Epithelial Cells in the Lungs Have Several Types of Channels That Mediate $\mathrm{Na}^{+}$ Uptake?}

As shown, an important functional role of non-selective cation (NSC) channels, which consist of ASIC1a and of ENaC- $\alpha$ subunits (281), is $\mathrm{Na}^{+}$uptake by AT2 cells in the lung (103). By contrast, other sodium-transporting epithelial tissues such as the distal nephron of the kidney and the colon were not reported to have these functional NSC channels, and mainly transport $\mathrm{Na}^{+}$through ENaC. In the lungs, the alveolar fluid layer must be very tightly controlled. Therefore, it may be important to have alternative ion transport pathways that respond differently to physiological stimuli, such as to acidification, which accompanies ALI and which activates NSC channels (282). An alternative hypothesis is that NSC channels provide a stable driving force for cation and anion movement across the alveolar epithelium. Indeed, NSC channels contribute to the apical membrane potential, causing the membrane potential to be close to zero. This will ensure that there is a driving force for the unidirectional movement of anions, through CFTR and for movement of $\mathrm{Na}^{+}$through classical $\mathrm{ENaC}$ and NSC into cells. This is necessary because of the requirement to move salt, i.e., anions plus cations. Other epithelia tend to have counter-ion pathways for cations that obviate the need to maintain a strong potential driving force.

In an evolutionary context, the lung has been the most recent organ to adapt to a terrestrialenvironment. Typical for evolutionary processes is the modification of existing mechanisms to produce a different evolutionary outcome, in this case, the formation of a new channel type out of parts from two pre-existing channels of the same channel family. Of further evolutionary interest is that the activity of both HSC channels HSC (ENaC) and NSC channels is increased by a peptide mimicking the lectin-like region of TNF, which binds to ENaC- $\alpha$, as shown below and in Czikora et al. (9), in this issue (9).

\section{The $\mathrm{Na}^{+} / \mathrm{K}^{+}$-ATPase}

Apart from apical ENaC and, potentially NSC, the basolaterally expressed $\mathrm{Na}^{+} / \mathrm{K}^{+}$-ATPase, a.k.a. the sodium-potassium pump is also a crucial driver of $\operatorname{AFC}(73,78) . \mathrm{Na}^{+} / \mathrm{K}^{+}$-ATPase activity regulation also involves complex patterns, including modulation of the trafficking of the protein to the membrane (73). The $\mathrm{Na}^{+} / \mathrm{K}^{+}$-ATPase is a ubiquitous enzyme consisting of $\alpha$ and $\beta$ subunits and a less well-characterized regulatory FXYD subunit. The $\mathrm{Na}^{+} / \mathrm{K}^{+}$-ATPase is responsible for the generation and preservation of the $\mathrm{Na}^{+}$and $\mathrm{K}^{+}$gradients across the cell membrane by transporting $3 \mathrm{Na}^{+}$out and $2 \mathrm{~K}^{+}$into the cell (283).

Changes in intracellular $\mathrm{Na}^{+}$concentration and hormones, such as mineralocorticoids, glucocorticoids and thyroid hormones as well as adrenoceptor stimulants modulate $\mathrm{Na}^{+} / \mathrm{K}^{+}$-ATPase activity (284). Like ENaC, increase of $\mathrm{Na}^{+} / \mathrm{K}^{+}$-ATPase expression is considered central to enhance transepithelial $\mathrm{Na}^{+}$transport (4). In addition, thyroid, mineralocorticoid and glucocorticoid hormones modulate $\mathrm{Na}^{+} / \mathrm{K}^{+}$-ATPase expression (4). Likewise, $\beta$ adrenoceptor activation upregulates $\mathrm{Na}^{+} / \mathrm{K}^{+}$-ATPase expression in AECs (50).

The $\mathrm{Na}^{+} / \mathrm{K}^{+}$-ATPase contains one principal catalytic subunit, designated $\alpha$ and one sugar-rich auxiliary subunit, designated $\beta$. There is also a regulatory subunit FXYD subunit, which was recently shown to play an important role in regulation of lung inflammation (285). The $\alpha$-subunit carries the catalytic function of the enzyme, and this is reflected in its possession of several binding and functional domains (283). The $\alpha$ subunit (4) transports $\mathrm{Na}^{+}$out of the cell, providing the driving force for $\mathrm{Na}^{+}$ reabsorption (286). It is clear that an essential role for $\beta$ subunit lies in the delivery and the appropriate insertion of the $\alpha$ subunit in the membrane (287). In recent years, a variety of studies have suggested that the $\beta$ subunit may be more intimately involved in the mechanism of active transport (287-290).

FXYD5 or Dysadherin or RIC is a pro-inflammatory type I membrane protein, which belongs to seven members of the FXYD family named by their shared TM amino acid motif. FXYD5 is an established tissue-specific modulatory subunit of $\mathrm{Na}^{+} / \mathrm{K}^{+}$-ATPase, expressed in a variety of epithelial cells. Recent work shows a role for FXYD5 as a key mediator of the inflammatory response during ALI (285). It impairs adherens junctions by downregulating the markers zona occludins- 1 (ZO-1) and occludin and redistributing beta catenin (291). It is required for the secretion of NF- $\mathrm{KB}$, e.g., upon lipopolysaccharide (LPS), and inflammatory mediators, including TNF and interferon- $\alpha$ (IFN$\alpha$ ) and C-C chemokine ligand-2 (CCL2) from AECs that activate alveolar macrophages, amplify lung injury by orchestrating an overly exuberant inflammatory response, and recruit monocytes into the alveolar compartment, or in bronchoalveolar lavage 
fluid (285). The presence of FXYD5 is an important component for NF- $\kappa \mathrm{B}$ activation pathway as shown in AECs induced by LPS, TNF, or interferon- $\alpha$, as its silencing prevented I $\mathrm{BB}-\alpha$ phosphorylation and reduced cytokine secretion in response to these stimuli. Probably FXYD5 increases CCL2 transcription by inducing Akt-dependent activation of NF- $\kappa B$ signaling. Binding of IFN- $\alpha$ activated phosphoinositide 3-kinase (PI3K) via STAT5, which in turn activates NF- $\kappa$ B. Activation of PI3K seems downstream of TLR4 and TNFR1. Possibly, FXYD5 modulates NF- $\kappa \mathrm{B}$ signaling by regulating the location of TNF receptor 1 , by modulation associations with other proteins and their location and mobility in the membrane (285). It is of interest that FXYD5 regulates inflammation, activates NF- $\mathrm{KB}$ dependent cytokine secretion and infiltration of immune cells to the alveolar spaces as well as alveolar barrier tightness, and is closely linked to one key ion transport channel.

\section{The Cystic Fibrosis Transmembrane Conductance Regulator}

Cystic fibrosis transmembrane conductance regulator is a cAMPregulated and post-translationally modified chloride channel of 1,480 amino acids, which is mainly expressed in epithelial cells. The non-glycosylated form of CFTR has a molecular weight of $127 \mathrm{kDa}$, with $160 \mathrm{kDa}$ for the glycosylated form. CFTR can either take up or release $\mathrm{Cl}^{-}$ions from the AT1 and AT2 cells. Apical to basolateral chloride transport may be important because the maximal rate of sodium and water transport from the airspaces appears to be limited by the concomitant chloride transport (115-117). An important part of transepithelial chloride transport occurs through the paracellular route in the alveolar epithelium. The selectivity and magnitude of paracellular ion conductance may influence net transport capacity. Upon increasing $\mathrm{Cl}^{-}$influx, CFTR will activate ENaC-mediated $\mathrm{Na}^{+}$uptake, as such activating $\mathrm{AFC}$, but the channel will inhibit AFC upon increasing $\mathrm{Cl}^{-}$efflux. Increased cAMP generation will open CFTR in the apical membrane of AT1 and AT2 cells for $\mathrm{Cl}^{-}$uptake, as such increasing $\mathrm{Na}^{+}$uptake and AFC. Therefore, factors that can activate cAMPmediated $\mathrm{Cl}^{-}$uptake by CFTR, such as $\beta 2$ agonists, have been investigated as potential therapeutic candidates for pulmonary edema (105). Cystic fibrosis, a disease characterized by impaired airway dehydration, is caused by a loss of function of CFTR, accompanied by an excessive activity of ENaC. A peptide mimetic of SPLUNC, i.e., SPX-101, was shown to promote internalization of the three $\mathrm{ENaC}$ subunits and to restore mucus transport in a mouse and a sheep model of CF (292).

\section{The Transient Receptor Potential Vanilloid 4 (TRPV4) Channel}

Transient receptor potential vanilloid 4 is a TM cation channel and a vanilloid-type member of the transient receptor potential (TRP) protein superfamily (293). TRPV4 is ubiquitously expressed in many cell types in the respiratory system (294). It is part of an integrated system, consisting of ion channels and membrane pumps, which tightly regulates intracellular calcium levels in a spatiotemporal manner (295). TRPV4 counts 871 amino acids and contains six TM domains, an ion pore located between TM5 and 6, an NH2 terminal intracellular sequence with several ankyrin-type repeats, and a $\mathrm{COOH}$-terminal intracellular tail $(296,297)$. Both the $\mathrm{NH} 2$ and $\mathrm{COOH}$ termini interact with signal kinases, other molecules (e.g., NO), and scaffolding proteins (298). The intracellular tails contain several activity-modifying phosphorylation sites (294). In the setting of pulmonary inflammation, TRPV4 has been found to be highly expressed and upregulated in airway smooth muscle, vascular ECs, AECs, as well as in immune cells, such as macrophages and neutrophils (298-303).

\section{The Role of TRPV4 in Pulmonary Edema}

Transient receptor potential vanilloid 4 mediates cellular responses to both physical (such as osmotic, mechanical, and heat) as well as chemical stimuli (304). It is also involved in lung diseases associated with parenchymal stretch and inflammation or infection $(254,294)$. Target diseases include cough, asthma, cancer, and pulmonary edema associated with ARDS (253, 294, 305-310).

These studies support a role for TRPV4 in a broad spectrum of lung and airway functions and disease processes. TRPV4 also has been implicated as a key regulator of lung endothelial barrier integrity, specifically, the integrity of the lung alveolar-capillary endothelium, which is most relevant to alveolar edema generation in ALI (311). TRPV4 activation increases vascular permeability, thus promoting protein and fluid leak (254).

Several studies have shown that TRPV4 can regulate generation of inflammatory cytokines that play key roles in orchestrating lung tissue homeostasis and inflammatory lung disease (301, 307, 309, 310, 312-314). Therefore, TRPV4 could be considered a potential target for lung disease pathogenesis, including to alveolar-capillary barrier function (300). TRPV4 has been proposed as a candidate target for the management of ALI that develops as a consequence of aspiration of gastric contents, or acute chlorine gas exposure (254). Protection from the ALI response to intratracheal $\mathrm{HCl}$ and a key role in vivo of polymorphonuclear neutrophil TRPV4 (294) was noted in mice that lack TRPV4 (TRPV4 KO), or in mice that were treated with three different small molecule inhibitors of TRPV4 (253, 301, 307, 309, 312, 313, 315).

However, in view of its ubiquitous expression, and the multitude of functions attributed to the channel, including its role in pulmonary vasomotor control, endothelial barrier tightness, inflammatory response and systemic blood pressure regulation, TRPV4 blockade may represent a double-edged sword. Therapeutic benefits of TRPV4 inhibition have, therefore, to be carefully weighed against potential adverse effects (254).

Transient receptor potential vanilloid 4 activation and its downstream signaling pathways differ in response to varying stimuli, cell types, and contexts (294). For instance in asthma, TRPV4 mediates hypotonicity-induced airway hyperresponsiveness, but not release of Th2 cytokines $(312,316)$. In CF, TRPV4 appears to play paradoxical roles in $\mathrm{CBF} /$ mucociliary clearance and epithelial cell pro-inflammatory chemokine (IL-8/KC) secretion $(317,318)$. Depending on the underlying etiology, TRPV4 may play different roles in ARDS (307, 310, 314, 319). Also, in pulmonary fibrosis, TRPV4 has been shown to mediate the 
mechano-sensing that drives myofibroblast differentiation and experimental lung fibrosis in mice (308).

\section{TRPV4 and Macrophage Function in Lung Injury}

Alveolar macrophages are known to be effector cells in bacterial and particle clearance but also in any injury and repair process (320). Since intracellular $\mathrm{Ca}^{2+}$ is known to be required for the phagocytic process, and because TRPV4 plays a role in forcedependent cytoskeletal changes in other systems/cell types, the role of TRPV4 in macrophage phagocytosis was extensively studied by Scheraga and colleagues (213, 253, 307, 315, 321-323). The process of phagocytosis in macrophages requires integration of signals from macrophage surface receptors, pathogens, and the extracellular matrix (324-326). However, the effects of matrix stiffness on the macrophage phenotypic response or its signal transduction pathways have yet to be fully elucidated (294). TRPV4 mediates LPS-stimulated macrophage phagocytosis of both opsonized particles [immunoglobulin $\mathrm{G}$ (IgG)-coated latex beads] and non-opsonized particles (Escherichia coli) in vitro (294). Inhibition of TRPV4 by siRNA or pharmacologic inhibitors completely abrogated both the LPS effect and the matrix stiffness effect on phagocytosis (294). These data indicate that both the LPS and stiffness effect on macrophage phagocytosis are TRPV4 dependent (310). Concordant with their in vitro data, also LPS-induced alveolar macrophage phagocytosis was proposed to be TRPV4 dependent (294).

Collectively, obtained data demonstrate that TRPV4 responds to extracellular matrix stiffness, thereby altering the LPS signal to mediate macrophage phagocytosis and cytokine production (310). Furthermore, TRPV4 regulates a feed-forward mechanism of phagocytosis in activated lung tissue macrophages when they interact with stiffened infection/injury-associated lung matrix. This concept is further supported by the observation that surfactant protein B-deficient mice have altered alveolar macrophage shape and function in association with increased alveolar surface tension (327).

\section{Other Ion Channels}

Recent research has given much more detail to a number of further ion channels and their interactions, such as $\mathrm{Cl}^{-}$regulators in the paracellular TJ area including claudin- 4 and -18 implicated in epithelial ion and fluid transport and ARDS regulation in specific infectious, inflammatory, or other stimulatory situations. The reader is referred to further reviews as that of Brune et al (11). and Weidenfeld and Kübler (5). The transient receptor potential channel 6 (TRPC6), a $\mathrm{Ca}^{2+}$-permeable non-selective cation channel, widely expressed in the lungs, was proposed to be a key regulator of acute hypoxic pulmonary vasoconstriction and was demonstrated to be implicated in pulmonary hypertension. TRPC6 is also involved in pulmonary vascular permeability and lung edema formation during LPS- or ischemia/reperfusioninduced ALI as discussed in this topic issue (328).

\section{CYTOKINE-ION CHANNEL INTERACTION}

Cytokines, which are organized in a cytokine network, play a major role in maintaining lymphocyte and leukocyte homeostasis under both steady-state and inflammatory conditions (329). Regulatory cytokines have to function in combination with other environmental signals to properly modulate the function and the extent of lymphocyte and leukocyte activation (329). Increased generation of pro-inflammatory cytokines represents a first-line defense mechanism against bacterial infections of the lung (102). Dysregulation of cytokine generation leads to alterations in cellcell interactions (330). Cytokines, such as TNF, IL-1, IL-6 activate host defense by promoting the production of a wide spectrum of other cytokines and chemokines, including GM-CSF, G-CSF and IL-8 in inflammatory processes $(331,332)$. They moreover mediate the increase of surface adhesion molecule expression through activation of leukocytes and ECs (38). As such, cytokines can contribute to the pathogenesis and development of pulmonary edema $(37,99,333-338)$. During the acute phases of ARDS, higher levels of TNF were detected in the BALF from patients with early-stage ARDS (39).

\section{The Dichotomous Yin and Yang Effects of TNF in Pulmonary Edema}

Tumor necrosis factor is a homotrimeric $51 \mathrm{kDa}$ protein, binding to two types of membrane receptors: TNF receptor 1 , which signals either apoptosis, necroptosis or inflammation; and TNF receptor 2, which is mainly implicated in inflammation and which is devoid of a death domain $(239,339,340)$. TNF is one of the central cytokines in inflammation and moreover modulates ion channel activity (341-344). An intriguing feature of the ligands of the TNF and TNFR family is that when certain members are shed, they inhibit the function of the ligand-receptor complex and act as inhibitors (345). A central regulatory process may, therefore, be the proteolytic release of soluble bioactive oligomers from membrane-bound forms, e.g., for TNF by the protease TACE. The existence of TM forms of most of the TNF-superfamily ligands indicates that they are meant to act locally. Only under non-physiological conditions, when these ligands are released, they may prove to be harmful (345) or beneficial, as is the case of immune defense to bacterial infection (346). As a consequence, long-term treatment with TNF neutralizing substances can cause increased sensitivity to tuberculosis (346).

Tumor necrosis factor contributes to the pathogenesis and development of pulmonary edema (38), but, paradoxically, also plays an important role in edema reabsorption (347-350). It was assumed for a long time that cytokines exert their activities solely upon activating their respective receptors, but in the case of TNF, this is not true, which broadens this concept (38). TNF was shown to exert a lytic, i.e., killing effect on certain bloodstream stages of African trypanosomes, by means of a lectin-like interaction with trimannoses and $N, N^{\prime}$-diacetylchitobiose oligosaccharide residues in the variant surface glycoprotein on the surface of the parasites (344). Later investigations could demonstrate that this lectin-like activity can be attributed to a special 17 amino acid long domain, named the lectin-like domain of TNF in the molecule's tip region $(351,352)$ (Figure 7$)$. This special region is spatially distinct from its receptor binding sites (353) and is not present in lymphotoxin, which has a highly similar tertiary structure as TNF. Comparative sequence analysis of TNF and 




FIGURE 7 | Tumor necrosis factor. Tumor necrosis factor (TNF) as a "moonlighting" or dual role, or dichotomal yin-yang cytokine. The TNF receptor 1 binding sites within the TNF homotrimer mediate edema formation and blunt edema reabsorption. The lectin-like domain of the same cytokine activates epithelial sodium channe function and as such promotes alveolar fluid clearance and acts on endothelial cell barrier tightness (360).

LT allowed for the identification of the lectin-like domain of TNF (353).

For experimental purposes to mimic the TNF lectin-like domain, the amino acid sequence-identic synthetic 17 amino acid peptide which has shown to biologically mimic the lectin-like tip domain of TNF (353-355), as described above, has been used in a variety of experimental researches. It, moreover, gave rise to a therapeutic candidate that was recently evaluated in clinical trials (a.k.a AP301 and Solnatide) (356-358).

There are conflicting data about the critical involvement of TNF in the regulation of AFC (359). In situ and in vivo investigations conducted by Braun et al. in flooded rat lungs demonstrated a dual role for TNF in pulmonary edema $(37,38)$. This is possibly due to the opposite effects of, on the one hand, the classical TNF receptor 1 binding sites and, on the other hand, the lectin-like domain of TNF on pulmonary fluid reabsorption (37). In fact, the TNF tip region with its lectin-like activity is spatially distinct from the cytokine's receptor binding sites and causes an increase of alveolar fluid reabsorption, which is completely independent of the TNF receptors type 1 and 2, and further increases the cell-cell barrier tightness as shown in the alveolar EC barrier (Figure 7) $(38,99)$.

As discussed more in detail in this issue (361), in murine models of ventilator-induced ALI, TNF receptor 2 can have protective effects, whereas TNF receptor 1 is deleterious, thus adding another level of complexity to the role of TNF in edema (362). As such, the complex between soluble TNF receptor 1 and TNF can stimulate fluid reabsorption. TNF causes receptor-mediated edema formation in part by decreasing the expression of $\mathrm{ENaC}$ mRNA in AECs in vitro (135) leading to decreased amiloridesensitive sodium uptake (135). Moreover, TNF receptor 1 signaling initiates the process of neutrophil migration (363) which can also contribute to the formation of pulmonary edema. It is also involved in orchestrating mechanisms, such as complement activation, cytokine regulation, chemokine production, and activation of adhesion molecules as well as their respective adhesion molecule receptors (364).

A TNF-dependent and amiloride-sensitive increase in AFC occurs in a rat model of Pseudomonas aeruginosa pneumonia (365). Other studies have shown in rats that intestinal ischemiareperfusion leads to stimulation of AFC. This stimulation is at least in part mediated by a TNF-dependent mechanism which is independent of catecholamine release, because propranolol did not influence the AFC, and there was no observed cAMP stimulation (366). This indicates a protective effect of TNF-dependent stimulation of AFC in the early phase of injury (366).

Fukuda et al. could show that in ventilated rats TNF increased AFC by about $67 \%$ (136). This increase was inhibited by amiloride, but not by propranolol, indicating the mechanism is catecholamine-independent. A triple TNF mutant, in which 
three crucial residues for the lectin-like activity were mutated to alanines, did not show any increase in AFC. The effect of TNF occurred within $30 \mathrm{~s}$ from the onset of perfusion in A549 cells and within $1 \mathrm{~h}$ in the distal airspaces of the rat. This shows that the primary mechanism does not depend on a transcriptional effect of TNF. This indicates that TNF increased AFC most probably by an amiloride-sensitive mode of action, independent of any TNF receptor binding and mediated through the lectin-like region.

These antagonistic functions of the same molecule on pulmonary edema refer to the complex biology of the TNF molecule (361). Indeed the TNF receptor 1 binding sites of TNF inhibit, whereas its lectin-like domain activates edema reabsorption (Figure 7) (37), and, as described above, tightens intercellular epithelial and endothelial barrier function $(8,9)$.

\section{The Impact of TNF on Pulmonary Edema Generation by TNF Receptor-Mediated Effects}

Tumor necrosis factor is mainly known for its receptor-mediated pro-inflammatory functions in the systemic inflammatory response and the induction of apoptosis on a cellular level $(339,367)$. Both of these activities of TNF are implicated in the pathogenesis of pulmonary edema, which is often associated with ALI (37).

Tumor necrosis factor promotes pulmonary dysfunction through edema formation and inhibition of edema reabsorption by several procedures (37), for instance:

- TNFR-dependent upregulation of chemokine production $(338,363)$ and adhesion molecule expression $(333,334,368)$, which leads to neutrophil attraction and sequestration.

- Decrease in barrier function in human pulmonary artery ECs and rearrangement of microtubules (67).

- Induction of reactive oxygen intermediates (336).

- Down-regulation of $\mathrm{ENaC}$ expression in alveolar type 2 cells (135)

TNF Inhibits Transcription of All Three ENaC Subunits Seminal studies conducted by Dagenais et al. clearly demonstrated the involvement of TNF in modulation of $\mathrm{Na}^{+}$absorption in cultured AECs is investigated. The results show that TNF decreased the expression of the $\alpha$-, $\beta$-, and $\gamma$-subunits of ENaC mRNA after 24 -h treatment and reduced to $50 \%$ the amount of ENaC- $\alpha$ protein in these cells (135). There was no impact, however, on $\alpha 1$ and $\beta 1 \mathrm{Na}^{+} / \mathrm{K}^{+}$-ATPase mRNA expression (135). Amiloride-sensitive currents and ouabain-sensitive $\mathrm{Rb}^{+}$uptake were reduced. A strong correlation was found at different TNF concentrations between the decrease of amiloride-sensitive current and ENaC- $\alpha$ mRNA expression (135). All these data show that TNF has a profound effect on the capacity of AECs to transport $\mathrm{Na}^{+}$(135). In another study performed by Yamagata et al., mRNA expression of all three $\mathrm{ENaC}$ subunits in whole lung tissue was inhibited by TNF (359). TNF also inhibited $\mathrm{ENaC}$ function, as indicated by the reduction of amiloride-sensitive current (359). These data suggest that TNF may affect the pathophysiology of ALI and pulmonary edema through the inhibition of AFC and sodium transport (359).

\section{TNF Increases Permeability of the Epithelial- Endothelial Barrier}

The activation of TNF receptor 1 by TNF modulates the integrity of the alveolar barrier, in addition to its direct effects on ion channels and pumps of the alveolar epithelium. TNF increases the endothelial expression of chemo-attractants and adhesion molecules including IL-8 (formerly called neutrophil chemotactic factor), the IL-8- receptor 2, the intercellular adhesion molecule-1 (ICAM-1), platelet endothelial cell adhesion molecule-1 (PECAM-1), and vascular adhesion molecule-1, thus promoting excessive recruitment of mononuclear phagocytes and neutrophils during lung inflammation (71, 369-371).

Tumor necrosis factor is released in acute inflammatory lung syndromes linked to the extensive vascular dysfunction associated with increased permeability and EC apoptosis (372). The critical importance of the pulmonary vascular barrier function is shown by the balance between competing EC contractile forces, which generate centripetal tension, and adhesive cell-cell and cell matrix tethering forces, which regulate cell shape. Both competing forces in this model are intimately linked through the endothelial cytoskeleton, a complex network of actin microfilaments, microtubules, and intermediate filaments, which combine to regulate shape change and transduce signals within and between ECs (66).

Tumor necrosis factor can activate ECs, cause acute pulmonary vascular endothelial (VE) injury or even EC death and increase pulmonary vascular permeability in vivo as well as in vitro (39, $67,373)$. Also, TNF increases the permeability of EC monolayers to macromolecules and lower molecular weight solutes by involving pertussis toxin-sensitive regulatory G protein (374). Furthermore, it is reported that TNF can increase the permeability of lung EC monolayers and that fibronectin can blunt this effect (375). In addition, TNF-induced increase in endothelial permeability involves the loss of fibronectin and remodeling of the extracellular matrix (376). Moreover, it has also been shown that TNF can increase capillary permeability causing transcapillary filtration in vivo (377).

\section{TNF Increases ROS Generation}

In addition to the above-mentioned mechanisms, TNF can induce pulmonary edema indirectly through increasing ROS (336). ROS have been shown to be able to disrupt the pulmonary endothelial barrier (336) and to decrease $\mathrm{Na}^{+}$channel activity (378).

\section{Identification of the Alveolar Liquid Clearance-Promoting Effects of TNF Lung Transplantation and Primary Graft Dysfunction (PGD)/Ischemia-Reperfusion Injury}

The receptor-independent lectin-like domain of murine TNF has a potential physiological role in the resolution of alveolar edema in an in situ mouse lung model and an ex vivo rat lung model (99). The lectin-like domain of TNF can activate amiloride-sensitive sodium uptake in type II AECs $(99,100)$. Therefore this TNF domain is a potential therapeutic candidate (360).

As there is no specific treatment for ischemia-reperfusionmediated lung injury, which is accompanied by a disrupted 
capillary barrier integrity and an impeded AFC, the capacity of the TNF tip peptide to improve lung function after unilateral orthotopic lung iso-transplantation was tested in vivo in adult rats (8).

The unilateral rat transplant study showed that a highly severe lung injury with blood gas parameters qualifying for severe ARDS could be virtually prevented by the activation of the TNF lectin-like region. Furthermore, a significant reduction in polymorphonuclear neutrophilic leukocytes (PMN) infiltration in the bronchoalveolar lavage fluid was observed. The TNF tip peptide reduced ROS generation in the transplanted rat lungs in vivo and diminished ROS generation in pulmonary artery ECs in vitro under hypoxia and reoxygenation (8). ROS, the generation of which is increased during ischemia-reperfusion ALI (379-381), have been shown to be able both to disrupt pulmonary endothelial barrier integrity (378) and to inhibit ENaC activity (382).

Moreover, the effect of the lectin-like domain of TNF likely has physiologic relevance during inflammation and infection (8). As the soluble TNF receptors are cleaved by the same enzyme that generates soluble TNF, i.e., TACE (383), complexes between soluble TNF receptors and TNF can form (8). Soluble TNF receptors do not inhibit the activity of the lectin-like domain of TNF and complexes between these receptors and TNF are even able to stimulate AFC in in situ flooded rat lungs $(37,99,353)$. At the same time, unfavorable actions of TNF on edema reabsorption and formation that are mediated by TNF receptor 1 activation are being blocked by the soluble receptors (37). Therefore, the favorable actions of the lectin-like domain of TNF might occur in conditions where both TNF and its soluble receptors are being generated (8).

A recent pilot study of 20 patients on treatment of PGD by twice daily nebulized $125 \mathrm{mg}$ inhalation of the TNF tip peptide (AP301, solnatide) randomized 1:1 showed an improved gas exchange (mean and SD, daily measured up to $72 \mathrm{~h}, \mathrm{PaO} 2 / \mathrm{FiO} 2$ $365.6 \pm 90.4$ versus $335.2 \pm 42.3 \mathrm{~mm} \mathrm{Hg} ; p=0.049)$ and clearly less time intubated $(2 \pm 0.82$ versus $3.7 \pm 1.95$ days, $\mathrm{p}=0.02)$ in the verum group, which also seems clinically relevant (357).

In summary, the lectin-like activity of TNF, and thus, the TNF tip peptide significantly improves lung function after lung transplantation in the rat. Pilot studies confirm a relevant effect in clinical treatment $(8,357)$. The experimental model showed a reduced alveolar neutrophil content and less ROS generation. It exerts a favorable effect on organ function in terms of gas exchange (8). It was furthermore shown that the apically expressed $\mathrm{ENaC}$ was found to be decreased at the messenger ribonucleic acid and the protein level in transplanted lungs, suggesting that $\mathrm{ENaC}$, rather than the basolaterally expressed $\mathrm{Na}^{+} / \mathrm{K}^{+}$-ATPase, is important in the abnormal AFC (101). These studies reinforce the idea that the TNF tip peptide acts as an agent with potential therapeutic traits against the ischemia-reperfusion injury associated with lung transplantation.

\section{The Lectin-Like Region of TNF Ameliorates High-Altitude Pulmonary Edema (HAPE) in Rats}

About 100 million people live at altitudes greater than $2,500 \mathrm{~m}$, about 15 million above $3,000 \mathrm{~m}$, and some above 5,000 $\mathrm{m}$ (384).
Most of these individuals have developed the ability to live and reproduce at elevation as high as $5,000 \mathrm{~m}$, but in some cases, develop chronic medical problems due to their high-altitude residence. At 5,500 $\mathrm{m}$ barometric pressure is about only half of the one at sea level. Furthermore, many lowlanders venture to high altitude for work and recreation. The prevalence of HAPE depends on an individual's susceptibility, the rate of ascent, the final altitude, but also on heavy and prolonged exercise, and is higher in males (385). Although the mechanism underlying HAPE remains incompletely understood, it appears that the elevated pulmonary artery pressure plays a pivotal role in the process. Multiple studies demonstrated that susceptible individuals have abnormally high pulmonary artery pressure in response to hypoxic breathing, during normoxic and hypoxic exercise, and on high altitude before the onset of edema. Increased sympathetic tone, and alteration in vasoactive mediators such as endothelin-1, NO produced by pulmonary ECs, may also lead to stronger hypoxic pulmonary vasoconstriction (384). In autopsies, a red cell rich proteinaceous alveolar exudate with hyaline membrane is characteristic. In all autopsies, areas of pneumonitis with neutrophil accumulation but no evidence of bacterial accumulation have been observed. The estimated death rate of altitude illness is about 7.7/100,000 trekkers, with increasing mortality during the last decade (386). Treatment of HAPE consists, if ever possible, in descent from altitude, rest, oxygen supplementation, and administration of drugs like corticosteroids and furosemide.

Prophylactic inhalation of salmeterol, an inhalative $\beta 2$ adrenergic receptor ( $\beta 2 \mathrm{AR}$ ) agonist, decreased the incidence of HAPE by more than $50 \%$ (387). The most pertinent explanation was that salmeterol would enhance the clearance of alveolar fluid since $\beta 2$-adrenergic agonists upregulate AFC by stimulating transepithelial sodium transport. This hypothesis is supported by the fact that the level of sodium transport in the respiratory epithelium is lower in patients prone to HAPE. However, the study results cannot exclude the possibility that the $\beta 2$ agonist could have modulated vascular permeability or the hemodynamic response associated with hypoxemia and HAPE (4).

In an experimental rat model simulating HAPE by hypobaric and hypoxic conditions equivalent to an altitude of $4,500 \mathrm{~m}$ with exhaustive treadmill exercise of $15 \mathrm{~m}$ per minute for $24 \mathrm{~h}$, then for an equivalent of altitude of $6,000 \mathrm{~m}$ for further $48 \mathrm{~h}$, the TNF tip peptide reduced pulmonary edema and increased expression of the epithelial TJ protein occludin, as compared to high-altitude controls. Compared to untreated high-altitude control animals, TNF tip peptide significantly lowered levels of the inflammatory cytokines TNF, IL-1 $\beta$, IL- 6 and the chemokine IL- 8 in bronchoalveolar lavage. TNF tip peptide-treated animals experienced less pulmonary edema, as compared to dexamethasone-treated animals, and was more effective than its comparators in reduction of bronchoalveolar lavage protein content and inflammatory parameters (7).

\section{Identification of the Mechanism of ENaC Activation by the Lectin-Like Region of TNF}

It has been shown that the lectin-like domain of TNF can activate $\mathrm{ENaC}$ (353) and increases sodium uptake capacity in type II AEC (38). Intriguingly, the TNF tip peptide was shown to directly 
bind to the $\alpha$ subunit of $\operatorname{ENaC}(54,102)$ in a two-hit manner, first interacting with the glycosylated extracellular loop of the subunit and subsequently in the TM 2 domain, where the actual activation of the channel occurs $(54,102,114)$. The former interaction was proposed to increase the expression of $\mathrm{ENaC}$ at the surface membrane in the presence of bacterial toxins, whereas the latter increases the channel's open probability time (102). Indeed, the binding of ENaC to the lectin-like domain of TNF or to the TNF tip peptide stabilizes the channel's complex formation with myristoylated alanine-rich $\mathrm{C}$ kinase substrate and with phosphatidylinositol 4,5-bisphosphate, both of which are important for the open conformation of the channel (388), in the presence of the pneumococcal pore-forming toxin pneumolysin (PLY), an important mediator of permeability edema in pneumococcal pneumonia (54). Knock-in mice expressing a TNF mutant lacking a functional lectin-like domain was shown to be more prone to develop capillary leak and permeability edema than their wildtype counterparts after instillation of a low dose of PLY, which did not induce significant barrier dysfunction in control mice (54). In short, these results demonstrate a novel TNF-mediated mechanism of direct $\mathrm{ENaC}$ activation and indicate a physiological role for the lectin-like domain of TNF in the resolution of alveolar edema during inflammation (54).

\section{The Lectin-Like Region of TNF Increases Activity of $\mathrm{Na}^{+} / \mathrm{K}^{+}$-ATPase}

Vadasz et al. investigated the impact of the TNF tip peptide on fluid balance in experimental lung injury. Alveolar-capillary permeability and fluid clearance were assessed in adult male rabbits. Aerosolized TNF tip peptide improved ALC by both reducing vascular permeability and by enhancing the absorption of excess alveolar fluid in experimental lung injury. TNF tip peptide increased $\mathrm{Na}^{+} / \mathrm{K}^{+}$-ATPase activity by promoting its exocytosis to the AEC surface and increased amiloride-sensitive sodium uptake, which increased the active $\mathrm{Na}^{+}$transport 2.2-fold and consecutively the AFC (196). Together with its previously discussed effects on $\mathrm{ENaC}$, these data suggest a role for the TNF tip peptide as a potential therapeutic agent in pulmonary edema (196), since the two main mediators of $\mathrm{Na}^{+}$transport are both activated by the TNF tip peptide. It should be noted that the primary target is likely $\mathrm{ENaC}$ and that the activation of $\mathrm{Na}^{+} / \mathrm{K}^{+}$-ATPase could be through the indirect increase in intracellular $\mathrm{Na}^{+}$upon prior stimulation of $\mathrm{ENaC}$ (8). Moreover, the TNF tip peptide was recently also shown to increase the activity of NSC channels (9).

\section{The Lectin-Like Region of TNF Restores ENaC Function in PHA1B Mutants}

The lectin-like domain of human TNF activates the ENaC in various cell- and animal-based studies. The synthetically produced cyclic peptides Solnatide (a.k.a. tip peptide or AP301) and its congener AP318 possess molecular structures that mimic the TNF tip region. AP318-mediated $\mathrm{ENaC}$ activation was shown to rescue loss of function in a phenotype of $\mathrm{ENaC}$ carrying mutations and restored the amiloride-sensitive $\mathrm{Na}^{+}$current to physiological levels or even higher (118). This implies that the TNF tip domain can activate $\mathrm{ENaC}$ by a mechanism which remains intact even in the presence of various mutations occurring in different subunits, because binding to the putative binding site in the TM 2 domain of the glycosylated $\alpha$ subunit apparently remains basically unaffected in all tested point mutations or was compensated in frame shift mutations via a moderate activation of $\alpha \beta$ - and $\beta \gamma$-ENaC, respectively (389). Apart from the mechanism responsible for loss of the $\mathrm{ENaC}$ performance in the studied $\mathrm{ENaC}$ mutations, the synthetic TIP and AP318 peptides could restore ENaC function up to or even higher than current levels of wild-type $\mathrm{ENaC}$ (118). As therapy of PHA1B is only symptomatic so far, these TNF tip peptides, which directly target $\mathrm{ENaC}$, are promising candidates for the treatment of the channelopathy-caused disease PHA1B (118).

\section{Clinical Trials on the Effect of the Lectin-Like Region of TNF}

In a recent phase 2 a clinical trial with ALI, patients received inhalable TNF tip peptide in the ventilator twice daily over a 7 -day period. There was no significant improvement in lung liquid clearance over all patients, as assessed by the PiCCO method. However, there was a significant increase in extravascular lung water removal in those patients with a sequential organ failure assessment score higher than or equal to 11, representing more than $50 \%$ of the subjects in this trial (358). One hypothesis for this observation is that patients in this group, apart from suffering from impaired AFC capacity, might also suffer from more severe capillary barrier dysfunction. The TNF tip peptide was recently shown to not only improve AFC (54, 102), but also capillary barrier function (97) in the presence of bacterial toxins.

As mentioned before, in a randomized pilot study performed with 20 patients on the treatment of established PGD after lung transplantation by twice daily inhalation of the TNF tip peptide (AP301, solnatide) versus placebo, the TNF tip peptide improved gas exchange and clearly reduced the intubation-and thus mechanical ventilation-time in a probably clinically relevant manner (357).

\section{TNF-Related Apoptosis-Inducing Ligand (TRAIL)}

TNF-related apoptosis-inducing ligand, a member of the superfamily of TNF ligands, is a homotrimeric type II TM protein with a conserved C-terminal extracellular domain that mediates receptor binding and which can be cleaved by metalloproteinases to generate a soluble mediator (390). TRAIL is produced by several cell types, including immune cells such as macrophages and $\mathrm{T}$ cells and can be induced by both type I and type III Interferons (IFNs), a family of cytokines with fundamental importance in the innate immune response to viral infections $(209,391)$. Macrophages generate both soluble and membrane-bound TRAIL, which operate through distinct receptors on infected and non-infected, neighboring cells (209). TRAIL is a potent activator of cell death in transformed cells and activates cellular stress pathways in epithelial cells, as such finally leading to caspase-dependent or -independent cell death (209). In view of the prominent role of IFNs in antiviral response, IFN-dependent induction of TRAIL is a prominent regulator of 
disease outcome especially in respiratory viral infection, enters into the scene (209). As such, the IFN/TRAIL signaling axis is of potential interest in disease progression and attenuation of tissue injury during respiratory viral infection (209). Here we focused on the role of TRAIL in edema reabsorption and in alveolar epithelial function.

\section{TRAIL Disrupts the Alveolar Epithelial Barrier}

TRAIL plays adverse roles in viral infection (392-394). On the one hand, TRAIL drives infected cells into apoptosis in order to limit virus distribution (209). On the other hand TRAIL can induce functional and structural damage not only in infected cells, but also in bystander cells, such as uninfected cells of the alveolar epithelium $(199,208)$. As such TRAIL can at the same time prevent viral spreading, but also cause lung injury in acute respiratory viral infection (209). Accordingly, in influenza A virus (IAV) infection, TRAIL acts as a detrimental factor contributing to tissue injury and impaired inflammation resolution when released in excessive amounts by recruited immune cells (209). The activation of proapoptotic and pro-necroptotic pathways in respiratory infection can result in a structural disruption of the airway and the alveolar epithelial barrier, which is a major hallmark of respiratory disease and its progression to the ARDS $(395,396)$.

\section{TRAIL Decreases $\mathrm{Na}^{+} / \mathrm{K}^{+}$-ATPase Expression and Impairs AFC}

Peteranderl et al (199). have investigated whether IAV infection alters $\mathrm{Na}^{+} / \mathrm{K}^{+}$-ATPase expression and function in AECs and the ability of the lung to clear edema. IAV infection reduced $\alpha 1$ $\mathrm{Na}^{+} / \mathrm{K}^{+}$-ATPase expression in the plasma membrane of human and murine AECs and in distal lung epithelium of infected mice. Accordingly, the decreased $\mathrm{Na}^{+} / \mathrm{K}^{+}$-ATPase expression impaired AFC in IAV-infected mice. A paracrine cell communication network between infected and non-infected AECs and alveolar macrophages was identified, which led to decreased alveolar epithelial $\mathrm{Na}^{+} / \mathrm{K}^{+}$-ATPase function, thus to AFC inhibition (199). The IAV-induced reduction of $\mathrm{Na}^{+} / \mathrm{K}^{+}$-ATPase was mediated by a host signaling pathway that involved epithelial type I IFN and an IFN-dependent elevation of macrophage TRAIL (199). In non-infected cells within the IAV-infected lung, TRAIL severely compromised the function of the ion channel $\mathrm{Na}^{+} / \mathrm{K}^{+}$-ATPase, which was mediated by induction of the stress kinase AMPK (199) thereby potentially revealing a cross-link to TRAIL-induced autophagic cell stress pathways in bystander cells both in vitro and in vivo (199). The TRAIL-induced and AMPK-mediated downregulation of the $\mathrm{Na}^{+} / \mathrm{K}^{+}$-ATPase, a major driver of vectorial ion and fluid transport from the alveolar airspace toward the interstitium, resulted in a reduced capacity of IAV-infected mice to clear excessive fluid from the alveoli (395). Thus, TRAIL signaling contributes to intensive edema formation, a hallmark of disease in virus-induced ARDS (395). Notably, this effect of TRAIL on $\mathrm{Na}^{+} / \mathrm{K}^{+}$-ATPase expression was induced independently of cell death pathways elicited by caspases, as treatment of cells and mice with a specific caspase- 3 inhibitor diminished apoptosis in AECs but still allowed for the reduction of the $\mathrm{Na}^{+}$/ $\mathrm{K}^{+}$-ATPase (199).

\section{Transforming Growth Factor- $\beta$ (TGF- $\beta$ )}

Transforming growth factor- $\beta$ is a pleiotropic cytokine with a broad regulatory role in the immune system. Three highly homologous isoforms - TGF $\beta 1$, TGF $\beta 2$, and TGF $\beta 3$ - share a receptor complex and signal transduction pathway, but their tissue expression levels are different (397). All are produced as inactive complexes, which must be activated to bind to their receptors (398). Platelets, T lymphocytes, macrophages, ECs, keratinocytes, smooth muscle cells, fibroblasts, i.e., a wide range of cells, can produce TGF (399). Following wounding or inflammation, all these cells are potential sources of TGF- $\beta$ (400). Receptors for TGF- $\beta$ have been found almost on every cell type tested so far, which enables this cytokine to exert its effects on almost any body tissue (401). Classically, TGF- $\beta$ receptor signaling occurs by activating the Smad-dependent intracellular signaling pathway (398). The TGF $\beta$ receptor complex consists of two receptor subunits, TGF- $\beta$ receptor (TGF- $\beta$ R) I and II (398). These receptors mediate multiple responses (401).

\section{TGF $\beta$ Context-Dependent Mode of Action}

Transforming growth factor- $\beta$ action is highly context-dependent and can be influenced by cell type, culture condition, interaction with other signaling pathways, developmental or disease stage in vivo and innate genetic variation among individuals (402). As such, TGF- $\beta$ can be both a pro- and anti-inflammatory cytokine, which affects the growth and proliferation of many cell types (399). During inflammation, TGF- $\beta 1$ is also able to effectively inhibit inflammatory response (403). The action of TGF- $\beta$ following inflammatory responses is characterized by increased production of extracellular matrix components, as well as mesenchymal cell proliferation, migration, and accumulation (404). Pleiotropic nature of TGF- $\beta$ modulates expression of adhesion molecules, provides a chemotactic gradient for leukocytes and other cells participating in an inflammatory response in one hand and, in contrast, inhibits them once they have become activated (405). Also in autoimmunity, TGF $\beta$ represents a double-edged sword (406). It can cause both T-cell growth promotion, as well as immune suppression (406).

\section{Role of TGF- $\beta$ Role in Pulmonary Edema}

Transforming growth factor- $\beta$ has a dual role in pulmonary edema. It can up- or downregulate alveolar ion and fluid transport, through its impact on ion channels/pumps (ENaC, CFTR and $\mathrm{Na}^{+} / \mathrm{K}^{+}$-ATPase) or on the pulmonary barrier. As such, TGF- $\beta$ can decrease the expression of $\mathrm{ENaC}$ through decreasing expression of its $\alpha$ subunit mRNA and protein during bacterial infection (132). During ALI/ARDS, increased TGF- $\beta 1$ activity in the distal airspaces promotes alveolar edema by reducing distal airway epithelial sodium and fluid clearance (132). Moreover, TGF- $\beta$ can induce the internalization of $\beta \mathrm{ENaC}$ from the lung epithelial cell surface and, hence, block the sodium-transporting capacity of AECs (133). In fact, TGF- $\beta$ causes the subsequent activation of phospholipase D1, phosphatidylinositol- 4-phosphate 5 -kinase $1 \alpha$, and NADPH oxidase 4 (Nox4) (133). Nox4 activation moreover results in the production of ROS, which in turn reduces cell surface stability of the $\alpha \beta \gamma \mathrm{ENaC}$ complex and thus leads to edema fluid accumulation (371). Apart from its effects 
on ENaC expression, TGF- $\beta$ can also decrease CFTR expression and function (179) and it, moreover, impairs expression of the $\mathrm{Na}^{+} / \mathrm{K}^{+}$-ATPase $\beta 1$ subunit, resulting in decreased $\mathrm{Na}^{+} / \mathrm{K}^{+}$-ATPase activity in lung epithelial cells $(197,198)$.

Transforming growth factor- $\beta$ decreases lung epithelial barrier function (203-205) in vitro by a mechanism that involves depletion of intracellular glutathione $(206,407)$. The cytokine moreover induces endothelial barrier dysfunction via Smad2dependent p38 activation (235).

The integrin $\alpha v \beta 6$ (408) can activate latent TGF- $\beta$ in the lungs and skin (409). Using this clue, Pittet et al. have shown that mice lacking integrin $\alpha v \beta 6$ are completely protected from pulmonary edema in bleomycin-induced ALI. Furthermore, pharmacologic inhibition of TGF- $\beta$ also protected wild-type mice from pulmonary edema induced by bleomycin or Escherichia coli endotoxin (206). In short, integrin-mediated local activation of TGF- $\beta$ is critical for the development of pulmonary edema in ALI, and blocking TGF- $\beta$ or its activation attenuates pulmonary edema. This neutralization can be done e.g., by the administration of a soluble type II TGF- $\beta$ receptor, which sequesters free TGF- $\beta$ during lung injury (206).

All of the deleterious actions of TGF- $\beta$ discussed above will ultimately lead to decreased ion transport and may, therefore, promote and worsen pulmonary edema. However, TGF- $\beta$ can also positively impact pulmonary edema. Intriguingly, TGF- $\beta$ was proposed to increase the function of $\mathrm{ENaC}$, via enhancing the expression of $\mathrm{Na}^{+} / \mathrm{K}^{+}$-ATPase $\alpha 1$ - and $\beta 1$-subunits (134).

\section{Interleukin-8}

Interleukin- 8 is a pro-inflammatory chemokine produced by a variety of tissue and blood cells (410), including bronchial epithelial cells (411), that correlates with neutrophil accumulation in distal airspaces of patients with ARDS. IL-8 is also a predictor of mortality in ALI (412-414). As such, significantly higher concentrations of IL-8 are found in the pulmonary edema fluid and plasma of patients with a septic versus a non-septic etiology of ARDS (415). Moreover, IL-8 promotes edema formation by blocking AFC (105).

\section{The Role of IL-8 in Inhibiting $\beta 2$ AR Agonist}

Roux et al (105). have shown that IL-8 or its rat analog cytokineinduced neutrophil chemokine-1 significantly decreased $\beta 2 \mathrm{AR}$ agonist-stimulated vectorial $\mathrm{Cl}^{-}$and net fluid transport across rat and human alveolar epithelial type II cells, through reducing CFTR activity and biosynthesis (105). This reduction process was mediated by heterologous $\beta 2 \mathrm{AR}$ desensitization and downregulation (50\%) via the G-protein-coupled receptor kinase 2 (GRK2)/ PI3K signaling pathway (105) (Figure 8). Consistent with the experimental results, high pulmonary edema fluid levels of IL-8 $(>4,000 \mathrm{pg} / \mathrm{ml})$ were associated with impaired AFC in patients with ALI. Taken together, these results suggest a role for IL-8 in inhibiting $\beta 2 \mathrm{AR}$ agonist-stimulated alveolar epithelial fluid transport via a GRK2/PI3K-dependent mechanism (105). On top of this, IL- 8 can promote edema formation by increasing endothelial permeability (250).



FIGURE 8 | Rationale for the problematic role of $\beta 2$ adrenergic agonists in clinical trials. Schematic representation of the mechanisms by which interleukin-8 (IL-8)/ cytokine-induced neutrophil chemokine (CINC)- 1 and transforming growth factor (TGF)- $\beta 1$ have a synergistic inhibitory effect on the $\beta 2$-adrenergic receptor ( $\beta 2 A R$ ) signaling pathway in type II alveolar (ATII) cells. IL-8/CINC-1 and TGF- $\beta 1$ cause the activation of different phosphoinositide 3-kinase (PI3K) isoforms. However, IL-8/ CINC-1 but not TGF- $\beta 1$ phosphorylates G-protein-coupled receptor kinase 2 (GRK2) via a protein kinase C-zeta (PKC- $\zeta$ )-dependent mechanism explaining why the blockade of IL-8/CINC-1 prevents the TGF- $\beta 1$-mediated inhibition of the $\beta 2 A R$ signaling pathway in ATII cells. This results in the translocation of the protein complex GRK2 and PI3K to the cell membrane. This protein complex causes phosphorylation at the Ser355 heterologous desensitization and downregulation of the $\beta 2 A R$ in ATII cells. IL-8/CINC-1 and TGF- $\beta 1$ then prevent the activation of $3^{\prime}-5^{\prime}$-cyclic adenosine monophosphate (cAMP)/protein kinase A (PKA) pathway that upregulates the vectorial fluid transport across the alveolar epithelium via phosphorylation and increased expression of cystic fibrosis transmembrane conductance regulator (CFTR) at the plasma membrane of ATII cells. The solid lines indicate the pathways stimulated by IL-8/CINC-1 and the dashed lines indicate the pathways inhibited by these mediators (416). 


\section{Interleukin-1 $\beta$}

Interleukin- $1 \beta$ is associated with decreased alveolar fluid reabsorption and thus with worse outcome in ALI and sepsis. IL-1 $\beta$ primarily decreases alveolar fluid reabsorption via a p38 MAPK, reducing the expression of the $\alpha$-subunit of ENaC (113) as well as the $\beta$-subunit (137). In ARDS patients, the mean initial plasma levels of TNF IL-1 $\beta$, IL-6, and IL- 8 were significantly higher in non-survivors and in patients with sepsis. High plasma levels of IL- $1 \beta$ were associated with poor patient outcome (417). Likewise, high levels of IL-1 $\beta$ in the lungs of patients with ARDS were associated with an increased risk of mortality (417). The FAS/CD95 system acts together with TNF and IL- $1 \beta(57,219,418-420)$, leading to NF-kB production and neutrophil accumulating IL-8 secretion. Of note, an epithelial repair effect for type II pneumocytes via IL-1 $\beta$ was described in the injured alveolus (139), possibly in a specific context of cytokines, mediators and growth factors (139). Only in a specific fetal context IL-1 $\beta$ may increase alveolar fluid reabsorption by a hypothalamus-pituitary-adrenal gland axis (421) and an increase of both $\mathrm{ENaC}$ and $\mathrm{Na}^{+} / \mathrm{K}^{+}$-ATPase expression (140).

\section{Fas/FasL System (CD95/CD95 Ligand System)}

Fas is a $45-\mathrm{kDa}$ type I cell surface receptor that belongs to the TNF receptor family. It can cause cytokine and chemokine release, especially the neutrophil attractant IL-8, via MAP kinase activation in lung epithelial cells, as such promoting inflammation (219). Binding of FasL to membrane Fas activates apoptosis through activation of caspases, which seems the key to AEC apoptosis, thus epithelial barrier breakdown and its consequences in $\operatorname{ALI}(57,418)$.

\section{Keratinocyte Growth Factor (KGF, FGF-7)}

KGF is an epithelial cell-specific growth factor that has been shown to exert beneficial actions in many animal models of ALI and ARDS as well as in the ex vivo human lung (143, 422-430). Rats in which KGF was intratracheally administered increased AFC by about up to $50 \%$, and this was further increased by the $\beta 2$ agonist terbutaline (427). In vitro studies using mesenchymal stem cell-derived medium suggested that this growth factor plays a dominant role in tissue repair, even in the presence of the inflammatory cytokines IL- $1 \beta$, TNF- $\alpha$, and interferon-gamma, as well as in hypoxia. The observation that no downregulation of $\mathrm{ENaC}-\alpha$ expression occurred despite of the presence of three key inflammatory cytokines suggested a dominant biological role of KGF in the acutely injured alveolar milieu (431). There is a currently a large interest in stem cell therapies as therapeutic approaches in clinical disorders like myocardial infarction, limb ischemia, diabetes, hepatic and renal failure, and ALI/ARDS. Stem and progenitor cell therapies as well as work with factors influencing those cells to reduce injury and increase repair have been performed. KGF has been proposed to be one of the main candidates to promote the repair capacity of stem cells in ALI. A recently performed double-blind, placebo-controlled phase 2 clinical trial-the
KARE trial-tested the effects of KGF in 29 verum patients versus 31 placebo patients (432). There was no difference in the primary outcome variable, the oxygenation index, at day 7 , and the treatment group had a trend to higher mortality, and more adverse events in terms of pyrexia. Nevertheless, these data do not exclude that the combined use of KGF and stem cells might provide protection in ALI.

\section{Soluble Receptor for Advanced Glycation End Products (sRAGE)}

Receptor for advanced glycation end products, first characterized in 1992 by Neeper et al. is a $35 \mathrm{kDa}$ TM receptor which belongs to the immunoglobulin superfamily (433). RAGE is one of the AT1 cell-associated proteins in the lungs $(434,435)$. RAGE and its ligands have been recognized to be involved in the pathobiology of a wide range of diseases which are accompanied by symptoms, like enhanced oxidative stress, immune/ inflammatory responses, and altered cell functions (436). RAGE is highly expressed in the lungs at readily measurable levels and its level increases quickly at sites of inflammation, mainly in inflammatory and epithelial cells (437). RAGE has three forms, consisting of $\mathrm{N}$-truncated, dominant-negative, and soluble RAGE, which can be produced either by natural alternative splicing or by the action of membrane-associated proteases (438). The correlation between sRAGE levels and AFC rate was investigated in both a clinical study of patients with ARDS, as well as in an experimental model of acid-induced lung injury in mice (264). The results obtained showed a correlation between elevated levels of sRAGE with lung injury and an impairment of AFC (264). Accordingly, an increase in alveolar-capillary barrier permeability, arterial oxygenation impairment, lung injury scores, and the extent of human lung damage on CT scan are all associated with sRAGE levels (264). Conversely, it has been shown that RAGE regulates lung fluid balance via protein kinase C-gp91(phox) signaling to $\mathrm{ENaC}$ (177). In fact, hAGE, a RAGE ligand, increases $\mathrm{ENaC}$ activity through oxidant-mediated signaling, which can ultimately impact lung fluid clearance (177).

\section{B2ARs AS IMPORTANT MODULATORS OF AFC}

\section{Structure and Subtypes}

$\beta 2$-adrenergic receptors are $G$ protein-coupled receptors with seven-TM domains (439). Their three subtypes are $\beta 1$, predominantly found in the heart, $\beta 2$ in the respiratory system, and $\beta 3$ in adipose tissue (440). $\beta 2$ adrenergic agonists activate the $\beta 2$ adrenoceptors $(\beta 2 \mathrm{AR})$ on airway smooth muscle and are used to treat bronchoconstriction in asthma and chronic obstructive pulmonary disease (COPD) (441). In their canonical signaling pathway, agonist binding couples the $\beta 2 \mathrm{AR}$ to the Gs subtype of $\mathrm{G}$ protein. Gs activation leads to adenylyl cyclase, production of cAMP and activation of the cAMP-dependent protein kinase A (PKA), which mediates most of the functional consequences of Gs-coupled receptor activation (442). In airway smooth muscle, $\beta 2 \mathrm{AR}$-stimulated PKA activity mediates relaxation through 
phosphorylation of multiple proteins involved in regulating intracellular calcium levels, calcium sensitivity, and cross-bridge cycling (442).

\section{The Role of $\beta 2 A R$ Agonists in AFC}

The presence of pulmonary $\beta 2 \mathrm{ARs}$ includes the alveolar space and provides the possibility to modulate the active $\mathrm{Na}^{+}$transport. $\beta 2$ adrenoceptors and the $\beta$-adrenergic agonists accelerate AFC (439) due to $\mathrm{Na}^{+}$transport via an amiloride-sensitive pathway (443) as shown in vitro (444), ex vivo (445), and in vivo in rat (446), dog (447), sheep (448), guinea pig (449), mouse (443, 450), and human lung tissue (451). $\beta 2 \mathrm{AR}$ knockout mice results suggest that the $\beta 2 \mathrm{AR}$ is responsible for most of the $\beta$-adrenergic-mediated upregulation of AFC (452). Therefore, $\beta 2$ ARs appear to be responsible for the bulk of the $\beta$-receptor-sensitive alveolar active $\mathrm{Na}^{+}$transport likely due to direct and indirect up-regulation of the alveolar active $\mathrm{Na}^{+}$transport $(445,449,452-454)$. $\beta$-agonists via activation of $\beta 2 \mathrm{ARs}$ regulate necessary key proteins for the process of alveolar epithelial active $\mathrm{Na}^{+}$transport such as $\mathrm{ENaC}$, $\mathrm{Na}^{+} / \mathrm{K}^{+}$-ATPase and CFTR in animal models as well as in human lung tissue $(445,449,453,455)$. $\beta 2 \mathrm{ARs}$ mediate short-term regulation of $\mathrm{Na}^{+}$pumps which occurs within minutes of receptor engagement via highly regulated recruitment of assembled $\mathrm{Na}^{+} / \mathrm{K}^{+}$-ATPase from intracellular compartments through phosphorylation of intermediary proteins and RhoA-kinase $(456,457)$. Long-term regulation is carried out via transcription (458) and translation of $\alpha 1$-subunit of $\mathrm{Na}^{+} / \mathrm{K}^{+}$-ATPase and $\mathrm{ENaC}$ subunits through PKA induced phosphorylation of cAMP-responsive elements and post-transcriptional regulation via mitogen-activated protein kinase/extracellular signal-regulated kinase and rapamycin sensitive pathways $(455,459)$ by direct modulation of $\mathrm{Na}^{+}$ channels at the apical surface of the cells (460) or an activation of PKA to modulate a cation channel $(92,453)$.

\section{Impact of $\beta 2 A R$ Agonists on ENaC}

Protein kinase A-mediated $\beta 2$-agonist action phosphorylates cytoskeleton proteins and promotes trafficking of $\mathrm{Na}^{+}$channels through the cell membrane and direct phosphorylation of epithelial $\mathrm{Na}^{+}$channel $\beta$ and $\gamma$ subunits stimulate the $\beta 2 \mathrm{AR}$ and increases the number of epithelial $\mathrm{Na}^{+}$channels and their open time in alveolar type II cells (453) and enhances the expression of the $\alpha$-subunit of the epithelial $\mathrm{Na}^{+}$channel $\mathrm{ENaC}$ (458). $\beta$-agonists and cAMP analogs increase the open probability and open time of amiloride-sensitive $\mathrm{Na}^{+}$channels (161). $\beta 2 \mathrm{AR}$ agonists thus increase $\mathrm{Na}^{+}$flux across the apical cell membrane by increasing both membrane-bound channel abundance and $\mathrm{Na}^{+}$flux through $\mathrm{ENaC}(439)$.

\section{Impact of $\beta 2 A R$ Agonists on $\mathrm{Na}^{+} / \mathrm{K}^{+}$-ATPase}

$\beta$-adrenergic agonist modulate $\mathrm{Na}^{+} / \mathrm{K}^{+}$-ATPase partially through adenosine 3',5'-cyclic monophosphate (461). $\beta 2$-adrenergic agonists increase the gene expression of $\mathrm{Na}^{+} / \mathrm{K}^{+}$-ATPase which leads to:

- Increased expression of $\alpha 1-\mathrm{Na}^{+} / \mathrm{K}^{+}$-ATPase mRNA and protein (458).

- Increase of the quantity of $\mathrm{Na}^{+} / \mathrm{K}^{+}$-ATPase (458)

- Increased activity of $\mathrm{Na}^{+} / \mathrm{K}^{+}$-ATPase $(456,458,462-464)$.

\section{Impact of $\beta 2 A R$ Agonists on CFTR}

Cystic fibrosis transmembrane conductance regulator is required for cAMP-mediated upregulation of fluid clearance, but is not necessary for basal fluid absorption (183), thus for alveolar fluid homeostasis in the uninjured lung $(182,183) . \beta 2$-adrenergic stimulation activates CFTR by cAMP and PKA activation (184). In airway epithelial cells, the interaction of $\beta 2$-AR with CFTR is mediated by scaffold proteins, such as NHERF1, allowing its interaction with PKA and stabilizing it on the plasma membrane (465). $\beta 2$-adrenergic stimulation increases CFTR regulator expression in human airway epithelial cells through a cAMP/ PKA-independent pathway (466).

\section{B2-Adrenergic Agonists Are at Least in Part Not of Clinical Benefit in ALI/ARDS Studies and May Increase Mortality}

In mild-to-moderate lung injury, alveolar edema fluid clearance is often preserved by catecholamine-dependent or -independent mechanisms (467). Stimulation of AFC is then related to activation or increased expression of sodium channels like $\mathrm{ENaC}$ or the $\mathrm{Na}^{+} / \mathrm{K}^{+}$-ATPase pump and may involve CFTR (467). In severelung injury, AFC perturbation result through increased endothelialinterstitial-epithelial alveolar permeability and changes in activity or expression of sodium or chloride transport molecules (467). Improved barrier function and increased alveolar fluid reabsorption, theoretically by $\beta$-adrenergic agonists or the lectin-like TNF activity or alternatives, vasoactive drugs, regenerative or repair measures are therefore therapeutic alternatives (467). Whereas in the BALTI-2 study with salbutamol given as an intravenous infusion for up to 7 days, compared with a placebo, more than 160 patients [age 55 (SD 17) years] per group were studied, the study was stopped as salbutamol treatment was associated with increased 28 -day mortality of $34 \%$ compared to $23 \%$ (risk ratio $1.47,95 \%$ confidence interval 1.03 to 2.08 ) (468).

Salbutamol early in the course of ARDS was poorly tolerated. The authors concluded that such a $\beta 2$-agonist therapy is unlikely to be beneficial and could worsen outcomes. Follow-up data further suggested worse outcome at 6 and 12 months in ARDS patients treated with salbutamol. They discussed that further trials of $\beta$-agonists in patients with ARDS were therefore unlikely to be conducted.

Some questions remained open, such as whether or not there may be benefit at a different dose or in specific populations (468). The survival curves for salbutamol and placebo appeared to continue to diverge after the end of the study drug infusion after 7 days, suggesting that the mechanisms may involve indirect effects as, e.g., more systemic disease under and after intravenous salbutamol. Concerning morbidity and mortality, Salbutamol can cause arrhythmia and tachycardia, and electrolyte and metabolic disturbances such as hypokalemia, hypomagnesemia, and lactic acidosis, which was observed in the study, and led to more salbutamol discontinuation. The used salbutamol dose of $15 \mu \mathrm{g} / \mathrm{kg}$ ideal body weight/hour i.v. was considered the maximum that critically ill patients could receive without an increase in ventricular or atrial tachycardia or ectopy. It was at the higher end of the recommended dosing regimen, and it is possible that lower 
doses might have been better tolerated and caused fewer adverse outcomes (468).

Rather similar results were observed in the USA in the ALTA trial (Albuterol for the Treatment of ALI). ALTA was a placebocontrolled multicentre study of nebulized salbutamol in patients with ALI. Patients were randomized to receive either salbutamol $5 \mathrm{mg}$ every $4 \mathrm{~h}$ or saline placebo, for up to 10 days. The primary outcome was ventilator-free days. Recruitment started 2007 with a target sample size of 1,000 patients. It was terminated after 282 patients had been enrolled because of futility. There was no clear difference observed in both ventilator-free days between the salbutamol and placebo arms (14.4 versus 16.6 days; $95 \%$ CI -4.7 to 0.3 days) or in hospital mortality (salbutamol $23.0 \%$ versus placebo $17.7 \%$; $95 \% \mathrm{CI}-4.0 \%$ to $14.7 \%)$. Although the $\beta 2$ stimulator intervention was delivered by a different route in ALTA, and the early termination of recruitment caused that confidence intervals are wide, the results seemed much consistent with the BALTI-2 trial.

One alternative way was to use combination of inhaled corticosteroid and inhaled $\beta 2$ agonist. In a recently published pilot study, a typical asthma treatment combination of twice daily inhaled formoterol and budesonide for 5 days showed its feasibility and promising results. The rationale was to reduce by both budesonide and formoterol alveolar inflammation, and to further improve by formoterol AFC. The aim was to reduce ARDS. More patients in the placebo group developed ARDS (7 versus 0 ) and required mechanical ventilation (53\% versus $21 \%$ ) (469).

\section{Further Potentially Critical Mechanisms of Action $\beta$-Adrenergic Agonists}

Besides two futile ARDS trials, further factors might restrict the $\beta 2$ receptor agonist usage as a therapy to increase the resolution of pulmonary edema (467). Prolonged stimulation of $\beta$-adrenergic receptors with endogenous catecholamines could desensitize the $\beta$-receptors and prevent their stimulation with exogenous catecholamines (467). For instance, in some patients the alveolar epithelium might be too injured to respond to $\beta$-adrenergic agonist therapy (467), likewise circulating factors could limit the action of $\beta$-adrenergic agonists (467). Also, in the presence of left atrial hypertension, atrial natriuretic peptide can inhibit the stimulatory effect (467). Similarly in prolonged hemorrhagic shock and resuscitation, cAMP agonists may not stimulate AFC because oxidant-mediated injury may reduce the response of the alveolar epithelium to $\beta$-2 agonists (467).

An important clinical aspect is the potential to increase cardiac index by $\beta 2$ receptor agonists (470), by both cardiac stimulation and pulmonary arterial vasodilation. Cardiac stimulation can lead to a higher cardiac index. This is potentially dangerous, as due to the injured lung put in the circulation in series, there is an increase in filtration, which further increases alveolar fluid and gas exchange disturbance. An interrelated second, and in ALI most probably untoward "Robin Hood effect" of potential opening of vascular beds that are closed by vasoconstriction is, e.g., observed in COPD patients inhaling $\beta 2$ receptor agonists and developing more hypoxemia (471). This is probably due to increased perfusion in badly ventilated ALI/ARDS alveolar areas. As shown by Briot et al., $\beta 2$ receptor agonist therapy seems therefore to have the potential to heighten the protein leakage from plasma to alveoli in the acutely injured lung (470).

\section{PROTEIN CLEARANCE OUT OF THE ALVEOLAR SPACE}

Clearance of serum and inflammatory proteins from the alveolar space is an important and possibly vital process in recovery from pulmonary edema. Albumin and IgG are present in pulmonary edema fluid in concentrations that are $40-65 \%$ of plasma levels in hydrostatic pulmonary edema and 75-95\% in non-cardiogenic pulmonary edema. Concentrations of albumin, for example, may be $5 \mathrm{~g} / 100 \mathrm{ml}$ or more. Protein concentrations rise during recovery from alveolar edema because the salt and water fraction of edema fluid is cleared much faster than albumin and IgG. Clearance of alveolar protein occurs by paracellular pathways in the setting of pulmonary edema. Transcytosis may be important in regulating the alveolar milieu under nonpathological circumstances. Alveolar protein degradation may become important in long-term protein clearance, clearance of insoluble proteins, or under pathological conditions such as immune reactions or ALI.

Early since the first descriptions of ARDS, we know that protein content is high, "haemorrhagic," and about the same as plasma proteins. Plasma and coagulative products such as fibrin strands are degraded or modified, e.g., also to hyaline membranes in a high number of patients (31). They are observed in ARDS, are especially covering denuded basement membranes where pneumocytes are missing, and may be related to adverse outcome (56).

Recent research hints to a better understanding of the resolution of those alveolar proteinaceous contents and debris out of the distal airways. Counterintuitively, neither macrophages, nor the mucociliary transport processes seem to play major roles in protein clearance also over several days time (472). Protein clearance from the distal air spaces is in part facilitated by active endocytotic processes including for albumin by the $600 \mathrm{kDa}$ TM glycoprotein called megalin or LDL-receptor related protein-2, a member of the low-density lipoprotein-receptor superfamily (6). Again, its important functional inhibition seems TGF-beta1 related. Megalin seems negatively regulated by glycogen synthase kinase $3 b$ (GSK3b). An important regulator for this protein kinase signaling molecule seems the RNA binding protein Embryonic Lethal, Abnormal Vision, Drosophila Like 1/Human antigen $\mathrm{R}$ (ELAVL-1/HuR) as an upstream regulator of GSK3b (6). ELAVL-1/HuR is an RNA binding protein that increases mRNA stability. Its importance has been shown in ventilatorinduced and acid-induced mouse lung injury. In EC lines it induces ICAM-1 and IL-8 after TNF stimulation.

Endocytosis of macromolecules can be mediated by a nonselective fluid phase uptake, which is a very slow process in alveolar epithelium. A receptor-mediated endocytosis is much faster and occurs when specific high-affinity receptors are implicated. Two pathways are described, called caveolae-mediated and clathrin-mediated endocytosis.

Detailed research on alveolar protein and debris clearance have only recently begun. Judging their roles is more complex, as hyperosmotic stimuli might be of anti-inflammatory action, 
and possibly there is even more biological signaling as formerly assumed that may influence underlying lung disease.

\section{POTENTIAL NOVEL APPROACHES TO UNDERSTANDING THE EFFECTS OF ION CHANNEL STIMULANTS IN LUNG DISEASE}

\section{Hyperosmolarity, High $\mathrm{Na}^{+}$Content, or High Oncotic Pressure}

One biological effect that has, to our knowledge, not yet been assessed is the question whether due to fluid reabsorption out of the alveolus the hyperosmolarity or hyper-oncotic situation is of biological effect. Several limitations have to be mentioned: Certainly the pulmonary surfaces including the mucus and the surfactant system and its layers are complex and disease-prone systems, as suggested in cystic fibrosis. Dose- and time response have to be taken into account. Actually, there are contradictory results on those effects: Some observations described antiinflammatory effects of hyperosmolarity in the airways, as in the nose and sinuses with a few randomized controlled trials that compared isoosmotic versus hyperosmotic irrigating solutions $(473,474)$. Honey is hyperosmotic and antibacterial, and in wound healing it seems frequently beneficial (475). This is also the case for hyperosmotic salt pastilles in throat and neck infections. However, nebulized hypertonic saline is still disputed in infants with acute viral bronchiolitis (476). There are also in vitro cell model results showing a switch from adaptive to inflammatory gene expression by hyperosmotic stress by protein kinase R activation, NF-kappaB p65 activation with responsive genes including inducible NO synthase, interleukin-6, and interleukin-1 $\beta$ (477), others with some protection via p53 gene regulation (478). In a rat seawater drowning model, alveolar hypertonicity, but not iso-or hypotonicity-induced inflammation and vascular leak, thus edema probably by hypoxia-inducible factor- 1 and including ataxia telangiectasia mutated kinase and PI3 kinase (479).

Local $\mathrm{Na}^{+}$accumulation and enhanced availability have been linked to activation of tonicity-responsive enhancer binding protein (TonEBP) via the mononuclear phagocyte system in the skin (480), a system also widely represented in the lung. Enhanced local $\mathrm{Na}^{+}$has been shown to boost pro-inflammatory TH17 cell production and, finally, IL-17 release (481). The pro-inflammatory phenotype is maintained in high-salt conditions with upregulation of TNF- $\alpha$ and IL-2. As it is currently unclear what is the mechanism of enhanced $\mathrm{Na}^{+}$presentation to activate the TonEBP, an enhanced $\mathrm{Na}^{+}$accumulation in the extracellular matrix (482), the activation of $\mathrm{Na}^{+}$channels or even a permissive role of an altered $\mathrm{Na}^{+} / \mathrm{K}^{+}$-ATPase activity via endogenous ouabain have to be considered (483). As all of these mechanisms are also represented in the lung, both $\mathrm{Na}^{+}$ presentation and availability should, therefore, be considered in pulmonary fluid regulation.

Briefly, there may be important, but so far not yet well understood anti-, or even pro-inflammatory, stimuli, or signals by hyperosmotic stimulation, underlining the importance to investigate this subject further.

\section{SPECIFIC CLINICAL SETTINGS WITH POTENTIAL SIGNIFICANCE OF ALVEOLAR FLUID REABSORPTION IN INFLAMED LUNGS}

\section{RDS in the Newborn}

Respiratory distress syndrome is one of the most important causes of morbidity and mortality in newborns and has a prevalence of about $1 \%$. It is clinically manifesting as respiratory distress accompanied by abnormal pulmonary function and hypoxemia directly in the first minutes or hours after birth. RDS prevalence increases with decreasing gestational age. As such the incidence of RDS is highest in extremely preterm infants, affecting more than $90 \%$ of infants at a gestational age of 28 weeks or less. In a birth cohort of more than 230,000 deliveries, the syndrome was observed at 34 weeks gestation in $10.5 \%$, at 35 weeks in $6 \%$, at 36 weeks in $2.8 \%$, at 37 weeks in $1 \%$, at 38 , and more in $0.3 \%$. Therapy is supportive, includes surfactant replacement, fluid restriction, and glucocorticoids. Whereas a viewpoint has been that qualitative and quantitative surfactant deficiency, inflammation including alveolar neutrophil influx, and fluid overload (in part by low urine output) account for this syndrome, some reports hint to a suboptimal $\mathrm{Na}^{+}$transport. During gestation, the lung epithelium secretes $\mathrm{Cl}^{-}$and fluid and develops the ability to actively reabsorb $\mathrm{Na}^{+}$only during late gestation. At birth, the mature lung switches from active $\mathrm{Cl}^{-}$and consecutive fluid secretion to active $\mathrm{Na}^{+}$and consecutive fluid absorption in response to circulating catecholamines. Changes in oxygen tension augment the $\mathrm{Na}^{+}$-uptake capacity of the epithelium and increase $\mathrm{ENaC}$ gene expression. The inability of the immature fetal lung to switch from fluid secretion to fluid absorption results, at least in large part, from an immaturity in terms of low expression of $\mathrm{ENaC}$, where all three $\mathrm{ENaC}$ subunits are low in preterm relative to full-term infants. $\mathrm{ENaC}-\alpha$ is increased in the respiratory epithelium by therapeutic glucocorticosteroids $(484,485)$.

However, in the last years the incidence of near-term and term infants with RDS has increased, and their clinical characteristics differ from those of premature infants with RDS. Li et al. found that death was virtually inevitable for some babies, despite intensive care and surfactant replacement therapy, particularly in near-term and term infants. Lung tissue slices taken during autopsies of near-term and term infants who died of neonatal RDS showed that some alveoli were obviously dilated, with a large amount of lung fluid. This was in addition to an alveolar collapse from a lack of surfactant, and suggested that lung fluid absorption disorders might be an important additional cause of RDS by influencing gas exchange or surfactant function (486). In their study on 120 neonates with RDS and 129 controls, 7 newborns died despite of intensive care and surfactant replacement therapy. All of them received surfactant more than once and four of them were near-term or term infants. Preterm babies (less than 35 weeks of gestational age) had a better response to surfactant treatment than near-term and term babies. These results were consistent with the finding that the surfactant therapy was not effective for all newborns with RDS. The authors assessed the 
relationship between RDS and 7 candidate polymorphisms of the SCNN1A gene that encodes $\alpha$-ENaC. One single nucleotide polymorphism (rs4149570) of the SCNN1A gene was associated with RDS. Moreover, in a group of term infants (gestational age was 37 weeks or greater), another single nucleotide polymorphism locus (rs7956915) was associated with RDS. These results are consistent with the hypothesis that the causes of RDS are multifactorial, and that in term infants it might differ from those in preterm infants (487). Alveolar fluid reabsorption and, thus, $\alpha$-ENaC might play a key role in the pathogenesis by influencing the amount of lung liquid absorption, especially in term infants with RDS.

\section{Acute Infection-Related Respiratory Failure}

Pulmonary infections are the most prevalent infections worldwide, most of bacterial or viral origin. Community-acquired pneumonia is a frequent infectious respiratory disease with an annual incidence of about 5-12/1,000, and leads to hospitalization in $20-50 \%$ of patients. Mortality in hospitalized patients ranges from 5 to $15 \%$. The most common reason for hospital admission in childhood is pneumonia and accounts for up to $50 \%$ of admissions. The high morbidity, mortality, and epidemiologic dangers with viral or bacterial pneumonias are of high concern. Pneumonia mortality is typically caused by flooding of the pulmonary alveoli preventing normal gas exchange and consequent hypoxemia. We refer to excellent recent reviews $(117,371)$. Of note is that pneumonia and sepsis are by far the leading causes of ALI and ARDS. Sepsis is a major healthcare burden, mirrored by up to $45 \%$ of intensive care unit costs (64) and bearing a high mortality of about $30 \%$. Cytokines and ion channels are key elements in this common health problem.

\section{Lung Transplantation}

Lung transplantation is a substitutive treatment of various endstage pulmonary disease. Cystic fibrosis, COPD, and idiopathic pulmonary fibrosis (IPF) are the most important transplanted patient groups (488). The high mortality rate relative to other solid-organ transplants is in part due to chronic rejection. The limited availability of donor lungs results in a highly limited treatment strategy for patients in whom a survival benefit-estimated 5 -year survival is about 60\%-is expected (488).

Primary graft dysfunction (PGD) is termed the development of allograft infiltrates within $72 \mathrm{~h}$ of transplantation together with impaired oxygenation, when other identifiable insults such as volume overload, pneumonia, acute rejection, atelectasis or vascular compromises are excluded. PGD is usually referred to ischemia-reperfusion injury, but additionally to any further mechanical, surgical or chemical trauma such as inflammatory, neural or hormonal events of the donor, high oxygen fraction during reperfusion, or lymphatic disruption. PGD is mild and transient in most cases, but $10-20 \%$ of patient situations are sufficiently severe to cause life-threatening hypoxemia similar to ARDS, based on the same mediators and cytokines and a diffuse alveolar damage resembling ARDS. Similar to ARDS, it is considered a systemic disease, not only affecting the lung, but the whole patient. Thereby, the increased occurrence of cerebral dysfunction, i.e., patient delirium, worsens prognosis. Severe PGD quadruples perioperative mortality, the leading cause of early death of lung transplant recipients. In one study it is associated with a 30 day mortality of 63 versus $9 \%$, and associated duration of mechanical ventilation is 15 versus 1 day (489) (Figures 9 and 10). The risk of higher morbidity and death risk persists even after an often protracted recovery, suggesting that PGD triggers an increased risk for bronchiolitis obliterans syndrome as a manifestation of chronic allograft rejection (490).

Ischemia-reperfusion injury is the main mechanism for PGD (503, 504). With logarithmic function ischemia time is associated with reperfusion injury: Whereas $4 \mathrm{~h}$ ischemia is associated with about $13 \%$ more risk than $2 \mathrm{~h}, 6 \mathrm{~h}$ ischemia increases the risk by more than $50 \%, 8 \mathrm{~h}$ by a factor of 3 , and $10 \mathrm{~h}$ by a factor of about 8 (494). The hypothermic preservation increases oxidative stress, leads to accumulation of intracellular sodium and loss of intracellular potassium and an intracellular calcium overload, cell death with apoptosis (240) and necrosis. The release of pro-and anti-inflammatory cytokines such as TNF, INF- $\gamma$, IL-8, IL-10, IL-12, and IL-18 and complement cause smooth muscle contraction and increase vascular permeability, amplify by C5a the inflammatory response and are chemoattractant. Soluble complement receptor-1 is an accepted, but underused treatment based on a placebo-controlled clinical trial with 59 patients (505).

A huge part of the ischemia-reperfusion injury of lung allografts is mediated by the change in vascular shear stress due to the blood flow cessation. The endothelial sensing mechanism called mechanosome chiefly consists of PECAM-1, VEGR receptor-2 (VEGFR2) and VE cadherin in the EC caveolae (499). It closes the $\mathrm{K}_{\mathrm{ATP}}$ channel of the EC membrane, depolarizes it and leads to NADPH oxidase 2 activation as the main source to generate ROS. EC depolarization results in opening of T-type voltagegated $\mathrm{Ca}^{2+}$ channels, increase intracellular calcium, and $\mathrm{NO}$ synthase activation and consecutive NO-mediated vasodilation, and an overproduction of ROS that causes oxidative injury which triggers inflammation or even cell death (499). PI3K-Akt leads to NADPH activation, producing ROS. With ischemia, there is also an NO production by endothelial NO synthase, probably as a physiological response to the loss of blood flow. The ROS generated in ECs interact with signaling-related proteins and thus with enzymatic activity. NF-kB, activator protein 1 (AP-1), and c-Jun and c-Fos and the redox-sensitive HIF- $1 \alpha, \mathrm{Nrf} 2$, ATR/CREB are increased (499). Even although PMN are then recruited into lungs, the production of ROS by the endothelium is the initial signal.

Reperfusion further activates NADPH oxidase-2 leading to lipid peroxidation, which can be several fold more extensive than ischemia alone. Opening of an inward $\mathrm{K}^{+}$channel was accompanied with hyperpolarization and ROS as well as NO production. Mainly a PMN influx and macrophage activation contribute to that injury. There is a strong correlation between excessive oxidative stress markers and the acute donor lung injury extent, and immunological rejection including later chronic rejection in terms of bronchitis obliterans syndrome as both the major causes for lung graft failure (499) (Figure 9). 




FIGURE 9 | Pathophysiology and biological significance of pulmonary reimplantation response after lung transplantation (491-501). Adapted from Chatterjee et al (499), Whitson et al (496), Hartert et al (501), Basseri et al (498), Bharat et al (495), Bharat et al (497), Christie et al (492, 493), Huang et al (502), and King et al (491).

The success of lung transplantation is much tempered by the limited organ supply. Many potential recipients are dying on the waiting list or being removed from the list because of clinical decline (506). Groups have therefore tried to expand the donor polls using extended criteria donors, with efforts to suggest rates of PGD, bronchiolitis obliterans syndrome, early morbidity and mortality to have equivalent to those with standard criteria donors (506). Most lung grafts come from brain-dead donors, but only about $15-20 \%$ of donors provide lungs that are satisfactory for lung transplantation (506). Strategies to expand the donor pool include the use of donation after cardiocirculatory death by doing a normothermic ex vivo lung perfusion (507), resulting in a study an about $28 \%$ increase in lungs suitable for transplantation. Problems are the increased risk of perioperative hypotension, warm ischemia time, a higher rate of aspiration, and more uncertainty to predict the lung's usability for transplantation. Ex vivo assessment and reconditioning might overcome some issues in the longer term (506) (Figure 9).

As shown before, using TNF tip peptide as preventative strategy in the left-sided unilateral orthotopic rat lung transplant model of prolonged cold ischemia we could show important biological effects, as highly severe lung injury with blood gas parameters qualifying for severe ARDS could be virtually prevented by the activation of the TNF lectin-like region (8) (Figure 11). The clinical pilot study of Aigner et al. suggests relevant improvement during established PGD by the TNF tip peptide (357). Both studies underline the biological potential of the TNF lectin-like region, i.e., the cytokine's ion channel activation, thus its potent modulation of ALI, and thus its potential effect to prevent untoward long-term effects.

\section{Interstitial Lung Disease, Especially Acute Exacerbation of Idiopathic Pulmonary Fibrosis (aeIPF)}

Idiopathic pulmonary fibrosis is a chronic and progressive lung disease of unknown etiology that occurs primarily in adults in their 50s and 60s and higher. Annual incidence is about 7-16 cases per 100,000 in the USA and 0.2 - 7 per 100,000 in Europe. Prognosis is severe with a median survival of about $2-3$ years after diagnosis (508).

Acute exacerbation of idiopathic pulmonary fibrosis is a highly important disease progression of high morbidity and an extremely high mortality of 50-90\% (509). It is typically reported to have an annual incidence of 5-15 or more \%, with a higher incidence in advanced disease, and is defined as an acute worsening of dyspnea and lung function without an identifiable cause. Intriguingly, aeIPF has quite similar clinical features and similar prognosis compared with non-idiopathic causes of acute respiratory worsening in IPF such as infection or aspiration. It is, therefore, debated whether etiologies are to be separated (509). 


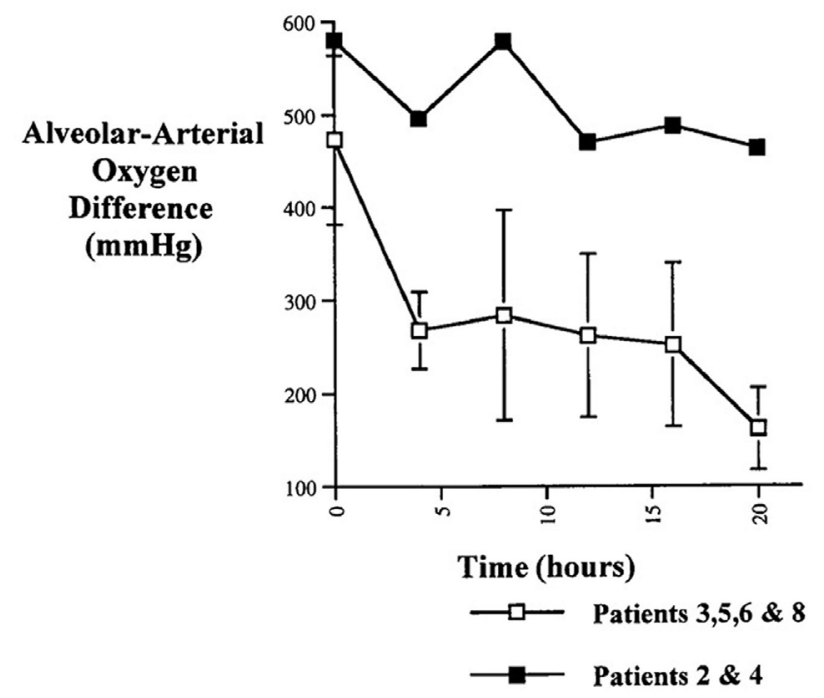

FIGURE 10 | Time course of mean AaPO2 after the onset of reperfusion pulmonary edema. Comparison of mean $\mathrm{AaPO} 2$ in four patients with intact alveolar epithelial fluid clearance (open squares) to the patients with no net alveolar epithelial fluid clearance (solid squares). The data for Patients 3 , 5,6 , and 8 are expressed as mean 6 SD. The data for Patients 2 and 4 are expressed as the average of the AaPO2 at each time point (45). Reprinted with permission of the American Thoracic Society. Copyright (c) 2017 American Thoracic Society.

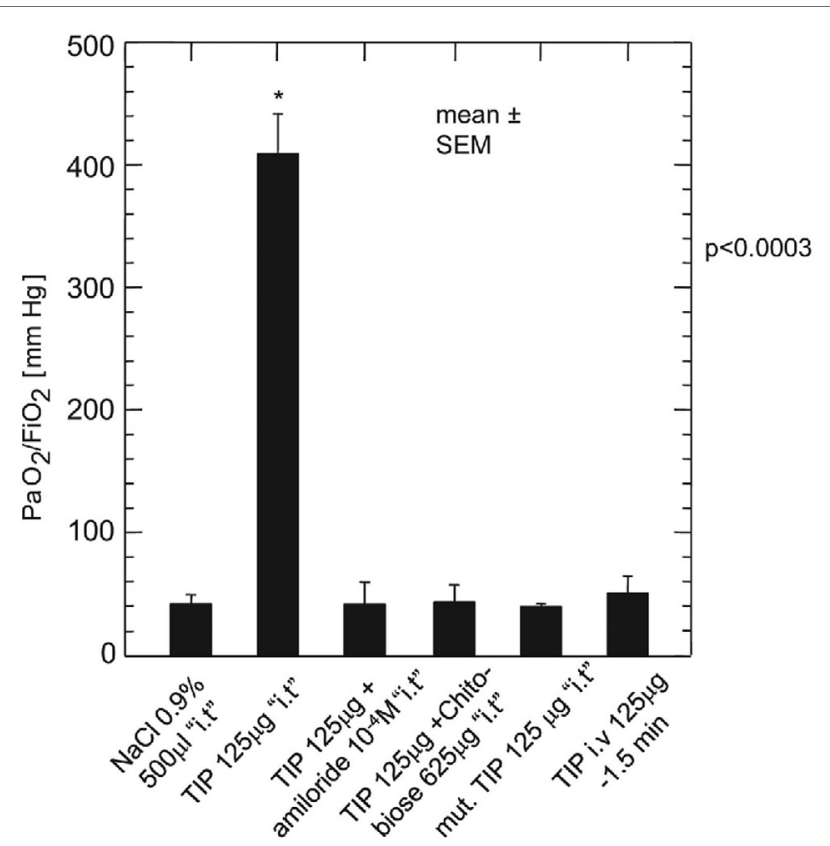

FIGURE 11 | Oxygenation at $24 \mathrm{~h}$ after transplantation. At sacrifice, $24 \mathrm{~h}$ after reperfusion of the left-sided lung transplant, the $\mathrm{PaO} 2 / \mathrm{FIO} 2$ ratio was measured after excluding the native right-sided lung by clipping the right-sided stem bronchus and right-sided pulmonary artery. The animals were tracheotomized and ventilated with an $\mathrm{FIO} 2$ of 1.0. The tumor necrosis factor tip peptide significantly increased gas exchange compared with all other study groups. ${ }^{*} p<0.003$ versus $\mathrm{NaCl}$. Data are mean $\pm \mathrm{SEM}$. i.t., intratracheally (8).
There is some similarity between aeIPF and ARDS. However, the biological backgrounds are even much less understood. Gene expression profiles mainly show primarily infections or overwhelming inflammatory etiology, but more epithelial injury and proliferation as main profile, including gene expression of CCNA2, alpha-defensin, and apoptosis. Histopathologically, diffuse alveolar damage seems frequently observed in aeIPF. This finding is similar to ARDS and also has systemic multiorgan disease consequences, as evidenced by autopsy findings (510).

A number of current pharmacotherapies are under investigation for the therapeutic challenge of aeIPF as reviewed by Juarez et al., but no substance, combination of substances, or treatment modality (such as non-invasive ventilation which seems beneficial) has demonstrated such a clear benefit to become a new standard of therapy. This leaves clinicians with polypragmatic, mainly supportive care. Novel approaches are actually developed concerning immune suppression including calcineurin inhibitors, rituximab, removal of immune cells and mediators by either therapeutic plasma exchange or haemoperfusions with polymycin-B immobilized fibers aimed to remove not primarily endotoxin, but also contributing cytokines, and maybe hemostasis modulating agents such as intravenous recombinant thrombomodulin (508). The option of modulating the inflammation and to protect barrier function with, e.g., the biological action of TNF tip region is actually conceptualized in this group of severely sick patients.

\section{Pre-eclampsia}

Pre-eclampsia refers to the new onset of the combination of hypertension and proteinuria or of hypertension and end-organ dysfunction without or with proteinuria in previously normotensive pregnant women after at least 20 weeks of gestation. About $4-5 \%$ of pregnancies worldwide are complicated with preeclampsia, and first pregnancies are more frequently associated with this disease. Together with hemorrhage, thromboembolism, and cardiovascular disease, pre-eclampsia is one of the four leading causes of maternal death, accounting for $15 \%$ of them in the Western world. Prevalence is about 1 maternal death per 100,000 live births. When pre-eclampsia occurs, the fatality rate is about 6 per 10,000. Severe acute diastolic dysfunction in severe pre-eclampsia can lead to pulmonary edema in this patient group. Maternal and fetal/placental factors seem responsible, such as abnormal trophoblast invasion of the spiral arteria of the decidua and myometrium early in pregnancy, a suboptimal uteroplacental blood flow possibly leading to high oxidative placental stress, altering placental angiogenesis, poor feto-placental vasculature and abnormal vascular reactivity. Endothelial dysfunction can be caused by systemic anti-angiogenic signals by anti-angiogenic factors. Elevated levels of soluble fms-like tyrosine kinase 1 (sFlt1; an inhibitor of vascular endothelial growth factor), reduced levels of placental growth factor (PlGF), and an increased sFlt-1: PlGF ratio have been reported both in women with established pre-eclampsia and in women before the development of preeclampsia (511). This is moreover accompanied by increased proinflammatory cytokine production, which in turn promotes renal and pulmonary barrier dysfunction and impaired ion channel activity. As a consequence, pulmonary edema is a severe feature 
of the disease. In this case, the edema can be multifactorial, due to left heart failure, and thus excessive pulmonary vascular hydrostatic pressure, to decreased plasma oncotic pressure, to capillary leak, or to iatrogenic volume overload $(511,512)$.

\section{High-Altitude Pulmonary Edema}

About 100 million people live at altitudes greater than $2,500 \mathrm{~m}$, about 15 million above 3,000 $\mathrm{m}$, and some above 5,000 $\mathrm{m}$ (384). Most have developed the ability to live and reproduce at elevation as high as 5,000 $\mathrm{m}$, but in some cases, develop chronic medical problems due to their high-altitude residence. At 5,500 $\mathrm{m}$ the pressure is about only half the normal. Furthermore, many lowlanders venture to high altitude for work and recreation. These more acute exposures also pose the hazards of acute altitude illness, e.g., in Colorado skiers in $15-40 \%$ of them with an incidence of HAPE then of $0.1-1 \%$. The prevalence of HAPE depends on an individual's susceptibility, the rate of ascent, the final altitude, but also heavy and prolonged exercise, and is higher in male. At altitudes of 4,500 $\mathrm{m}$ the prevalence is between 0.2 and $6 \%$, and at 5,500 $\mathrm{m}$ between 2 and 15\% (385). Many adaptive processes can vastly reduce the risk of such sickness. Susceptibility to altitude illness varies considerably between individuals, but for a single individual, the symptoms are often reproducible given the same rate of ascent. High-altitude pulmonary odemea is the most important complication of high-altitude illness and its most common cause of death. It typically manifests with 2-4 days of ascent to altitudes above $2,400 \mathrm{~m}$, most commonly beginning on the second night. In the early stage of disease, decreased exercise performance occurs and individuals require increased amount of time to recover from exertions. Individuals also complained of fatigue, weakness, and persistent dry cough, possibly combined with symptoms of acute sickness. As the disease progresses, individuals become short of breath with minimal exertion. Dyspnea at rest, audible chest congestion, generalized pallor, nail bed cyanosis and production of pink frothy sputum are late findings in severe disease. Even in the absence of concurrent high-altitude cerebral edema, severe hypoxemia may produce mental changes, ataxia, and altered levels of consciousness. In general blood gas analysis reveals severe hypoxemia. Pulmonary arterial pressure is high, but pulmonary wedge pressure is normal, and heart size is not increased. Although the mechanism underlying HAPE remains incompletely understood, it appears that the elevated pulmonary artery pressure plays a pivotal role in the process. Multiple studies demonstrated that susceptible individuals have abnormally high pulmonary artery pressure in response to hypoxic breathing, during normoxic and hypoxic exercise, and on high altitude before the onset of edema. Increased sympathetic tone, and alteration in vasoactive mediators-like endothelin-1, NO produced by pulmonary ECs may also lead to stronger hypoxic pulmonary vasoconstriction (384). In autopsies, a red cell rich proteinaceous alveolar exudate with hyaline membrane is characteristic. In all autopsies, areas of pneumonitis with neutrophil accumulation but no evidence of bacterial accumulation has been observed. Most reports mentioned capillary and arterial thrombi, fibrin deposits, hemorrhage, and infarcts. Uneven hypoxic vasoconstriction is discussed. Uneven perfusion is suggested clinically by the typical patchy radiographic appearance and by MRI studies in patients together with hypoxic blood gas parameters which demonstrates greater heterogeneous regional perfusion in HAPE-susceptible subjects (384). The estimated death rate of altitude illness is about $7.7 / 100,000$ trekkers, with increasing mortality during the last decade (386).

Treatment of HAPE consists, if ever possible, in descent from altitude, rest, oxygen supplementation, and administration of drugs such as corticosteroids and furosemide.

Prophylactic inhalation the $\beta 2 \mathrm{AR}$ agonist salmeterol decreased the HAPE incidence by more than $50 \%$ (387). The most pertinent explanation was that salmeterol would enhance the clearance of alveolar fluid since $\beta$-adrenergic agonists upregulate the clearance of alveolar fluid by stimulating transepithelial sodium transport. This hypothesis is supported by the fact that the level of sodium transport in the respiratory epithelium is lower in patients susceptible to HAPE. However, the study results cannot exclude the possibility that the beta 2 agonist could have modulated vascular permeability or the hemodynamic response associated with hypoxemia and HAPE (4).

In an experimental rat model simulating HAPE by hypobaric and hypoxic conditions equivalent to an altitude of $4,500 \mathrm{~m}$ with exhaustive treadmill exercise of $15 \mathrm{~m}$ per minute for $24 \mathrm{~h}$, then for an equivalent of altitude of 6,000 $\mathrm{m}$ for further $48 \mathrm{~h}$, it has been shown that the TNF tip peptide reduced pulmonary edema and increased the TJ occluding expression compared to high-altitude controls, dexamethasone, and aminophylline treated control animals (7). Compared to untreated high-altitude control animals, TNF tip peptide significantly lowered levels of the inflammatory cytokines TNF, IL-1 $\beta$, IL-6 and IL-8 in bronchoalveolar lavage. TNF tip peptide-treated animals experienced less pulmonary edema also compared to dexamethasone-treated animals, and was more effective than its comparators in reduction of bronchoalveolar lavage protein content and inflammatory parameters (7). The higher expression of occludin may have translated in an increased stability of the alveolar-capillary barrier, probably related to the reduction in the extent of protein leakage in TNF tip peptide-treated animals. The results suggest that the biologic potential of the TNF tip region is more active in this model than dexamethasone as standard therapy on one hand, and as the glucocorticosteroids (7). The model suggests that HAPE can be treated with TNF tip peptide at least in a part of patients affected, and clinical studies are underway.

However, inhaled budesonide seems not consistently able to prevent acute mountain sickness and HAPE (513).

\section{SUMMARY AND CONCLUSION}

Alveolar fluid reabsorption is of high clinical importance in both cardiac and non-cardiac edema. Clinically, a conservative fluid strategy in ARDS patients resulted in more ventilator-free days (514). There is evidence that lower vascular pressures reduce pro-inflammatory pathways (515), and in chronic hydrostatic pulmonary edema tissue remodeling ensues (516).

Recent studies of cytokine-ion channel interactions have clearly shown that the concept of ion channel modulation to improve AFC has to be broadened, also taking into account 
previously ignored functions of these mediators. The concept of active interactions between barrier function and ion transporters to maintain lung fluid balance plays a pivotal biological role. TNF's lectin-like domain, mimicked by the TNF tip peptide, was demonstrated to strengthen capillary barrier function in the presence of bacterial toxins in vitro and in vivo. Indeed, influx, efflux, and tightness of the EC layer are all biologically interrelated. Such a relationship is also present in the alveolar epithelium with interactions with ion transporters and TJs (11). These observations suggest that the biologic potential of ion channel modulation with drugs or peptides is more relevant than initially presumed.

A conceptual problem in ALI and other inflammatory conditions is how fluid reabsorption can function in such an "un-tight system" as in partially destroyed endothelial-interstitial or interstitial-alveolar barriers, and what is the expression level of ion channels in those conditions (25). The same may hold true in the context of hypoxia and the decreased expression of $\mathrm{ENaC}$. Regeneration and repair of injured, apoptotic or necrotic endothelial or AECs can be fostered endogenously by local or bone-marrow derived precursors or by exogenously administered factors, as formerly studied in animal models using progenitor cell populations and stimulants. Clinical refinements are underway and update outcome parameters, such as AFC (517).

In clinical situations with cardiogenic as well as with noncardiogenic pulmonary edema, i.e., ALI and ARDS, we have to be extremely cautious with prescribing drugs that might interfere with alveolar fluid transports or inflammation. Furosemide might further be the mainstay of diuretic drug and the alveolar flooding stopper especially in cardiogenic edema due to its effect on NKCC1 and CFTR. Amiloride should not be taken. Many clinical questions will be open around beta blocking agents as well as beta stimulating agents in the context of pulmonary edema and will probably depend on their indication. cAMP may play some role, but from which point those two drug classes are counterproductive, remains actually open.

There has been much work focused on one ion channel without considering the interconnection between major biological

\section{REFERENCES}

1. Staub NC. Pulmonary edema. Physiol Rev (1974) 54(3):678-811.

2. Ware LB, Matthay MA. Clinical practice. Acute pulmonary edema. N Engl J Med (2005) 353(26):2788-96. doi:10.1056/NEJMcp052699

3. Weibel ER, Taylor CR. Design and structure of the human lung. In: Fishman A, editor. Pulmonary Diseases and Disorders. New York: McGraw-Hill (1988). p. 11-60.

4. Berthiaume Y, Matthay MA. Alveolar edema fluid clearance and acute lung injury. Respir Physiol Neurobiol (2007) 159(3):350-9. doi:10.1016/ j.resp.2007.05.010

5. Weidenfeld S, Kuebler WM. Cytokine-regulation of Na+-K+-Cl- cotransporter 1 and cystic fibrosis transmembrane conductance regulator-potential role in pulmonary inflammation and edema formation. Front Immunol (2017) 8:393. doi:10.3389/fimmu.2017.00393

6. Hoffman O, Burns N, Vadasz I, Eltzschig HK, Edwards MG, Vohwinkel CU. Detrimental ELAVL-1/HuR-dependent GSK3beta mRNA stabilization impairs resolution in acute respiratory distress syndrome. PLoS One (2017) 12(2):e0172116. doi:10.1371/journal.pone.0172116

7. Zhou Q, Wang D, Liu Y, Yang X, Lucas R, Fischer B. Solnatide demonstrates profound therapeutic activity in a rat model of pulmonary edema induced ion channels or its modulators, which may limit the validity of conclusions or findings of much published work. In future research it would be important to try to better integrate these channels, as well as their interactions with cytokines present in the lung milieu during the various pathologies. Many parallels exist between different organ systems and ion channels, underlining that interdisciplinary network is promising.

As shown in lung transplant primary graft failure, and thus probably also true in ARDS, ALI causes important and systemic long-term injury, especially brain injury. The critical step of high ethical impact for the scientific community is to expand integrative translational research in terms of clinical investigation with the known targets to improve clinical outcome. This is especially important in lung transplantation, as donor shortage still leaves many patients worldwide dying without this therapeutic option, and possibly in ALI and ARDS.

\section{AUTHOR CONTRIBUTIONS}

All authors significantly contributed to the conceptual work, the writing and editing of the work.

\section{ACKNOWLEDGMENTS}

The authors acknowledge Sarah Lea Hipp, Graphics and Typography, www.sarahleahipp.ch, for designing and providing the Figures 2, 4-8. The authors also acknowledge the financial support of the Lungen-und Atmungsstiftung, Bern. The work is dedicated to Ellen Hamacher who died during the finalization of the manuscript.

\section{FUNDING}

This work was supported by the Lungen- und Atmungsstiftung Bern. This work was further supported by Extramural Success Award from the Vice President for Research at Augusta University (to RL), by ADA grant \#1 -16-IBS-196 (to RL). RL is a Mercator Fellow of the German Research Foundation (DFG).

by acute hypobaric hypoxia and exercise. Chest (2017) 151(3):658-67. doi:10.1016/j.chest.2016.10.030

8. Hamacher J, Stammberger U, Roux J, Kumar S, Yang G, Xiong C, et al. The lectin-like domain of tumor necrosis factor improves lung function after rat lung transplantation - potential role for a reduction in reactive oxygen species generation. Crit Care Med (2010) 38(3):871-8. doi:10.1097/CCM. 0b013e3181cdf725

9. Czikora I, Alli AA, Sridhar S, Matthay MA, Pillich H, Hudel M, et al. Epithelial sodium channel- $\alpha$ mediates the protective effect of the TNF-derived TIP peptide in pneumolysin-induced endothelial barrier dysfunction. Front Immunol (2017) 8:842. doi:10.3389/fimmu.2017.00842

10. Lucas R, Yang G, Gorshkov BA, Zemskov EA, Sridhar S, Umapathy NS, et al. Protein kinase C-alpha and arginase I mediate pneumolysin-induced pulmonary endothelial hyperpermeability. Am J Respir Cell Mol Biol (2012) 47(4): 445-53. doi:10.1165/rcmb.2011-0332OC

11. Brune K, Frank J, Schwingshackl A, Finigan J, Sidhaye VK. Pulmonary epithelial barrier function: some new players and mechanisms. Am J Physiol Lung Cell Mol Physiol (2015) 308(8):L731-45. doi:10.1152/ajplung.00309.2014

12. Hummler E, Barker P, Gatzy J, Beermann F, Verdumo C, Schmidt A, et al. Early death due to defective neonatal lung liquid clearance in alpha-ENaCdeficient mice. Nat Genet (1996) 12(3):325-8. doi:10.1038/ng0396-325 
13. Huppmann S, Lankes E, Schnabel D, Buhrer C. Unimpaired postnatal respiratory adaptation in a preterm human infant with a homozygous $\mathrm{ENaC}-$ alpha unit loss-of-function mutation. JPerinatol (2011) 31(12):802-3. doi:10.1038/jp.2011.46

14. O'Brodovich H. Pulmonary edema in infants and children. Curr Opin Pediatr (2005) 17(3):381-4. doi:10.1097/01.mop.0000159780.42572.6c

15. Verghese GM, Ware LB, Matthay BA, Matthay MA. Alveolar epithelial fluid transport and the resolution of clinically severe hydrostatic pulmonary edema. J Appl Physiol (1985) (1999) 87(4):1301-12.

16. Solymosi EA, Kaestle-Gembardt SM, Vadasz I, Wang L, Neye N, Chupin CJ, et al. Chloride transport-driven alveolar fluid secretion is a major contributor to cardiogenic lung edema. Proc Natl Acad Sci U S A (2013) 110(25): E2308-16. doi:10.1073/pnas.1216382110

17. Matthay MA, Clerici C. Alveolar epithelial and fluid transport. In: Broaddus VC, Mason RJ, Ernst JD, King TE Jr., Lazarus SC, Murray JF, Nadel JA, Slutsky SA, Gotway MB, editors. Murray \& Nadel's Textbook of Respiratory Medicine. Philadelphia: Elsevier Saunders (2016). p. 150-6.

18. Ribeiro CM, Marchiori E, Rodrigues R, Gasparetto E, Souza AS Jr., Escuissato D, et al. Hydrostatic pulmonary edema: high-resolution computed tomography aspects. J Bras Pneumol (2006) 32(6):515-22. doi:10.1590/ S1806-37132006000600008

19. Platz E, Jhund PS, Campbell RT, McMurray JJ. Assessment and prevalence of pulmonary oedema in contemporary acute heart failure trials: a systematic review. Eur J Heart Fail (2015) 17(9):906-16. doi:10.1002/ejhf.321

20. Roger VL. Epidemiology of heart failure. Circ Res (2013) 113(6):646-59. doi:10.1161/circresaha.113.300268

21. Azzam ZS, Kinaneh S, Bahouth F, Ismael-Badarneh R, Khoury E, Abassi Z. Involvement of cytokines in the pathogenesis of salt and water imbalance in congestive heart failure. Front Immunol (2017) 8:716. doi:10.3389/fimmu. 2017.00716

22. Gray A, Goodacre S, Newby DE, Masson M, Sampson F, Nicholl J, et al. Noninvasive ventilation in acute cardiogenic pulmonary edema. $N$ Engl J Med (2008) 359(2):142-51. doi:10.1056/NEJMoa0707992

23. Bove PF, Grubb BR, Okada SF, Ribeiro CM, Rogers TD, Randell SH, et al. Human alveolar type II cells secrete and absorb liquid in response to local nucleotide signaling. J Biol Chem (2010) 285(45):34939-49. doi:10.1074/jbc. M110.162933

24. Schmieder RE, Messerli FH, deCarvalho JG, Husserl FE. Immediate hemodynamic response to furosemide in patients undergoing chronic hemodialysis. Am J Kidney Dis (1987) 9(1):55-9. doi:10.1016/S0272-6386(87)80162-2

25. Matthay MA. Resolution of pulmonary edema. Thirty years of progress. Am J Respir Crit Care Med (2014) 189(11):1301-8. doi:10.1164/rccm.201403$0535 \mathrm{OE}$

26. Lourenco P, Paulo Araujo J, Paulo C, Mascarenhas J, Frioes F, Azevedo A, et al. Higher C-reactive protein predicts worse prognosis in acute heart failure only in noninfected patients. Clin Cardiol (2010) 33(11):708-14. doi:10.1002/ clc. 20812

27. Mann DL, McMurray JJ, Packer M, Swedberg K, Borer JS, Colucci WS, et al. Targeted anticytokine therapy in patients with chronic heart failure: results of the Randomized Etanercept Worldwide Evaluation (RENEWAL). Circulation (2004) 109(13):1594-602. doi:10.1161/01.CIR.0000124490.27666.B2

28. Chung ES, Packer M, Lo KH, Fasanmade AA, Willerson JT, Anti-TNF Therapy Against Congestive Heart Failure Investigators. Randomized, doubleblind, placebo-controlled, pilot trial of infliximab, a chimeric monoclonal antibody to tumor necrosis factor-alpha, in patients with moderate-to-severe heart failure: results of the anti-TNF Therapy Against Congestive Heart Failure (ATTACH) trial. Circulation (2003) 107(25):3133-40. doi:10.1161/ 01.CIR.0000077913.60364.D2

29. Cacciapaglia F, Salvatorelli E, Minotti G, Afeltra A, Menna P. Low level tumor necrosis factor-alpha protects cardiomyocytes against high level tumor necrosis factor-alpha: brief insight into a beneficial paradox. Cardiovasc Toxicol (2014) 14(4):387-92. doi:10.1007/s12012-014-9257-z

30. De Vito P. The sodium/hydrogen exchanger: a possible mediator of immunity. Cell Immunol (2006) 240(2):69-85. doi:10.1016/j.cellimm.2006.07.001

31. Thompson BT, Chambers RC, Liu KD. Acute respiratory distress syndrome. N Engl J Med (2017) 377(6):562-72. doi:10.1056/NEJMra1608077

32. Pittet JF, Mackersie RC, Martin TR, Matthay MA. Biological markers of acute lung injury: prognostic and pathogenetic significance. Am J Respir Crit Care Med (1997) 155(4):1187-205. doi:10.1164/ajrccm.155.4.9105054
33. Ware LB, Matthay MA. Alveolar fluid clearance is impaired in the majority of patients with acute lung injury and the acute respiratory distress syndrome. Am J Respir Crit Care Med (2001) 163(6):1376-83. doi:10.1164/ ajrccm.163.6.2004035

34. Millar FR, Summers C, Griffiths MJ, Toshner MR, Proudfoot AG. The pulmonary endothelium in acute respiratory distress syndrome: insights and therapeutic opportunities. Thorax (2016) 71(5):462-73. doi:10.1136/thoraxjnl2015-207461

35. Khan SU, Salloum J, O’Donovan PB, Mascha EJ, Mehta AC, Matthay MA, et al. Acute pulmonary edema after lung transplantation: the pulmonary reimplantation response. Chest (1999) 116(1):187-94. doi:10.1378/chest. 116.1.187

36. Frank JA, Matthay MA. TGF-beta and lung fluid balance in ARDS. Proc Natl Acad Sci U S A (2014) 111(3):885-6. doi:10.1073/pnas.1322478111

37. Braun C, Hamacher J, Morel DR, Wendel A, Lucas R. Dichotomal role of TNF in experimental pulmonary edema reabsorption. JImmunol (2005) 175(5):3402-8. doi:10.4049/jimmunol.175.5.3402

38. Yang G, Hamacher J, Gorshkov B, White R, Sridhar S, Verin A, et al. The dual role of TNF in pulmonary edema. J Cardiovasc Dis Res (2010) 1(1):29-36. doi:10.4103/0975-3583.59983

39. Hamacher J, Lucas R, Lijnen HR, Buschke S, Dunant Y, Wendel A, et al. Tumor necrosis factor-alpha and angiostatin are mediators of endothelial cytotoxicity in bronchoalveolar lavages of patients with acute respiratory distress syndrome. Am J Respir Crit Care Med (2002) 166(5):651-6. doi:10.1164/rccm.2109004

40. Matute-Bello G, Liles WC, Frevert CW, Dhanireddy S, Ballman K, Wong V, et al. Blockade of the Fas/FasL system improves pneumococcal clearance from the lungs without preventing dissemination of bacteria to the spleen. J Infect Dis (2005) 191(4):596-606. doi:10.1086/427261

41. Chen W, Janz DR, Bastarache JA, May AK, O’Neal HR Jr., Bernard GR, et al. Prehospital aspirin use is associated with reduced risk of acute respiratory distress syndrome in critically ill patients: a propensity-adjusted analysis. Crit Care Med (2015) 43(4):801-7. doi:10.1097/CCM.0000000000000789

42. Sznajder JI. Alveolar edema must be cleared for the acute respiratory distress syndrome patient to survive. Am J Respir Crit Care Med (2001) 163(6): 1293-4. doi:10.1164/ajrccm.163.6.ed1801d

43. Matthay MA, Zemans RL. The acute respiratory distress syndrome: pathogenesis and treatment. Annu Rev Pathol (2011) 6:147-63. doi:10.1146/ annurev-pathol-011110-130158

44. Zeyed YF, Bastarache JA, Matthay MA, Ware LB. The severity of shock is associated with impaired rates of net alveolar fluid clearance in clinical acute lung injury. Am J Physiol Lung Cell Mol Physiol (2012) 303(6):L550-5. doi:10.1152/ajplung.00190.2012

45. Ware LB, Golden JA, Finkbeiner WE, Matthay MA. Alveolar epithelial fluid transport capacity in reperfusion lung injury after lung transplantation. Am J Respir Crit Care Med (1999) 159(3):980-8. doi:10.1164/ajrccm.159.3.9802105

46. Matthay MA, Folkesson HG, Clerici C. Lung epithelial fluid transport and the resolution of pulmonary edema. Physiol Rev (2002) 82(3):569-600. doi:10.1152/physrev.00003.2002

47. Johnson MD, Widdicombe JH, Allen L, Barbry P, Dobbs LG. Alveolar epithelial type I cells contain transport proteins and transport sodium, supporting an active role for type I cells in regulation of lung liquid homeostasis. Proc Natl Acad Sci U S A (2002) 99(4):1966-71. doi:10.1073/pnas.042689399

48. Ma T, Fukuda N, Song Y, Matthay MA, Verkman AS. Lung fluid transport in aquaporin-5 knockout mice. J Clin Invest (2000) 105(1):93-100. doi:10.1172/ JCI8258

49. Clements JA. Lung surfactant: a personal perspective. Annu Rev Physiol (1997) 59:1-21. doi:10.1146/annurev.physiol.59.1.1

50. Berthiaume Y, Lesur O, Dagenais A. Treatment of adult respiratory distress syndrome: plea for rescue therapy of the alveolar epithelium. Thorax (1999) 54(2):150-60. doi:10.1136/thx.54.2.150

51. Matalon ST, Shoenfeld Y, Blank M, Yacobi S, Blumenfeld Z, Ornoy A. The effects of IgG purified from women with SLE and associated pregnancy loss on rat embryos in culture. Am J Reprod Immunol (2002) 48(5):296-304. doi:10.1034/j.1600-0897.2002.01084.x

52. Althaus M, Clauss WG, Fronius M. Amiloride-sensitive sodium channels and pulmonary edema. Pulm Med (2011) 2011:830320. doi:10.1155/2011/830320

53. Althaus M, Pichl A, Clauss WG, Seeger W, Fronius M, Morty RE. Nitric oxide inhibits highly selective sodium channels and the $\mathrm{Na}+\mathrm{K}+$-ATPase in $\mathrm{H} 441$ cells. Am J Respir Cell Mol Biol (2011) 44(1):53-65. doi:10.1165/2009-0335oc 
54. Czikora I, Alli A, Bao HF, Kaftan D, Sridhar S, Apell HJ, et al. A novel tumor necrosis factor-mediated mechanism of direct epithelial sodium channel activation. Am J Respir Crit Care Med (2014) 190(5):522-32. doi:10.1164/ rccm.201405-0833OC

55. Gon Y, Wood MR, Kiosses WB, Jo E, Sanna MG, Chun J, et al. S1P3 receptor-induced reorganization of epithelial tight junctions compromises lung barrier integrity and is potentiated by TNF. Proc Natl Acad Sci U S A (2005) 102(26):9270-5. doi:10.1073/pnas.0501997102

56. Bachofen M, Weibel ER. Alterations of the gas exchange apparatus in adult respiratory insufficiency associated with septicemia 1, 2. Am J Respir Crit Care Med (1977) 116(4):589-615. doi:10.1164/arrd.1977.116.4.589

57. Matute-Bello G, Liles WC, Steinberg KP, Kiener PA, Mongovin S, Chi EY, et al. Soluble Fas ligand induces epithelial cell apoptosis in humans with acute lung injury (ARDS). J Immunol (1999) 163(4):2217-25.

58. Martin TR, Hagimoto N, Nakamura M, Matute-Bello G. Apoptosis and epithelial injury in the lungs. Proc Am Thorac Soc (2005) 2(3):214-20. doi:10.1513/pats.200504-031AC

59. Hamilton BC, Kukreja J, Ware LB, Matthay MA. Protein biomarkers associated with primary graft dysfunction following lung transplantation. Am J Physiol Lung Cell Mol Physiol (2017) 312(4):L531-41. doi:10.1152/ajplung. 00454.2016

60. Sharp C, Millar AB, Medford AR. Advances in understanding of the pathogenesis of acute respiratory distress syndrome. Respiration (2015) 89(5):420-34. doi:10.1159/000381102

61. Patterson CE, Lum H. Update on pulmonary edema: the role and regulation of endothelial barrier function. Endothelium (2001) 8(2):75-105. doi:10.3109/10623320109165319

62. Trzeciak S, Dellinger RP, Parrillo JE, Guglielmi M, Bajaj J, Abate NL, et al. Early microcirculatory perfusion derangements in patients with severe sepsis and septic shock: relationship to hemodynamics, oxygen transport, and survival. Ann Emerg Med (2007) 49(1):e1-2. doi:10.1016/j. annemergmed.2006.08.021

63. Nuckton TJ, Alonso JA, Kallet RH, Daniel BM, Pittet JF, Eisner MD, et al. Pulmonary dead-space fraction as a risk factor for death in the acute respiratory distress syndrome. N Engl J Med (2002) 346(17):1281-6. doi:10.1056/ NEJMoa012835

64. Gill SE, Rohan M, Mehta S. Role of pulmonary microvascular endothelial cell apoptosis in murine sepsis-induced lung injury in vivo. Respir Res (2015) 16:109. doi:10.1186/s12931-015-0266-7

65. Mutunga M, Fulton B, Bullock R, Batchelor A, Gascoigne A, Gillespie JI, et al. Circulating endothelial cells in patients with septic shock. Am J Respir Crit Care Med (2001) 163(1):195-200. doi:10.1164/ajrccm.163.1.9912036

66. Dudek SM, Garcia JG. Cytoskeletal regulation of pulmonary vascular permeability. J Appl Physiol (1985) (2001) 91(4):1487-500.

67. Petrache I, Birukova A, Ramirez SI, Garcia JG, Verin AD. The role of the microtubules in tumor necrosis factor-alpha-induced endothelial cell permeability. Am J Respir Cell Mol Biol (2003) 28(5):574-81. doi:10.1165/ rcmb.2002-0075OC

68. Lucas R, Verin AD, Black SM, Catravas JD. Regulators of endothelial and epithelial barrier integrity and function in acute lung injury. Biochem Pharmacol (2009) 77(12):1763-72. doi:10.1016/j.bcp.2009.01.014

69. Delany NS, Hurle M, Facer P, Alnadaf T, Plumpton C, Kinghorn I, et al. Identification and characterization of a novel human vanilloid receptor-like protein, VRL-2. Physiol Genomics (2001) 4(3):165-74.

70. Stevens T. Functional and molecular heterogeneity of pulmonary endothelial cells. Proc Am Thorac Soc (2011) 8(6):453-7. doi:10.1513/ pats.201101-004MW

71. Herold S, Gabrielli NM, Vadasz I. Novel concepts of acute lung injury and alveolar-capillary barrier dysfunction. Am J Physiol Lung Cell Mol Physiol (2013) 305(10):L665-81. doi:10.1152/ajplung.00232.2013

72. Alberts B, Johnson A, Lewis J, Morgan D, Raff M, Roberts K, et al. Molecular Biology of the Cell. New York: Garland Science. Taylor \& Francis Group (2014).

73. Sznajder JI, Factor P, Ingbar DH. Invited review: lung edema clearance: role of $\mathrm{Na}(+)-\mathrm{K}(+)$-ATPase. J Appl Physiol (1985) (2002) 93(5):1860-6. doi:10.1152/japplphysiol.00022.2002

74. Purves D, Augustine G, Fitzpatrick D. Neuroscience. In: Purves D, Augustine G, Fitzpatrick D, Katz L, LaMatina A, McNamara J, Williams S, editors. Active
Transporters Create and Maintain Ion Gradients. 2nd ed. Sunderland: Sinauer Associates (2001). p. 86-7.

75. Vivona ML, Matthay M, Chabaud MB, Friedlander G, Clerici C. Hypoxia reduces alveolar epithelial sodium and fluid transport in rats: reversal by beta-adrenergic agonist treatment. Am J Respir Cell Mol Biol (2001) 25(5):554-61. doi:10.1165/ajrcmb.25.5.4420

76. Urner M, Herrmann IK, Booy C, Roth-Z' Graggen B, Maggiorini M, Beck-Schimmer B. Effect of hypoxia and dexamethasone on inflammation and ion transporter function in pulmonary cells. Clin Exp Immunol (2012) 169(2):119-28. doi:10.1111/j.1365-2249.2012.04595.x

77. Gille T, Randrianarison-Pellan N, Goolaerts A, Dard N, Uzunhan Y, Ferrary E, et al. Hypoxia-induced inhibition of epithelial $\mathrm{Na}(+)$ channels in the lung. Role of Nedd4-2 and the ubiquitin-proteasome pathway. Am J Respir Cell Mol Biol (2014) 50(3):526-37. doi:10.1165/rcmb.2012$0518 \mathrm{OC}$

78. Vadasz I, Raviv S, Sznajder JI. Alveolar epithelium and Na,K-ATPase in acute lung injury. Intensive Care Med (2007) 33(7):1243-51. doi:10.1007/ s00134-007-0661-8

79. Gwozdzinska P, Buchbinder BA, Mayer K, Herold S, Morty RE, Seeger W, et al. Hypercapnia impairs ENaC Cell surface stability by promoting phosphorylation, polyubiquitination and endocytosis of beta-ENaC in a human alveolar epithelial cell line. Front Immunol (2017) 8:591. doi:10.3389/ fimmu.2017.00591

80. Vadasz I, Sznajder JI. Gas exchange disturbances regulate alveolar fluid clearance during acute lung injury. Front Immunol (2017) 8:757. doi:10.3389/ fimmu.2017.00757

81. Kohnlein T, Windisch W, Kohler D, Drabik A, Geiseler J, Hartl S, et al. Non-invasive positive pressure ventilation for the treatment of severe stable chronic obstructive pulmonary disease: a prospective, multicentre, randomised, controlled clinical trial. Lancet Respir Med (2014) 2(9):698-705. doi:10.1016/S2213-2600(14)70153-5

82. Windisch W, Storre JH, Kohnlein T. Nocturnal non-invasive positive pressure ventilation for COPD. Expert Rev Respir Med (2015) 9(3):295-308. doi:10.1586/17476348.2015.1035260

83. Schwarz SB, Magnet FS, Windisch W. Why high-intensity nppv is favourable to low-intensity NPPV: clinical and physiological reasons. COPD (2017) 14(4):389-95. doi:10.1080/15412555.2017.1318843

84. Jentsch TJ, Hubner CA, Fuhrmann JC. Ion channels: function unravelled by dysfunction. Nat Cell Biol (2004) 6(11):1039-47. doi:10.1038/ncb11041039

85. Hübner CA, Jentsch TJ. Ion channel diseases. Hum Mol Genet (2002) 11(20): 2435-45. doi:10.1093/hmg/11.20.2435

86. Eaton DC, Helms MN, Koval M, Bao HF, Jain L. The contribution of epithelial sodium channels to alveolar function in health and disease. Annu Rev Physiol (2009) 71:403-23. doi:10.1146/annurev.physiol.010908.163250

87. Canessa CM, Horisberger JD, Rossier BC. Epithelial sodium channel related to proteins involved in neurodegeneration. Nature (1993) 361(6411):467-70. doi: $10.1038 / 361467 \mathrm{a} 0$

88. Kellenberger S, Schild L. International union of basic and clinical pharmacology. XCI. structure, function, and pharmacology of acid-sensing ion channels and the epithelial Na+ channel. Pharmacol Rev (2015) 67(1):1-35. doi:10.1124/pr.114.009225

89. Canessa CM, Schild L, Buell G, Thorens B, Gautschi I, Horisberger JD, et al. Amiloride-sensitive epithelial $\mathrm{Na}+$ channel is made of three homologous subunits. Nature (1994) 367(6462):463-7. doi:10.1038/367463a0

90. Waldmann R, Champigny G, Bassilana F, Voilley N, Lazdunski M. Molecular cloning and functional expression of a novel amiloride-sensitive $\mathrm{Na}+$ channel. J Biol Chem (1995) 270(46):27411-4. doi:10.1074/jbc.270.46.27411

91. Ji HL, Su XF, Kedar S, Li J, Barbry P, Smith PR, et al. Delta-subunit confers novel biophysical features to alpha beta gamma-human epithelial sodium channel (ENaC) via a physical interaction. JBiol Chem (2006) 281(12): 8233-41. doi:10.1074/jbc.M512293200

92. Matalon S, Lazrak A, Jain L, Eaton DC. Invited review: biophysical properties of sodium channels in lung alveolar epithelial cells. J Appl Physiol (1985) (2002) 93(5):1852-9. doi:10.1152/japplphysiol.01241.2001

93. McNicholas CM, Canessa CM. Diversity of channels generated by different combinations of epithelial sodium channel subunits. J Gen Physiol (1997) 109(6):681-92. doi:10.1085/jgp.109.6.681 
94. Chalfant M, Denton J, Langloh A, Karlson K, Loffing J, Benos D, et al. The $\mathrm{NH} 2$ terminus of the epithelial sodium channel contains an endocytic motif. J Biol Chem (1999) 274:32889-96. doi:10.1074/jbc.274.46.32889

95. Ji H-L, Zhao R-Z, Chen Z-X, Shetty S, Idell S, Matalon S. $\delta$ ENaC: a novel divergent amiloride-inhibitable sodium channel. Am J Physiol Lung Cell Mol Physiol (2012) 303(12):L1013-26. doi:10.1152/ajplung.00206.2012

96. GuidotDM, Folkesson HG, Jain L, SznajderJI, PittetJ-F, Matthay MA. Integrating acute lung injury and regulation of alveolar fluid clearance. Am J Physiol Lung Cell Mol Physiol (2006) 291(3):L301. doi:10.1152/ajplung.00153.2006

97. Czikora I, Sridhar S, Alli A, Verin A, Chakraborty T, Fulton D, et al. ENaC-a mediates the protective effect of the TNF-derived TIP peptide in pneumolysininduced capillary barrier dysfunction. FASEB J (2017) 31(1 Suppl):978.6.

98. Garty H, Palmer LG. Epithelial sodium channels: function, structure, and regulation. Physiol Rev (1997) 77(2):359-96.

99. Elia N, Tapponnier M, Matthay MA, Hamacher J, Pache JC, Brundler MA, et al. Functional identification of the alveolar edema reabsorption activity of murine tumor necrosis factor-alpha. Am J Respir Crit Care Med (2003) 168(9):1043-50. doi:10.1164/rccm.200206-618OC

100. Hazemi P, Tzotzos SJ, Fischer B, Andavan GSB, Fischer H, Pietschmann H, et al. Essential structural features of TNF- $\alpha$ lectin-like domain derived peptides for activation of amiloride-sensitive sodium current in A549 cells. J Med Chem (2010) 53(22):8021-9. doi:10.1021/jm100767p

101. Sugita M, Ferraro P, Dagenais A, Clermont ME, Barbry P, Michel RP, et al. Alveolar liquid clearance and sodium channel expression are decreased in transplanted canine lungs. Am J Respir Crit Care Med (2003) 167(10): 1440-50. doi:10.1164/rccm.200204-312OC

102. Lucas R, Yue Q, Alli A, Duke BJ, Al-Khalili O, Thai TL, et al. The lectinlike domain of TNF increases $\mathrm{ENaC}$ open probability through a novel site at the interface between the second transmembrane and C-terminal domains of the alpha-subunit. J Biol Chem (2016) 291(45):23440-51. doi:10.1074/ jbc.M116.718163

103. Trac PT, Thai TL, Linck V, Zou L, Greenlee M, Yue Q, et al. Alveolar nonselective channels are ASIC1a/alpha-ENaC channels and contribute to AFC. Am J Physiol Lung Cell Mol Physiol (2017) 312(6):L797-811. doi:10.1152/ ajplung.00379.2016

104. Kamynina E, Staub O. Concerted action of ENaC, Nedd4-2, and Sgk1 in transepithelial $\mathrm{Na}(+)$ transport. Am JPhysiol Renal Physiol (2002) 283(3):F377-87. doi:10.1152/ajprenal.00143.2002

105. Roux J, McNicholas CM, Carles M, Goolaerts A, Houseman BT, Dickinson DA, et al. IL-8 inhibits cAMP-stimulated alveolar epithelial fluid transport via a GRK2/PI3K-dependent mechanism. FASEB J (2013) 27(3):1095-106. doi:10.1096/fj.12-219295

106. Planes C, Leyvraz C, Uchida T, Angelova MA, Vuagniaux G, Hummler E, et al. In vitro and in vivo regulation of transepithelial lung alveolar sodium transport by serine proteases. Am J Physiol Lung Cell Mol Physiol (2005) 288(6):L1099-109. doi:10.1152/ajplung.00332.2004

107. Kleyman TR, Myerburg MM, Hughey RP. Regulation of ENaCs by proteases: an increasingly complex story. Kidney Int (2006) 70(8):1391-2. doi:10.1038/ sj.ki.5001860

108. Snyder PM. Minireview: regulation of epithelial Na+ channel trafficking. Endocrinology (2005) 146(12):5079-85. doi:10.1210/en.2005-0894

109. Dagenais A, Denis C, Vives MF, Girouard S, Masse C, Nguyen T, et al. Modulation of alpha-ENaC and alphal-Na+-K+-ATPase by cAMP and dexamethasone in alveolar epithelial cells. Am JPhysiol Lung Cell Mol Physiol (2001) 281(1):L217-30.

110. Kunzelmann K, Bachhuber T, Regeer R, Markovich D, Sun J, Schreiber R. Purinergic inhibition of the epithelial $\mathrm{Na}+$ transport via hydrolysis of PIP2. FASEB J (2005) 19(1):142-3. doi:10.1096/ff.04-2314fje

111. Helms MN, Jain L, Self JL, Eaton DC. Redox regulation of epithelial sodium channels examined in alveolar type 1 and 2 cells patch-clamped in lung slice tissue. J Biol Chem (2008) 283(33):22875-83. doi:10.1074/jbc.M801363200

112. Song W, Liu G, Bosworth CA, Walker JR, Megaw GA, Lazrak A, et al. Respiratory syncytial virus inhibits lung epithelial $\mathrm{Na}+$ channels by upregulating inducible nitric-oxide synthase. J Biol Chem (2009) 284(11): 7294-306. doi:10.1074/jbc.M806816200

113. Roux J, Kawakatsu H, Gartland B, Pespeni M, Sheppard D, Matthay MA, et al. Interleukin-1 beta decreases expression of the epithelial sodium channel alpha-subunit in alveolar epithelial cells via a p38 MAPK-dependent signaling pathway. J Biol Chem (2005) 280(19):18579-89. doi:10.1074/ jbc.M410561200

114. Shabbir W, Tzotzos S, Bedak M, Aufy M, Willam A, Kraihammer M, et al. Glycosylation-dependent activation of epithelial sodium channel by solnatide. Biochem Pharmacol (2015) 98(4):740-53. doi:10.1016/j.bcp.2015.08.003

115. Astrand AB, Hemmerling M, Root J, Wingren C, Pesic J, Johansson E, et al. Linking increased airway hydration, ciliary beating, and mucociliary clearance through $\mathrm{ENaC}$ inhibition. Am J Physiol Lung Cell Mol Physiol (2015) 308(1):L22-32. doi:10.1152/ajplung.00163.2014

116. Mall MA, Galietta LJ. Targeting ion channels in cystic fibrosis. J Cyst Fibros (2015) 14(5):561-70. doi:10.1016/j.jcf.2015.06.002

117. Matalon S, Bartoszewski R, Collawn JF. Role of epithelial sodium channels in the regulation of lung fluid homeostasis. Am J Physiol Lung Cell Mol Physiol (2015) 309(11):L1229-38. doi:10.1152/ajplung.00319.2015

118. Willam A, Aufy M, Tzotzos S, Evanzin H, Chytracek S, Geppert S, et al. Restoration of epithelial sodium channel function by synthetic peptides in pseudohypoaldosteronism type 1B mutants. Front Pharmacol (2017) 8:85. doi:10.3389/fphar.2017.00085

119. Shimkets RA, Warnock DG, Bositis CM, Nelson-Williams C, Hansson JH, Schambelan M, et al. Liddle's syndrome: heritable human hypertension caused by mutations in the beta subunit of the epithelial sodium channel. Cell (1994) 79(3):407-14. doi:10.1016/0092-8674(94)90250-X

120. Hansson JH, Schild L, Lu Y, Wilson TA, Gautschi I, Shimkets R, et al. A de novo missense mutation of the beta subunit of the epithelial sodium channel causes hypertension and Liddle syndrome, identifying a proline-rich segment critical for regulation of channel activity. Proc Natl Acad Sci U S A (1995) 92(25):11495-9. doi:10.1073/pnas.92.25.11495

121. Schild L, Canessa CM, Shimkets RA, Gautschi I, Lifton RP, Rossier BC. A mutation in the epithelial sodium channel causing Liddle disease increases channel activity in the Xenopus laevis oocyte expression system. Proc Natl Acad Sci U S A (1995) 92(12):5699-703. doi:10.1073/pnas.92.12.5699

122. Snyder PM, Price MP, McDonald FJ, Adams CM, Volk KA, Zeiher BG, et al. Mechanism by which Liddle's syndrome mutations increase activity of a human epithelial Na+ channel. Cell (1995) 83(6):969-78. doi:10.1016/00928674(95)90212-0

123. Inoue J, Iwaoka $\mathrm{T}$, Tokunaga $\mathrm{H}$, Takamune $\mathrm{K}$, Naomi $\mathrm{S}$, Araki $\mathrm{M}$, et al. A family with Liddle's syndrome caused by a new missense mutation in the beta subunit of the epithelial sodium channel. JClin Endocrinol Metab (1998) 83(6):2210-3. doi:10.1210/jcem.83.6.5030

124. Chang SS, Grunder S, Hanukoglu A, Rosler A, Mathew PM, Hanukoglu I, et al. Mutations in subunits of the epithelial sodium channel cause salt wasting with hyperkalaemic acidosis, pseudohypoaldosteronism type 1. Nat Genet (1996) 12(3):248-53. doi:10.1038/ng0396-248

125. Strautnieks SS, Thompson RJ, Gardiner RM, Chung E. A novel splice-site mutation in the gamma subunit of the epithelial sodium channel gene in three pseudohypoaldosteronism type 1 families. Nat Genet (1996) 13(2):248-50. doi:10.1038/ng0696-248

126. Gründer S, Firsov D, Chang SS, Jaeger NF, Gautschi I, Schild L, et al. A mutation causing pseudohypoaldosteronism type 1 identifies a conserved glycine that is involved in the gating of the epithelial sodium channel. EMBO $J$ (1997) 16(5):899-907. doi:10.1093/emboj/16.5.899

127. Boiko N, Kucher V, Stockand JD. Pseudohypoaldosteronism type 1 and Liddle's syndrome mutations that affect the single-channel properties of the epithelial Na+ channel. Physiol Rep (2015) 3(11):e12600. doi:10.14814/ phy 2.12600

128. Zennaro M-C, Lombès M. Mineralocorticoid resistance. Trends Endocrinol Metab (2004) 15(6):264-70. doi:10.1016/j.tem.2004.06.003

129. Riepe FG, van Bemmelen MX, Cachat F, Plendl H, Gautschi I, Krone N, et al. Revealing a subclinical salt-losing phenotype in heterozygous carriers of the novel S562P mutation in the alpha subunit of the epithelial sodium channel. Clin Endocrinol (Oxf) (2009) 70(2):252-8. doi:10.1111/j.13652265.2008.03314.x

130. Eisenhut M, Wallace H. Ion channels in inflammation. Pflugers Arch (2011) 461(4):401-21. doi:10.1007/s00424-010-0917-y

131. Meltzer RH, Kapoor N, Qadri YJ, Anderson SJ, Fuller CM, Benos DJ. Heteromeric assembly of acid-sensitive ion channel and epithelial sodium channel subunits. J Biol Chem (2007) 282(35):25548-59. doi:10.1074/jbc. M703825200 
132. Frank J, Roux J, Kawakatsu H, Su G, Dagenais A, Berthiaume Y, et al. Transforming growth factor-betal decreases expression of the epithelial sodium channel alphaENaC and alveolar epithelial vectorial sodium and fluid transport via an ERK1/2-dependent mechanism. J Biol Chem (2003) 278(45):43939-50. doi:10.1074/jbc.M304882200

133. Peters DM, Vadász I, Wujak Ł, Wygrecka M, Olschewski A, Becker C, et al. TGF- $\beta$ directs trafficking of the epithelial sodium channel ENaC which has implications for ion and fluid transport in acute lung injury. Proc Natl Acad Sci U S A (2014) 111(3):E374-83. doi:10.1073/ pnas.1306798111

134. Willis BC, Kim KJ, Li X, Liebler J, Crandall ED, Borok Z. Modulation of ion conductance and active transport by TGF-beta 1 in alveolar epithelial cell monolayers. Am JPhysiol Lung Cell Mol Physiol (2003) 285(6): L1192-200. doi:10.1152/ajplung.00379.2002

135. Dagenais A, Fréchette R, Yamagata Y, Yamagata T, Carmel J-F, Clermont M-E, et al. Downregulation of ENaC activity and expression by TNF- $\alpha$ in alveolar epithelial cells. Am J Physiol Lung Cell Mol Physiol (2004) 286(2):L301-11. doi:10.1152/ajplung.00326.2002

136. Fukuda N, Jayr C, Lazrak A, Wang Y, Lucas R, Matalon S, et al. Mechanisms of TNF-alpha stimulation of amiloride-sensitive sodium transport across alveolar epithelium. Am J Physiol Lung Cell Mol Physiol (2001) 280(6):1258-65.

137. Choi JY, Choi YS, Kim SJ, Son EJ, Choi HS, Yoon JH. Interleukin-1beta suppresses epithelial sodium channel beta-subunit expression and ENaCdependent fluid absorption in human middle ear epithelial cells. Eur J Pharmacol (2007) 567(1-2):19-25. doi:10.1016/j.ejphar.2007.04.026

138. Gray T, Coakley R, Hirsh A, Thornton D, Kirkham S, Koo JS, et al. Regulation of MUC5AC mucin secretion and airway surface liquid metabolism by IL-1 $\beta$ in human bronchial epithelia. Am J Physiol Lung Cell Mol Physiol (2004) 286:L320-30. doi:10.1152/ajplung.00440.2002

139. Geiser T, Jarreau PH, Atabai K, Matthay MA. Interleukin-1beta augments in vitro alveolar epithelial repair. Am J Physiol Lung Cell Mol Physiol (2000) 279(6):L1184-90.

140. Nair PD, Li T, Bhattacharjee R, Ye X, Folkesson HG. Oxytocin-induced labor augments IL-1 beta-stimulated lung fluid absorption in fetal guinea pig lungs. Am J Physiol Lung Cell Mol Physiol (2005) 289(6):L1029-38. doi:10.1152/ajplung.00256.2004

141. Galietta LJV, Pagesy P, Folli C, Caci E, Romio L, Costes B, et al. IL-4 is a potent modulator of ion transport in the human bronchial epithelium in vitro. J Immunol (2002) 168(2):839. doi:10.4049/jimmunol.168.2.839

142. Galietta LJ, Folli C, Caci E, Pedemonte N, Taddei A, Ravazzolo R, et al. Effect of inflammatory stimuli on airway ion transport. Proc Am Thorac Soc (2004) 1(1):62-5. doi:10.1513/pats.2306017

143. Zhou L, Graeff RW, McCray PB Jr., Simonet WS, Whitsett JA. Keratinocyte growth factor stimulates CFTR-independent fluid secretion in the fetal lung in vitro. Am J Physiol (1996) 271(6 Pt 1):L987-94.

144. Ling B, DC E. Effects of luminal $\mathrm{Na}+$ on single $\mathrm{Na}+$ channels in A6 cells, a regulatory role for protein kinase C. Am J Physiol Renal Fluid Electrolyte Physiol (1989) 256:F1094-103.

145. Frindt G, Palmer LG, Windhager EE. Feedback regulation of Na channels in rat CCT. IV. Mediation by activation of protein kinase C. Am J Physiol (1996) 270(2 Pt 2):F371-6.

146. Stockand JD, Bao HF, Schenck J, Malik B, Middleton P, Schlanger LE, et al. Differential effects of protein kinase $\mathrm{C}$ on the levels of epithelial $\mathrm{Na}+$ channel subunit proteins. J Biol Chem (2000) 275(33):25760-5. doi:10.1074/jbc. M003615200

147. Soukup B, Benjamin A, Orogo-Wenn M, Walters D. Physiological effect of protein kinase $\mathrm{C}$ on $\mathrm{ENaC}$-mediated lung liquid regulation in the adult rat lung. Am J Physiol Lung Cell Mol Physiol (2012) 302(1):L133-9. doi:10.1152/ ajplung.00031.2011

148. Migneault F, Boncoeur E, Morneau F, Pascariu M, Dagenais A, Berthiaume Y. Cycloheximide and lipopolysaccharide downregulate alphaENaC mRNA via different mechanisms in alveolar epithelial cells. Am J Physiol Lung Cell Mol Physiol (2013) 305(10):L747-55. doi:10.1152/ajplung.00023.2013

149. Lucas R, Sridhar S, Rick FG, Gorshkov B, Umapathy NS, Yang G, et al. Agonist of growth hormone-releasing hormone reduces pneumolysininduced pulmonary permeability edema. Proc Natl Acad Sci U S A (2012) 109(6):2084-9. doi:10.1073/pnas.1121075109

150. Downs CA, Helms MN. Regulation of ion transport by oxidants. Am J Physiol Lung Cell Mol Physiol (2013) 305(9):L595. doi:10.1152/ajplung.00212.2013
151. Downs CA, Kreiner L, Zhao XM, Trac P, Johnson NM, Hansen JM, et al. Oxidized glutathione (GSSG) inhibits epithelial sodium channel activity in primary alveolar epithelial cells. Am J Physiol Lung Cell Mol Physiol (2015) 308(9):L943-52. doi:10.1152/ajplung.00213.2014

152. Zhu S, Ware LB, Geiser T, Matthay MA, Matalon S. Increased levels of nitrate and surfactant protein a nitration in the pulmonary edema fluid of patients with acute lung injury. Am J Respir Crit Care Med (2001) 163(1):166-72. doi:10.1164/ajrccm.163.1.2005068

153. Snyder PM. Intoxicated $\mathrm{Na}(+)$ channels. Focus on "Ethanol stimulates epithelial sodium channels by elevating reactive oxygen species". Am J Physiol Cell Physiol (2012) 303(11):C1125-6. doi:10.1152/ajpcell.00301.2012

154. Lazrak A, Jurkuvenaite A, Ness EC, Zhang S, Woodworth BA, Muhlebach MS, et al. Inter- $\alpha$-inhibitor blocks epithelial sodium channel activation and decreases nasal potential differences in $\Delta$ F508 mice. Am J Respir Cell Mol Biol (2014) 50(5):953-62. doi:10.1165/rcmb.2013-0215OC

155. Zhou R, Patel SV, Snyder PM. Nedd4-2 catalyzes ubiquitination and degradation of cell surface ENaC. J Biol Chem (2007) 282(28):20207-12. doi:10.1074/jbc.M611329200

156. Planes C, Blot-Chabaud M, Matthay MA, Couette S, Uchida T, Clerici C. Hypoxia and beta 2-agonists regulate cell surface expression of the epithelial sodium channel in native alveolar epithelial cells. J Biol Chem (2002) 277(49):47318-24. doi:10.1074/jbc.M209158200

157. Poulsen AN, Klausen TL, Pedersen PS, Willumsen NJ, Frederiksen O. Regulation of ion transport via apical purinergic receptors in intact rabbit airway epithelium. Pflugers Arch (2005) 450(4):227-35. doi:10.1007/s00424005-1388-4

158. Burnstock G, Brouns I, Adriaensen D, Timmermans J-P. Purinergic signaling in the airways. Pharmacol Rev (2012) 64(4):834. doi:10.1124/pr.111.005389

159. Takemura Y, Helms MN, Eaton AF, Self J, Ramosevac S, Jain L, et al. Cholinergic regulation of epithelial sodium channels in rat alveolar type 2 epithelial cells. Am J Physiol Lung Cell Mol Physiol (2013) 304(6):L428-37. doi:10.1152/ajplung.00129.2012

160. Greenlee MM, Mitzelfelt JD, Yu L, Yue Q, Duke BJ, Harrell CS, et al. Estradiol activates epithelial sodium channels in rat alveolar cells through the G protein-coupled estrogen receptor. Am J Physiol Lung Cell Mol Physiol (2013) 305(11):L878-89. doi:10.1152/ajplung.00008.2013

161. Lazrak A, Nielsen VG, Matalon S. Mechanisms of increased $\mathrm{Na}(+)$ transport in ATII cells by cAMP: we agree to disagree and do more experiments. Am J Physiol Lung Cell Mol Physiol (2000) 278(2):L233-8.

162. Itani OA, Liu KZ, Cornish KL, Campbell JR, Thomas CP. Glucocorticoids stimulate human sgk1 gene expression by activation of a GRE in its 5'-flanking region. Am J Physiol Endocrinol Metab (2002) 283(5):E971-9. doi:10.1152/ ajpendo.00021.2002

163. Dagenais A, Gosselin D, Guilbault C, Radzioch D, Berthiaume Y. Modulation of epithelial sodium channel $(\mathrm{ENaC})$ expression in mouse lung infected with Pseudomonas aeruginosa. Respir Res (2005) 6:2. doi:10.1186/ 1465-9921-6-2

164. Dagenais A, Frechette R, Clermont ME, Masse C, Prive A, Brochiero E, et al. Dexamethasone inhibits the action of TNF on ENaC expression and activity. Am J Physiol Lung Cell Mol Physiol (2006) 291(6):L1220-31. doi:10.1152/ajplung.00511.2005

165. Brodovich H, Canessa C, Ueda J, Rafii B, Rossier BC, Edelson J. Expression of the epithelial $\mathrm{Na}+$ channel in the developing rat lung. Am J Physiol (1993) 265(2):C491.

166. Tchepichev S, Ueda J, Canessa C, Rossier BC, Brodovich H. Lung epithelial $\mathrm{Na}$ channel subunits are differentially regulated during development and by steroids. Am J Physiol Cell Physiol (1995) 269(3):C805.

167. Husted RF, Volk KA, Sigmund RD, Stokes JB. Discordant effects of corticosteroids and expression of subunits on ENaC activity. Am J Physiol Renal Physiol (2007) 293(3):F813-20. doi:10.1152/ajprenal.00225.2007

168. Aggarwal S, Dabla PK, Arora S. Prostasin: an epithelial sodium channel regulator. J Biomark (2013) 2013:9. doi:10.1155/2013/179864

169. Bruns JB, Carattino MD, Sheng S, Maarouf AB, Weisz OA, Pilewski JM, et al. Epithelial $\mathrm{Na}+$ channels are fully activated by furin- and prostasindependent release of an inhibitory peptide from the gamma-subunit. J Biol Chem (2007) 282(9):6153-60. doi:10.1074/jbc.M610636200

170. Booth RE, Stockand JD. Targeted degradation of ENaC in response to PKC activation of the ERK1/2 cascade. Am J Physiol Renal Physiol (2003) 284(5):F938-47. doi:10.1152/ajprenal.00373.2002 
171. Hughey RP, Mueller GM, Bruns JB, Kinlough CL, Poland PA, Harkleroad KL, et al. Maturation of the epithelial $\mathrm{Na}+$ channel involves proteolytic processing of the alpha- and gamma-subunits. JBiol Chem (2003) 278(39): 37073-82. doi:10.1074/jbc.M307003200

172. Sheng S, Carattino MD, Bruns JB, Hughey RP, Kleyman TR. Furin cleavage activates the epithelial $\mathrm{Na}+$ channel by relieving $\mathrm{Na}+$ self-inhibition. Am JPhysiol Renal Physiol (2006) 290(6):F1488-96. doi:10.1152/ajprenal. 00439.2005

173. Passero CJ, Mueller GM, Rondon-Berrios H, Tofovic SP, Hughey RP, Kleyman TR. Plasmin activates epithelial $\mathrm{Na}(+)$ channels by cleaving the $\gamma$ subunit. JBiol Chem (2008) 283(52):36586-91. doi:10.1074/jbc. M805676200

174. Eaton DC, Chen J, Ramosevac S, Matalon S, Jain L. Regulation of Na+ channels in lung alveolar type II epithelial cells. Proc Am Thorac Soc (2004) 1(1):10-6. doi:10.1513/pats.2306008

175. Kunzelmann $\mathrm{K}$. $\mathrm{ENaC}$ is inhibited by an increase in the intracellular $\mathrm{Cl}(-)$ concentration mediated through activation of $\mathrm{Cl}(-)$ channels. Pflugers Arch (2003) 445(4):504-12. doi:10.1007/s00424-002-0958-y

176. Helms MN, Chen XJ, Ramosevac S, Eaton DC, Jain L. Dopamine regulation of amiloride-sensitive sodium channels in lung cells. Am J Physiol Lung Cell Mol Physiol (2006) 290(4):L710-22. doi:10.1152/ajplung.00486.2004

177. Downs CA, Kreiner LH, Johnson NM, Brown LA, Helms MN. Receptor for advanced glycation end-products regulates lung fluid balance via protein kinase C-gp91(phox) signaling to epithelial sodium channels. Am J Respir Cell Mol Biol (2015) 52(1):75-87. doi:10.1165/rcmb.2014-0002OC

178. Besancon F, Przewlocki G, Baro I, Hongre AS, Escande D, Edelman A. Interferon-gamma downregulates CFTR gene expression in epithelial cells. Am J Physiol (1994) 267(5 Pt 1):C1398-404.

179. Pruliere-Escabasse V, Fanen P, Dazy AC, Lechapt-Zalcman E, Rideau D, Edelman A, et al. TGF-beta 1 downregulates CFTR expression and function in nasal polyps of non-CF patients. Am J Physiol Lung Cell Mol Physiol (2005) 288(1):L77-83. doi:10.1152/ajplung.00048.2004

180. Danahay H, Atherton H, Jones G, Bridges RJ, Poll CT. Interleukin-13 induces a hypersecretory ion transport phenotype in human bronchial epithelial cells. Am J Physiol Lung Cell Mol Physiol (2002) 282(2):L226-36. doi:10.1152/ajplung.00311.2001

181. Cafferata EG, Gonzalez-Guerrico AM, Giordano L, Pivetta OH, SantaColoma TA. Interleukin-1beta regulates CFTR expression in human intestinal T84 cells. Biochim Biophys Acta (2000) 1500(2):241-8. doi:10.1016/ S0925-4439(99)00105-2

182. Jiang X, Ingbar DH, O'Grady SM. Adrenergic stimulation of $\mathrm{Na}+$ transport across alveolar epithelial cells involves activation of apical Cl- channels. Am J Physiol (1998) 275(6 Pt 1):C1610-20.

183. Fang X, Fukuda N, Barbry P, Sartori C, Verkman AS, Matthay MA. Novel role for CFTR in fluid absorption from the distal airspaces of the lung. J Gen Physiol (2002) 119(2):199-207. doi:10.1085/jgp.119.2.199

184. Trotta T, Guerra L, Piro D, d'Apolito M, Piccoli C, Porro C, et al. Stimulation of $\beta 2$-adrenergic receptor increases CFTR function and decreases ATP levels in murine hematopoietic stem/progenitor cells. J Cyst Fibros (2015) 14(1):26-33. doi:10.1016/j.jcf.2014.08.005

185. Baudouin-Legros M, Brouillard F, Tondelier D, Hinzpeter A, Edelman A. Effect of ouabain on CFTR gene expression in human Calu-3 cells. Am J Physiol Cell Physiol (2003) 284(3):C620-6. doi:10.1152/ajpcell.00457.2002

186. Welsh MJ, Smith JJ. cAMP stimulation of $\mathrm{HCO} 3-$ secretion across airway epithelia. JOP (2001) 2(4 Suppl):291-3.

187. Qiu W, Laheri A, Leung S, Guggino SE. Hormones increase mRNA of cyclic-nucleotide-gated cation channels in airway epithelia. Pflugers Arch (2000) 441(1):69-77. doi:10.1007/s004240000359

188. Ousingsawat J, Kongsuphol P, Schreiber R, Kunzelmann K. CFTR and TMEM16A are separate but functionally related Cl- channels. Cell Physiol Biochem (2011) 28(4):715-24. doi:10.1159/000335765

189. Zhou Y, Dong Q, Louahed J, Dragwa C, Savio D, Huang M, et al. Characterization of a calcium-activated chloride channel as a shared target of Th2 cytokine pathways and its potential involvement in asthma. Am J Respir Cell Mol Biol (2001) 25(4):486-91. doi:10.1165/ajrcmb.25.4.4578

190. Towne JE, Krane CM, Bachurski CJ, Menon AG. Tumor necrosis factoralpha inhibits aquaporin 5 expression in mouse lung epithelial cells. J Biol Chem (2001) 276(22):18657-64. doi:10.1074/jbc.M100322200
191. Sidhaye VK, Guler AD, Schweitzer KS, D’Alessio F, Caterina MJ, King LS. Transient receptor potential vanilloid 4 regulates aquaporin-5 abundance under hypotonic conditions. Proc Natl Acad Sci U S A (2006) 103(2):4747-52. doi:10.1073/pnas.0511211103

192. Chu S, Blaisdell CJ, Bamford P, Ferro TJ. Interferon-gamma regulates ClC-2 chloride channel in lung epithelial cells. Biochem Biophys Res Commun (2004) 324(1):31-9. doi:10.1016/j.bbrc.2004.09.026

193. Yang F, Kawedia JD, Menon AG. Cyclic AMP regulates aquaporin 5 expression at both transcriptional and post-transcriptional levels through a protein kinase A pathway. J Biol Chem (2003) 278(34):32173-80. doi:10.1074/jbc. M305149200

194. Csanyi A, Bota J, Falkay G, Gaspar R, Ducza E. The effects of female sexual hormones on the expression of aquaporin 5 in the late-pregnant rat uterus. Int J Mol Sci (2016) 17(8):E1300. doi:10.3390/ijms17081300

195. Sugi K, Musch MW, Field M, Chang EB. Inhibition of Na+,K+-ATPase by interferon gamma down-regulates intestinal epithelial transport and barrier function. Gastroenterology (2001) 120(6):1393-403. doi:10.1053/ gast.2001.24045

196. Vadasz I, Schermuly RT, Ghofrani HA, Rummel S, Wehner S, Muhldorfer I, et al. The lectin-like domain of tumor necrosis factor-alpha improves alveolar fluid balance in injured isolated rabbit lungs. Crit Care Med (2008) 36(5):1543-50. doi:10.1097/CCM.0b013e31816f485e

197. Wujak LA, Becker S, Seeger W, Morty RE. TGF- $\beta$ regulates Na,K-ATPase activity by changing the regulatory subunit stoichiometry of the $\mathrm{Na}, \mathrm{K}$ ATPase complex. FASEB J (2011) 25(1 Suppl):1039.

198. Wujak LA, Blume A, Baloglu E, Wygrecka M, Wygowski J, Herold S, et al. FXYD1 negatively regulates $\mathrm{Na}(+) / \mathrm{K}(+)$-ATPase activity in lung alveolar epithelial cells. Respir Physiol Neurobiol (2016) 220:54-61. doi:10.1016/ j.resp.2015.09.008

199. Peteranderl C, Morales-Nebreda L, Selvakumar B, Lecuona E, Vadasz I, Morty RE, et al. Macrophage-epithelial paracrine crosstalk inhibits lung edema clearance during influenza infection. JClin Invest (2016) 126(4):1566-80. doi:10.1172/JCI83931

200. Sloniewsky DE, Ridge KM, Adir Y, Fries FP, Briva A, Sznajder JI, et al. Leukotriene D4 activates alveolar epithelial Na,K-ATPase and increases alveolar fluid clearance. Am J Respir Crit Care Med (2004) 169(3):407-12. doi:10.1164/rccm.200304-472OC

201. Li X, Yan XX, Li HL, Li RQ. Endogenous acetylcholine increases alveolar epithelial fluid transport via activation of alveolar epithelial $\mathrm{Na}, \mathrm{K}$-ATPase in mice. Respir Physiol Neurobiol (2015) 217:25-31. doi:10.1016/j.resp. 2015.05.005

202. Pittet JF, Lu M, Morris DG, Modelska K, Welch WJ, Carey HV, et al. Reactive nitrogen species inhibit alveolar epithelial fluid transport after hemorrhagic shock in rats. J Immunol (2001) 166. doi:10.4049/jimmunol.166.10.6301

203. Bechara RI, Brown LA, Roman J, Joshi PC, Guidot DM. Transforming growth factor betal expression and activation is increased in the alcoholic rat lung. Am J Respir Crit Care Med (2004) 170(2):188-94. doi:10.1164/ rccm.200304-478OC

204. Bechara RI, Pelaez A, Palacio A, Joshi PC, Hart CM, Brown LA, et al. Angiotensin II mediates glutathione depletion, transforming growth factorbetal expression, and epithelial barrier dysfunction in the alcoholic rat lung. Am J Physiol Lung Cell Mol Physiol (2005) 289(3):L363-70. doi:10.1152/ ajplung.00141.2005

205. Sheppard D. Transforming growth factor beta: a central modulator of pulmonary and airway inflammation and fibrosis. Proc Am Thorac Soc (2006) 3(5):413-7. doi:10.1513/pats.200601-008AW

206. Pittet JF, Griffiths MJ, Geiser T, Kaminski N, Dalton SL, Huang X, et al. TGF-beta is a critical mediator of acute lung injury. JClin Invest (2001) 107(12):1537-44. doi:10.1172/jci11963

207. Patel BV, Wilson MR, O’Dea KP, Takata M. TNF-induced death signaling triggers alveolar epithelial dysfunction in acute lung injury. JImmunol (2013) 190(8):4274-82. doi:10.4049/jimmunol.1202437

208. Hogner K, Wolff T, Pleschka S, Plog S, Gruber AD, Kalinke U, et al. Macrophage-expressed IFN-beta contributes to apoptotic alveolar epithelial cell injury in severe influenza virus pneumonia. PLoS Pathog (2013) 9(2):e1003188. doi:10.1371/journal.ppat.1003188

209. Peteranderl C, Herold S. The impact of the interferon/TNF-related apoptosis-inducing ligand signaling axis on disease progression in 
respiratory viral infection and beyond. Front Immunol (2017) 8:313. doi:10.3389/fimmu.2017.00313

210. Gan H, Wang G, Hao Q, Wang QJ, Tang H. Protein kinase D promotes airway epithelial barrier dysfunction and permeability through downregulation of claudin-1. J Biol Chem (2014) 289(30):20489. doi:10.1074/jbc. A113.511527

211. Mitchell LA, Overgaard CE, Ward C, Margulies SS, Koval M. Differential effects of claudin-3 and claudin-4 on alveolar epithelial barrier function. Am J Physiol Lung Cell Mol Physiol (2011) 301(1):L40-9. doi:10.1152/ ajplung.00299.2010

212. Wray C, Mao Y, Pan J, Chandrasena A, Piasta F, Frank JA. Claudin-4 augments alveolar epithelial barrier function and is induced in acute lung injury. Am J Physiol Lung Cell Mol Physiol (2009) 297(2):L219-27. doi:10.1152/ ajplung.00043.2009

213. Alvarez DF, King JA, Weber D, Addison E, Liedtke W, Townsley MI. Transient receptor potential vanilloid 4-mediated disruption of the alveolar septal barrier: a novel mechanism of acute lung injury. Circ Res (2006) 99(9):988-95. doi:10.1161/01.res.0000247065.11756.19

214. Curry-McCoy TV, Venado A, Guidot DM, Joshi PC. Alcohol ingestion disrupts alveolar epithelial barrier function by activation of macrophagederived transforming growth factor betal. Respir Res (2013) 14:39. doi:10.1186/1465-9921-14-39

215. Gerloff J, Sundar IK, Freter R, Sekera ER, Friedman AE, Robinson R, et al. Inflammatory response and barrier dysfunction by different e-cigarette flavoring chemicals identified by gas chromatography-mass spectrometry in e-liquids and e-vapors on human lung epithelial cells and fibroblasts. Appl In Vitro Toxicol (2017) 3(1):28-40. doi:10.1089/aivt.2016.0030

216. Peterson MW, Walter ME, Gross TJ. Asbestos directly increases lung epithelial permeability. Am J Physiol Lung Cell Mol Physiol (1993) 265(3):L308.

217. Statt S, Ruan JW, Hung LY, Chang CY, Huang CT, Lim JH, et al. Statinconferred enhanced cellular resistance against bacterial pore-forming toxins in airway epithelial cells. Am J Respir Cell Mol Biol (2015) 53(5): 689-702. doi:10.1165/rcmb.2014-03910C

218. Albertine KH, Soulier MF, Wang Z, Ishizaka A, Hashimoto S, Zimmerman GA, et al. Fas and Fas ligand are up-regulated in pulmonary edema fluid and lung tissue of patients with acute lung injury and the acute respiratory distress syndrome. Am J Pathol (2002) 161(5):1783-96. doi:10.1016/S0002-9440(10)64455-0

219. Herrero R, Tanino M, Smith LS, Kajikawa O, Wong VA, Mongovin S, et al. The Fas/FasL pathway impairs the alveolar fluid clearance in mouse lungs. Am J Physiol Lung Cell Mol Physiol (2013) 305(5):L377-88. doi:10.1152/ ajplung.00271.2012

220. Fein A, Grossman RF, Jones JG, Hoeffel J, McKay D. Carbon monoxide effect on alveolar epithelial permeability. Chest (1980) 78(5):726-31. doi:10.1378/chest.78.5.726

221. Wilson MR, O’Dea KP, Dorr AD, Yamamoto H, Goddard ME, Takata M. Efficacy and safety of inhaled carbon monoxide during pulmonary inflammation in mice. PLoS One (2010) 5(7):e11565. doi:10.1371/journal. pone. 0011565

222. Kolosova IA, Mirzapoiazova T, Moreno-Vinasco L, Sammani S, Garcia JG, Verin AD. Protective effect of purinergic agonist ATPgammaS against acute lung injury. Am J Physiol Lung Cell Mol Physiol (2008) 294(2):L319-24. doi:10.1152/ajplung.00283.2007

223. Rajasekaran AK, Rajasekaran SA. Role of Na-K-ATPase in the assembly of tight junctions. Am J Physiol Ren Physiol (2003) 285(3):F388. doi:10.1152/ ajprenal.00439.2002

224. Lin X, Barravecchia M, Kothari P, Young JL, Dean DA. [beta]1-Na+,K+ATPase gene therapy upregulates tight junctions to rescue lipopolysaccharideinduced acute lung injury. Gene Ther (2016) 23(6):489-99. doi:10.1038/ gt.2016.19

225. Han X, Fink MP, Uchiyama T, Delude RL. Increased iNOS activity is essential for pulmonary epithelial tight junction dysfunction in endotoxemic mice. Am J Physiol Lung Cell Mol Physiol (2003) 286(2):L259-67. doi:10.1152/ ajplung.00187.2003

226. Short KR, Kasper J, van der Aa S, Andeweg AC, Zaaraoui-Boutahar F, Goeijenbier $M$, et al. Influenza virus damages the alveolar barrier by disrupting epithelial cell tight junctions. Eur Respir J (2016) 47(3):954. doi:10.1183/13993003.01282-2015
227. Xu S, Xue X, You K, Fu J. Caveolin-1 regulates the expression of tight junction proteins during hyperoxia-induced pulmonary epithelial barrier breakdown. Respir Res (2016) 17(1):50. doi:10.1186/s12931-016-0364-1

228. Petecchia L, Sabatini F, Usai C, Caci E, Varesio L, Rossi GA. Cytokines induce tight junction disassembly in airway cells via an EGFR-dependent MAPK/ERK1/2-pathway. Lab Invest (2012) 92(8):1140-8. doi:10.1038/ labinvest.2012.67

229. Saatian B, Rezaee F, Desando S, Emo J, Chapman T, Knowlden S, et al. Interleukin-4 and interleukin-13 cause barrier dysfunction in human airway epithelial cells. Tissue Barriers (2013) 1(2):e24333. doi:10.4161/tisb.24333

230. Mazzon E, Cuzzocrea S. Role of TNF-alpha in lung tight junction alteration in mouse model of acute lung inflammation. Respir Res (2007) 8:75. doi:10.1186/1465-9921-8-75

231. Youakim A, Ahdieh M. Interferon-gamma decreases barrier function in T84 cells by reducing ZO-1 levels and disrupting apical actin. Am J Physiol (1999) 276(5 Pt 1):G1279-88.

232. Ahdieh M, Vandenbos T, Youakim A. Lung epithelial barrier function and wound healing are decreased by IL- 4 and IL-13 and enhanced by IFNgamma. Am J Physiol Cell Physiol (2001) 281(6):C2029-38.

233. Petecchia L, Sabatini F, Varesio L, Camoirano A, Usai C, Pezzolo A, et al. Bronchial airway epithelial cell damage following exposure to cigarette smoke includes disassembly of tight junction components mediated by the extracellular signal-regulated kinase 1/2 pathway. Chest (2009) 135(6):1502-12. doi:10.1378/chest.08-1780

234. Cao X, Lin H, Muskhelishvili L, Latendresse J, Richter P, Heflich RH. Tight junction disruption by cadmium in an in vitro human airway tissue model. Respir Res (2015) 16:30. doi:10.1186/s12931-015-0191-9

235. Lu Q, Harrington EO, Jackson H, Morin N, Shannon C, Rounds S. Transforming growth factor-betal-induced endothelial barrier dysfunction involves Smad2-dependent p38 activation and subsequent RhoA activation. J Appl Physiol (1985) (2006) 101(2):375-84. doi:10.1152/japplphysiol. 01515.2005

236. van der Poll T, Lowry SF. Tumor necrosis factor in sepsis: mediator of multiple organ failure or essential part of host defense? Shock (1995) 3(1):1-12. doi:10.1097/00024382-199503010-00001

237. Tracey KJ, Fong Y, Hesse DG, Manogue KR, Lee AT, Kuo GC, et al. Anticachectin/TNF monoclonal antibodies prevent septic shock during lethal bacteraemia. Nature (1987) 330(6149):662-4. doi:10.1038/330662a0

238. Koss M, Pfeiffer GR II, Wang Y, Thomas ST, Yerukhimovich M, Gaarde WA, et al. Ezrin/radixin/moesin proteins are phosphorylated by TNF-alpha and modulate permeability increases in human pulmonary microvascular endothelial cells. J Immunol (2006) 176(2):1218-27. doi:10.4049/ jimmunol.176.2.1218

239. Lucas R, Garcia I, Donati YR, Hribar M, Mandriota SJ, Giroud C, et al. Both TNF receptors are required for direct TNF-mediated cytotoxicity in microvascular endothelial cells. Eur JImmunol (1998) 28(11):3577-86. doi:10.1002/(SICI)1521-4141(199811)28:11<3577::AID-IMMU3577>3.0. CO;2-\#

240. Stammberger U, Gaspert A, Hillinger S, Vogt P, Odermatt B, Weder W, et al. Apoptosis induced by ischemia and reperfusion in experimental lung transplantation. Ann Thorac Surg (2000) 69(5):1532-6. doi:10.1016/ S0003-4975(00)01228-5

241. Xiong C, Yang G, Kumar S, Aggarwal S, Leustik M, Snead C, et al. The lectin-like domain of TNF protects from listeriolysin-induced hyperpermeability in human pulmonary microvascular endothelial cells - a crucial role for protein kinase C-alpha inhibition. Vascul Pharmacol (2010) 52(5-6): 207-13. doi:10.1016/j.vph.2009.12.010

242. Hribar M, Bloc A, van der Goot FG, Fransen L, De Baetselier P, Grau GE, et al. The lectin-like domain of tumor necrosis factor-alpha increases membrane conductance in microvascular endothelial cells and peritoneal macrophages. Eur J Immunol (1999) 29(10):3105-11. doi:10.1002/ (SICI) 1521-4141(199910)29:10<3105::AID-IMMU3105>3.0.CO;2-A

243. Martin S, Maruta K, Burkart V, Gillis S, Kolb H. IL-1 and IFN-gamma increase vascular permeability. Immunology (1988) 64(2):301-5.

244. Leff JA, Bodman ME, Cho OJ, Rohrbach S, Reiss OK, Vannice JL, et al. Postinsult treatment with interleukin-1 receptor antagonist decreases oxidative lung injury in rats given intratracheal interleukin-1. Am J Respir Crit Care Med (1994) 150(1):109-12. doi:10.1164/ajrccm.150.1.8025734 
245. Repine JE. Interleukin-1-mediated acute lung injury and tolerance to oxidative injury. Environ Health Perspect (1994) 102(Suppl 10):75-8. doi:10.1289/ehp.94102s1075

246. Hybertson BM, Lee YM, Cho HG, Cho OJ, Repine JE. Alveolar type II cell abnormalities and peroxide formation in lungs of rats given IL-1 intratracheally. Inflammation (2000) 24(4):289-303. doi:10.1023/A:1007092 529261

247. Lee YM, Hybertson BM, Cho HG, Terada LS, Cho O, Repine AJ, et al. Plateletactivating factor contributes to acute lung leak in rats given interleukin-1 intratracheally. Am J Physiol Lung Cell Mol Physiol (2000) 279(1):L75-80.

248. Ballmer-Weber BK, Dummer R, Küng E, Burg G, Ballmer PE. Interleukin 2 -induced increase of vascular permeability without decrease of the intravascular albumin pool. Br J Cancer (1995) 71(1):78-82. doi:10.1038/bjc.1995.16

249. Maruo N, Morita I, Shirao M, Murota S. IL-6 increases endothelial permeability in vitro. Endocrinology (1992) 131(2):710-4. doi:10.1210/endo. 131.2.1639018

250. Biffl WL, Moore EE, Moore FA, Carl VS, Franciose RJ, Banerjee A. Interleukin-8 increases endothelial permeability independent of neutrophils. J Trauma (1995) 39(1):98-102. doi:10.1097/00005373-19950700000013; discussion 102-103,

251. Matsumoto K, Ohi H, Kanmatsuse K. Interleukin 12 upregulates the release of vascular permeability factor by peripheral blood mononuclear cells from patients with lipoid nephrosis. Nephron (1998) 78(4):403-9. doi:10.1159/000044968

252. DiStasi MR, Ley K. Opening the flood-gates: how neutrophil-endothelial interactions regulate permeability. Trends Immunol (2009) 30(11):547-56. doi:10.1016/j.it.2009.07.012

253. Thorneloe KS, Cheung M, Bao W, Alsaid H, Lenhard S, Jian MY, et al. An orally active TRPV4 channel blocker prevents and resolves pulmonary edema induced by heart failure. Sci Transl Med (2012) 4(159):159ra148. doi:10.1126/scitranslmed.3004276

254. Morty RE, Kuebler WM. TRPV4: an exciting new target to promote alveolocapillary barrier function. Am J Physiol Lung Cell Mol Physiol (2014) 307(11):L817-21. doi:10.1152/ajplung.00254.2014

255. Mehta D, Ahmmed GU, Paria BC, Holinstat M, Voyno-Yasenetskaya T, Tiruppathi C, et al. RhoA interaction with inositol 1,4,5-trisphosphate receptor and transient receptor potential channel-1 regulates Ca2+ entry. Role in signaling increased endothelial permeability. J Biol Chem (2003) 278(35):33492-500. doi:10.1074/jbc.M302401200

256. Lien DC, Worthen GS, Henson PM, Bethel RA. Platelet-activating factor causes neutrophil accumulation and neutrophil-mediated increased vascular permeability in canine trachea. Am Rev Respir Dis (1992) 145(3): 693-700. doi:10.1164/ajrccm/145.3.693

257. Lee KS, Kim SR, Park SJ, Park HS, Min KH, Lee MH, et al. Hydrogen peroxide induces vascular permeability via regulation of vascular endothelial growth factor. Am J Respir Cell Mol Biol (2006) 35(2):190-7. doi:10.1165/ rcmb.2005-0482OC

258. Su G, Hodnett M, Wu N, Atakilit A, Kosinski C, Godzich M, et al. Integrin $\alpha v \beta 5$ regulates lung vascular permeability and pulmonary endothelial barrier function. Am J Respir Cell Mol Biol (2007) 36(3):377-86. doi:10.1165/ $\mathrm{rcmb}$.2006-0238OC

259. Semina EV, Rubina KA, Sysoeva VY, Rutkevich PN, Kashirina NM, Tkachuk VA. Novel mechanism regulating endothelial permeability via T-cadherin-dependent VE-cadherin phosphorylation and clathrinmediated endocytosis. Mol Cell Biochem (2014) 387(1):39-53. doi:10.1007/ s11010-013-1867-4

260. Shen Q, Rigor RR, Pivetti CD, Wu MH, Yuan SY. Myosin light chain kinase in microvascular endothelial barrier function. Cardiovasc Res (2010) 87(2):272-80. doi:10.1093/cvr/cvq144

261. Liu H, Yu X, Yu S, Kou J. Molecular mechanisms in lipopolysaccharideinduced pulmonary endothelial barrier dysfunction. Int Immunopharmacol (2015) 29(2):937-46. doi:10.1016/j.intimp.2015.10.010

262. Chen F, Kumar S, Yu Y, Aggarwal S, Gross C, Wang Y, et al. PKC-dependent phosphorylation of eNOS at T495 regulates eNOS coupling and endothelial barrier function in response to G+ -toxins. PLoS One (2014) 9(7):e99823. doi:10.1371/journal.pone.0099823

263. Zemskov E, Lucas R, Verin AD, Umapathy NS. P2Y receptors as regulators of lung endothelial barrier integrity. J Cardiovasc Dis Res (2011) 2(1):14-22. doi:10.4103/0975-3583.78582
264. Jabaudon M, Blondonnet R, Roszyk L, Bouvier D, Audard J, Clairefond G, et al. Soluble receptor for advanced glycation end-products predicts impaired alveolar fluid clearance in acute respiratory distress syndrome. Am J Respir Crit Care Med (2015) 192(2):191-9. doi:10.1164/rccm.201501-0020OC

265. Debruin EJ, Hughes MR, Sina C, Lu A, Cait J, Jian Z, et al. Podocalyxin regulates murine lung vascular permeability by altering endothelial cell adhesion. PLoS One (2014) 9(10):e108881. doi:10.1371/journal.pone.0108881

266. Kostadinova E, Chaput C, Gutbier B, Lippmann J, Sander LE, Mitchell TJ, et al. NLRP3 protects alveolar barrier integrity by an inflammasomeindependent increase of epithelial cell adherence. Sci Rep (2016) 6:30943. doi:10.1038/srep30943

267. Marunaka Y, Tohda H, Hagiwara N, O’Brodovich H. Cytosolic $\mathrm{Ca}(2+)-$ induced modulation of ion selectivity and amiloride sensitivity of a cation channel and beta agonist action in fetal lung epithelium. Biochem Biophys Res Commun (1992) 187(2):648-56. doi:10.1016/0006-291X(92) 91244-K

268. Jain L, Chen XJ, Ramosevac S, Brown LA, Eaton DC. Expression of highly selective sodium channels in alveolar type II cells is determined by culture conditions. Am J Physiol Lung Cell Mol Physiol (2001) 280(4):L646-58.

269. Johnson MD, Bao HF, Helms MN, Chen XJ, Tigue Z, Jain L, et al. Functional ion channels in pulmonary alveolar type I cells support a role for type I cells in lung ion transport. Proc Natl Acad Sci U S A (2006) 103(13):4964-9. doi:10.1073/pnas.0600855103

270. Wang X, Kleyman TR, Tohda H, Marunaka Y, O'Brodovich H. 5(N-Ethyl-N-isopropyl)amiloride sensitive $\mathrm{Na}+$ currents in intact fetal distal lung epithelial cells. Can J Physiol Pharmacol (1993) 71(1):58-62. doi:10.1139/y93-009

271. Chen XJ, Eaton DC, Jain L. Beta-adrenergic regulation of amiloridesensitive lung sodium channels. Am J Physiol Lung Cell Mol Physiol (2002) 282(4):L609-20. doi:10.1152/ajplung.00356.2001

272. Downs CA, Kriener LH, Yu L, Eaton DC, Jain L, Helms MN. beta-adrenergic agonists differentially regulate highly selective and nonselective epithelial sodium channels to promote alveolar fluid clearance in vivo. Am J Physiol Lung Cell Mol Physiol (2012) 302(11):L1167-78. doi:10.1152/ajplung. 00038.2012

273. Jain L, Chen XJ, Brown LA, Eaton DC. Nitric oxide inhibits lung sodium transport through a cGMP-mediated inhibition of epithelial cation channels. Am J Physiol (1998) 274(4 Pt 1):L475-84.

274. Chen XJ, Seth S, Yue G, Kamat P, Compans RW, Guidot D, et al. Influenza virus inhibits $\mathrm{ENaC}$ and lung fluid clearance. Am J Physiol Lung Cell Mol Physiol (2004) 287(2):L366-73. doi:10.1152/ajplung.00011.2004

275. Eaton AF, Yue Q, Eaton DC, Bao HF. ENaC activity and expression is decreased in the lungs of protein kinase C-alpha knockout mice. Am JPhysiol Lung Cell Mol Physiol (2014) 307(5):L374-85. doi:10.1152/ ajplung.00040.2014

276. Ma HP, Li L, Zhou ZH, Eaton DC, Warnock DG. ATP masks stretch activation of epithelial sodium channels in A6 distal nephron cells. Am J Physiol Renal Physiol (2002) 282(3):F501-5. doi:10.1152/ajprenal.00147.2001

277. Davis IC, Matalon S. Epithelial sodium channels in the adult lung important modulators of pulmonary health and disease. Adv Exp Med Biol (2007) 618:127-40. doi:10.1007/978-0-387-75434-5_10

278. Pochynyuk O, Bugaj V, Vandewalle A, Stockand JD. Purinergic control of apical plasma membrane $\mathrm{PI}(4,5) \mathrm{P} 2$ levels sets ENaC activity in principal cells. Am J Physiol Renal Physiol (2008) 294(1):F38-46. doi:10.1152/ajprenal. 00403.2007

279. Stockand JD, Mironova E, Bugaj V, Rieg T, Insel PA, Vallon V, et al. Purinergic inhibition of ENaC produces aldosterone escape. JAm Soc Nephrol (2010) 21(11):1903-11. doi:10.1681/ASN.2010040377

280. Helms MN, Self J, Bao HF, Job LC, Jain L, Eaton DC. Dopamine activates amiloride-sensitive sodium channels in alveolar type I cells in lung slice preparations. Am J Physiol Lung Cell Mol Physiol (2006) 291(4):L610-8. doi:10.1152/ajplung.00426.2005

281. Kapoor N, Lee W, Clark E, Bartoszewski R, McNicholas CM, Latham CB, et al. Interaction of ASIC1 and $\mathrm{ENaC}$ subunits in human glioma cells and rat astrocytes. Am J Physiol Cell Physiol (2011) 300(6):C1246-59. doi:10.1152/ ajpcell.00199.2010

282. Gessner C, Hammerschmidt S, Kuhn H, Seyfarth HJ, Sack U, Engelmann L, et al. Exhaled breath condensate acidification in acute lung injury. Respir Med (2003) 97(11):1188-94. doi:10.1016/S0954-6111(03)00225-7 
283. Suhail $\mathrm{M}$. $\mathrm{Na}(+), \mathrm{K}(+)$-ATPase: ubiquitous multifunctional transmembrane protein and its relevance to various pathophysiological conditions. J Clin Med Res (2010) 2(1):1-17. doi:10.4021/jocmr2010.02.263w

284. Ingbar D, Wendt C, Crandall E. ATPase and the clearance of pulmonary edema fluid. In: Matthay MA, Ingbar DH, editors. Pulmonary Edema. New York: Marcel Dekker (1998). p. 477-99.

285. Brazee PL, Soni PN, Tokhtaeva E, Magnani N, Yemelyanov A, Perlman HR, et al. FXYD5 is an essential mediator of the inflammatory response during lung injury. Front Immunol (2017) 8:623. doi:10.3389/fimmu.2017.00623

286. Kellenberger S, Schild L. Epithelial sodium channel/degenerin family of ion channels: a variety of functions for a shared structure. Physiol Rev (2002) 82(3):735-67. doi:10.1152/physrev.00007.2002

287. McDonough AA, Geering K, Farley RA. The sodium pump needs its beta subunit. FASEB J (1990) 4(6):1598-605.

288. Kaplan JH. Ion movements through the sodium pump. Annu Rev Physiol (1985) 47:535-44. doi:10.1146/annurev.ph.47.030185.002535

289. Kotyk A, Amler E. Na,K-adenosinetriphosphatase: the paradigm of a membrane transport protein. Physiol Res (1995) 44(5):261-74.

290. Geering K. The functional role of beta subunits in oligomeric P-type ATPases. J Bioenerg Biomembr (2001) 33(5):425-38. doi:10.1023/ A:1010623724749

291. Lubarski Gotliv I. FXYD5: $\mathrm{Na}(+) / \mathrm{K}(+)$-ATPase regulator in health and disease. Front Cell Dev Biol (2016) 4:26. doi:10.3389/fcell.2016.00026

292. Scott DW, Walker MP, Sesma J, Wu B, Stuhlmiller TJ, Sabater JR, et al. SPX-101 is a novel epithelial sodium channel-targeted therapeutic for cystic fibrosis that restores mucus transport. Am J Respir Crit Care Med (2017) 196(6):734-44. doi:10.1164/rccm.201612-2445OC

293. Liedtke W. Molecular mechanisms of TRPV4-mediated neural signaling. Ann N Y Acad Sci (2008) 1144:42-52. doi:10.1196/annals.1418.012

294. Scheraga RG, Southern BD, Grove LM, Olman MA. The role of transient receptor potential vanilloid 4 in pulmonary inflammatory diseases. Front Immunol (2017) 8:503. doi:10.3389/fimmu.2017.00503

295. Berridge MJ, Bootman MD, Roderick HL. Calcium signalling: dynamics, homeostasis and remodelling. Nat Rev Mol Cell Biol (2003) 4(7):517-29. doi: $10.1038 / \mathrm{nrm} 1155$

296. Strotmann R, Schultz G, Plant TD. Ca2+-dependent potentiation of the nonselective cation channel TRPV4 is mediated by a C-terminal calmodulin binding site. J Biol Chem (2003) 278(29):26541-9. doi:10.1074/jbc. M302590200

297. Zhu MX. Multiple roles of calmodulin and other $\mathrm{Ca}(2+)$-binding proteins in the functional regulation of TRP channels. Pflugers Arch (2005) 451(1): 105-15. doi:10.1007/s00424-005-1427-1

298. White DB. 104 - end-of-life care in respiratory failure A2 - Broaddus, V. Courtney. 6th ed. In: Mason RJ, Ernst JD, King TE, Lazarus SC, Murray JF, Nadel JA, Slutsky AS, Gotway MB, editors. Murray and Nadel's Textbook of Respiratory Medicine. Philadelphia: W.B. Saunders (2016). p. 1807-20.

299. Yang XR, Lin MJ, McIntosh LS, Sham JS. Functional expression of transient receptor potential melastatin- and vanilloid-related channels in pulmonary arterial and aortic smooth muscle. Am JPhysiol Lung Cell Mol Physiol (2006) 290(6):L1267-76. doi:10.1152/ajplung.00515.2005

300. Moran MM, McAlexander MA, Biro T, Szallasi A. Transient receptor potential channels as therapeutic targets. Nat Rev Drug Discov (2011) 10(8): 601-20. doi:10.1038/nrd3456

301. Yang XR, Lin AH, Hughes JM, Flavahan NA, Cao YN, Liedtke W, et al. Upregulation of osmo-mechanosensitive TRPV4 channel facilitates chronic hypoxia-induced myogenic tone and pulmonary hypertension. Am J Physiol Lung Cell Mol Physiol (2012) 302(6):L555-68. doi:10.1152/ajplung. 00005.2011

302. Suresh K, Servinsky L, Reyes J, Baksh S, Undem C, Caterina M, et al. Hydrogen peroxide-induced calcium influx in lung microvascular endothelial cells involves TRPV4. Am J Physiol Lung Cell Mol Physiol (2015) 309(12): L1467. doi:10.1152/ajplung.00275.2015

303. Parpaite T, Cardouat G, Mauroux M, Gillibert-Duplantier J, Robillard P, Quignard JF, et al. Effect of hypoxia on TRPV1 and TRPV4 channels in rat pulmonary arterial smooth muscle cells. Pflugers Arch (2016) 468(1):111-30. doi:10.1007/s00424-015-1704-6

304. Borish LC, Nelson HS, Corren J, Bensch G, Whitmore JB, Busse WW. Efficacy of soluble IL-4 receptor for the treatment of adults with asthma. J Allergy Clin Immunol (2001) 107(6):963-70. doi:10.1067/mai.2001.115624
305. Hamanaka K, Jian MY, Weber DS, Alvarez DF, Townsley MI, Al-Mehdi AB, et al. TRPV4 initiates the acute calcium-dependent permeability increase during ventilator-induced lung injury in isolated mouse lungs. Am J Physiol Lung Cell Mol Physiol (2007) 293(4):L923-32. doi:10.1152/ajplung. 00221.2007

306. Zhou G, Dada LA, Wu M, Kelly A, Trejo H, Zhou Q, et al. Hypoxia-induced alveolar epithelial-mesenchymal transition requires mitochondrial ROS and hypoxia-inducible factor 1. Am J Physiol Lung Cell Mol Physiol (2009) 297(6):L1120-30. doi:10.1152/ajplung.00007.2009

307. Balakrishna S, Song W, Achanta S, Doran SF, Liu B, Kaelberer MM, et al. TRPV4 inhibition counteracts edema and inflammation and improves pulmonary function and oxygen saturation in chemically induced acute lung injury. Am J Physiol Lung Cell Mol Physiol (2014) 307(2):L158-72. doi:10.1152/ajplung.00065.2014

308. Rahaman SO, Grove LM, Paruchuri S, Southern BD, Abraham S, Niese KA, et al. TRPV4 mediates myofibroblast differentiation and pulmonary fibrosis in mice. J Clin Invest (2014) 124(12):5225-38. doi:10.1172/jci75331

309. Henry CO, Dalloneau E, Perez-Berezo MT, Plata C, Wu Y, Guillon A, et al. In vitro and in vivo evidence for an inflammatory role of the calcium channel TRPV4 in lung epithelium: potential involvement in cystic fibrosis. Am J Physiol Lung Cell Mol Physiol (2016) 311(3):L664-75. doi:10.1152/ ajplung.00442.2015

310. Scheraga RG, Abraham S, Niese KA, Southern BD, Grove LM, Hite RD, et al. TRPV4 mechanosensitive ion channel regulates lipopolysaccharidestimulated macrophage phagocytosis. JImmunol (2016) 196(1):428-36. doi:10.4049/jimmunol.1501688

311. Cioffi DL, Lowe K, Alvarez DF, Barry C, Stevens T. TRPing on the lung endothelium: calcium channels that regulate barrier function. Antioxid Redox Signal (2009) 11(4):765-76. doi:10.1089/ars.2008.2221

312. Jia Y, Wang X, Varty L, Rizzo CA, Yang R, Correll CC, et al. Functional TRPV4 channels are expressed in human airway smooth muscle cells. Am J Physiol Lung Cell Mol Physiol (2004) 287(2):L272-8. doi:10.1152/ajplung.00393.2003

313. Dalsgaard T, Sonkusare SK, Teuscher C, Poynter ME, Nelson MT. Pharmacological inhibitors of TRPV4 channels reduce cytokine production, restore endothelial function and increase survival in septic mice. Sci Rep (2016) 6:33841. doi:10.1038/srep33841

314. Yin J, Michalick L, Tang C, Tabuchi A, Goldenberg N, Dan Q, et al. Role of transient receptor potential vanilloid 4 in neutrophil activation and acute lung injury. Am J Respir Cell Mol Biol (2016) 54(3):370-83. doi:10.1165/ rcmb.2014-0225OC

315. Zhu G, Gulsvik A, Bakke P, Ghatta S, Anderson W, Lomas DA, et al. Association of TRPV4 gene polymorphisms with chronic obstructive pulmonary disease. Hum Mol Genet (2009) 18(11):2053-62. doi:10.1093/hmg/ddp111

316. McAlexander MA, Luttmann MA, Hunsberger GE, Undem BJ. Transient receptor potential vanilloid 4 activation constricts the human bronchus via the release of cysteinyl leukotrienes. J Pharmacol Exp Ther (2014) 349(1): 118-25. doi:10.1124/jpet.113.210203

317. Satir P, Sleigh MA. The physiology of cilia and mucociliary interactions. Annu Rev Physiol (1990) 52:137-55. doi:10.1146/annurev.ph.52.030190.001033

318. Salathe M. Regulation of mammalian ciliary beating. Annu Rev Physiol (2007) 69:401-22. doi:10.1146/annurev.physiol.69.040705.141253

319. Jurek SC, Hirano-Kobayashi M, Chiang H, Kohane DS, Matthews BD. Prevention of ventilator-induced lung edema by inhalation of nanoparticles releasing ruthenium red. Am J Respir Cell Mol Biol (2014) 50(6):1107-17. doi:10.1165/rcmb.2013-0163OC

320. Wynn TA, Chawla A, Pollard JW. Macrophage biology in development, homeostasis and disease. Nature (2013) 496(7446):445-55. doi:10.1038/ nature12034

321. Jian MY, King JA, Al-Mehdi AB, Liedtke W, Townsley MI. High vascular pressure-induced lung injury requires $\mathrm{P} 450$ epoxygenase-dependent activation of TRPV4. Am J Respir Cell Mol Biol (2008) 38(4):386-92. doi:10.1165/ rcmb.2007-0192OC

322. Wu S, Jian MY, Xu YC, Zhou C, Al-Mehdi AB, Liedtke W, et al. Ca2+ entry via alphalG and TRPV4 channels differentially regulates surface expression of P-selectin and barrier integrity in pulmonary capillary endothelium. Am J Physiol Lung Cell Mol Physiol (2009) 297(4):L650-7. doi:10.1152/ ajplung.00015.2009

323. Hamanaka K, Jian MY, Townsley MI, King JA, Liedtke W, Weber DS, et al. TRPV4 channels augment macrophage activation and ventilator-induced 
lung injury. Am J Physiol Lung Cell Mol Physiol (2010) 299(3):L353-62. doi:10.1152/ajplung.00315.2009

324. Van Goethem E, Poincloux R, Gauffre F, Maridonneau-Parini I, Le Cabec V. Matrix architecture dictates three-dimensional migration modes of human macrophages: differential involvement of proteases and podosome-like structures. J Immunol (2010) 184(2):1049-61. doi:10.4049/jimmunol. 0902223

325. Blakney AK, Swartzlander MD, Bryant SJ. The effects of substrate stiffness on the in vitro activation of macrophages and in vivo host response to poly(ethylene glycol)-based hydrogels. J Biomed Mater Res A (2012) 100(6): 1375-86. doi:10.1002/jbm.a.34104

326. Murray JF, Nadel JA. Preface to the sixth edition. In: Murray JF, Nadel JA, Broaddus VC, Mason RJ, Ernst JD, King TE Jr., editors. Murray and Nadel's Textbook of Respiratory Medicine. 6th ed. Philadelphia: Elsevier Saunders (2016). p. 1-3.

327. Akei H, Whitsett JA, Buroker M, Ninomiya T, Tatsumi H, Weaver TE, et al. Surface tension influences cell shape and phagocytosis in alveolar macrophages. Am J Physiol Lung Cell Mol Physiol (2006) 291(4):L572-9. doi:10.1152/ajplung.00060.2006

328. Malczyk M, Erb A, Veith C, Ghofrani HA, Schermuly RT, Gudermann T, et al. The role of transient receptor potential channel 6 channels in the pulmonary vasculature. Front Immunol (2017) 8:707. doi:10.3389/fimmu. 2017.00707

329. Sanjabi S, Zenewicz LA, Kamanaka M, Flavell RA. Anti-inflammatory and pro-inflammatory roles of TGF-beta, IL-10, and IL-22 in immunity and autoimmunity. Curr Opin Pharmacol (2009) 9(4):447-53. doi:10.1016/j. coph.2009.04.008

330. Kelley J. Cytokines of the lung. Am Rev Respir Dis (1990) 141(3):765-88. doi:10.1164/ajrccm/141.3.765

331. Fiers W. Tumor necrosis factor characterization at the molecular, cellular and in vivo level. FEBS Lett (1991) 285(2):199-212. doi:10.1016/00145793(91)80803-B

332. Szatmary Z. Tumor necrosis factor-alpha: molecular-biological aspects minireview. Neoplasma (1999) 46(5):257-66.

333. Hocking DC, Ferro TJ, Johnson A. Dextran sulfate inhibits PMN-dependent hydrostatic pulmonary edema induced by tumor necrosis factor. J Appl Physiol (1985) (1991) 70(3):1121-8.

334. Lo SK, Everitt J, Gu J, Malik AB. Tumor necrosis factor mediates experimental pulmonary edema by ICAM-1 and CD18-dependent mechanisms. J Clin Invest (1992) 89(3):981-8. doi:10.1172/jci115681

335. Horgan MJ, Palace GP, Everitt JE, Malik AB. TNF-alpha release in endotoxemia contributes to neutrophil-dependent pulmonary edema. Am J Physiol (1993) 264(4 Pt 2):H1161-5.

336. Faggioni R, Gatti S, Demitri MT, Delgado R, Echtenacher B, Gnocchi P, et al. Role of xanthine oxidase and reactive oxygen intermediates in LPSand TNF-induced pulmonary edema. J Lab Clin Med (1994) 123(3):394-9.

337. Lo SK, Bevilacqua B, Malik AB. E-selectin ligands mediate tumor necrosis factor-induced neutrophil sequestration and pulmonary edema in guinea pig lungs. Circ Res (1994) 75(6):955-60. doi:10.1161/01.RES.75.6.955

338. Koh Y, Hybertson BM, Jepson EK, Repine JE. Tumor necrosis factor induced acute lung leak in rats: less than with interleukin-1. Inflammation (1996) 20:461. doi:10.1007/BF01487039

339. Wallach D, Varfolomeev EE, Malinin NL, Goltsev YV, Kovalenko AV, Boldin MP. Tumor necrosis factor receptor and Fas signaling mechanisms. Annu Rev Immunol (1999) 17:331-67. doi:10.1146/annurev.immunol.17.1.331

340. Wallach D. The cybernetics of TNF: old views and newer ones. Semin Cell Dev Biol (2016) 50:105-14. doi:10.1016/j.semcdb.2015.10.014

341. Mannel DN, Echtenacher B. TNF in the inflammatory response. Chem Immunol (2000) 74:141-61. doi:10.1159/000058757

342. Locksley RM, Killeen N, Lenardo MJ. The TNF and TNF receptor superfamilies: integrating mammalian biology. Cell (2001) 104(4):487-501. doi:10.1016/S0092-8674(01)00237-9

343. Keane J. TNF-blocking agents and tuberculosis: new drugs illuminate an old topic. Rheumatology (Oxford) (2005) 44(6):714-20. doi:10.1093/ rheumatology/keh567

344. Hundsberger H, Verin A, Wiesner C, Pfluger M, Dulebo A, Schutt W, et al. TNF: a moonlighting protein at the interface between cancer and infection. Front Biosci (2008) 13:5374-86. doi:10.2741/3087
345. Aggarwal BB. Signalling pathways of the TNF superfamily: a double-edged sword. Nat Rev Immunol (2003) 3(9):745-56. doi:10.1038/nril184

346. Harris J, Keane J. How tumour necrosis factor blockers interfere with tuberculosis immunity. Clin Exp Immunol (2010) 161(1):1-9. doi:10.1111/ j.1365-2249.2010.04146.x

347. Berry MA, Hargadon B, Shelley M, Parker D, Shaw DE, Green RH, et al. Evidence of a role of tumor necrosis factor alpha in refractory asthma. $N$ Engl J Med (2006) 354(7):697-708. doi:10.1056/NEJMoa050580

348. Mukhopadhyay S, Hoidal JR, Mukherjee TK. Role of TNFo in pulmonary pathophysiology. Respir Res (2006) 7(1):125. doi:10.1186/1465-9921-7-125

349. Balkwill F. Tumour necrosis factor and cancer. Nat Rev Cancer (2009) 9(5):361-71. doi:10.1038/nrc2628

350. Zhang H, Park Y, Wu J, Chen X, Lee S, Yang J, et al. Role of TNF-alpha in vascular dysfunction. Clin Sci (Lond) (2009) 116(3):219-30. doi:10.1042/ cs20080196

351. Hession C, Decker JM, Sherblom AP, Kumar S, Yue CC, Mattaliano RJ, et al. Uromodulin (Tamm-Horsfall glycoprotein): a renal ligand for lymphokines. Science (1987) 237(4821):1479-84. doi:10.1126/science.3498215

352. Sherblom AP, Decker JM, Muchmore AV. The lectin-like interaction between recombinant tumor necrosis factor and uromodulin. J Biol Chem (1988) 263(11):5418-24.

353. Lucas R, Magez S, De Leys R, Fransen L, Scheerlinck J-P, Rambelberg M, et al. Mapping the lectin-like activity of tumor necrosis factor. Science (1994) 263(5148):814-8. doi:10.1126/science.8303299

354. Fronius M. Treatment of pulmonary edema by ENaC activators/stimulators. Curr Mol Pharmacol (2013) 6(1):13-27. doi:10.2174/1874467211306010003

355. Hartmann EK, Boehme S, Duenges B, Bentley A, Klein KU, Kwiecien R, et al. An inhaled tumor necrosis factor-alpha-derived TIP peptide improves the pulmonary function in experimental lung injury. Acta Anaesthesiol Scand (2013) 57(3):334-41. doi:10.1111/aas.12034

356. Schwameis R, Eder S, Pietschmann H, Fischer B, Mascher H, Tzotzos S, et al. A FIM study to assess safety and exposure of inhaled single doses of AP301-A specific $\mathrm{ENaC}$ channel activator for the treatment of acute lung injury. J Clin Pharmacol (2014) 54(3):341-50. doi:10.1002/jcph.203

357. Aigner C, Slama A, Barta M, Mitterbauer A, Lang G, Taghavi S, et al. Treatment of primary graft dysfunction after lung transplantation with orally inhaled AP301: a prospective, randomized pilot study. J Heart Lung Transplant (2017) pii: S1053-2498(17):32036-32033. doi:10.1016/j.healun.2017.09.021

358. Krenn K, Lucas R, Croize A, Boehme S, Klein KU, Hermann R, et al. Inhaled AP301 for treatment of pulmonary edema in mechanically ventilated patients with acute respiratory distress syndrome: a phase IIa randomized placebo-controlled trial. Crit Care (2017) 21(1):194. doi:10.1186/s13054017-1795-x

359. Yamagata T, Yamagata Y, Nishimoto T, Hirano T, Nakanishi M, Minakata Y, et al. The regulation of amiloride-sensitive epithelial sodium channels by tumor necrosis factor-alpha in injured lungs and alveolar type II cells. Respir Physiol Neurobiol (2009) 166(1):16-23. doi:10.1016/j.resp.2008.12.008

360. Lucas R, Czikora I, Sridhar S, Zemskov E, Gorshkov B, Siddaramappa U, et al. Mini-review: novel therapeutic strategies to blunt actions of pneumolysin in the lungs. Toxins (2013) 5(7):1244-60. doi:10.3390/toxins5071244

361. Wilson MR, Wakabayashi K, Bertok S, Oakley CM, Patel BV, O’Dea KP, et al. Inhibition of TNF receptor p55 by a domain antibody attenuates the initial phase of acid-induced lung injury in mice. Front Immunol (2017) 8:128. doi:10.3389/fimmu.2017.00128

362. Wilson MR, Goddard ME, O'Dea KP, Choudhury S, Takata M. Differential roles of p55 and p75 tumor necrosis factor receptors on stretch-induced pulmonary edema in mice. Am J Physiol Lung Cell Mol Physiol (2007) 293(1):L60-8. doi:10.1152/ajplung.00284.2006

363. Wright TW, Pryhuber GS, Chess PR, Wang Z, Notter RH, Gigliotti F. TNF receptor signaling contributes to chemokine secretion, inflammation, and respiratory deficits during pneumocystis pneumonia. JImmunol (2004) 172(4):2511-21. doi:10.4049/jimmunol.172.4.2511

364. Guo RF, Ward PA. Mediators and regulation of neutrophil accumulation in inflammatory responses in lung: insights from the $\mathrm{IgG}$ immune complex model. Free Radic Biol Med (2002) 33(3):303-10. doi:10.1016/S08915849(02)00823-7

365. Rezaiguia S, Garat C, Delclaux C, Meignan M, Fleury J, Legrand P, et al. Acute bacterial pneumonia in rats increases alveolar epithelial fluid 
clearancebyatumor necrosisfactor-alpha-dependent mechanism.J Clin Invest (1997) 99(2):325-35. doi:10.1172/JCI119161

366. Borjesson A, Norlin A, Wang X, Andersson R, Folkesson HG. TNF-a stimulates alveolar liquid clearance during intestinal ischemia-reperfusion in rats. Am J Physiol Lung Cell Mol Physiol (2000) 278:L3-12.

367. Vilcek J, Feldmann M. Historical review: cytokines as therapeutics and targets of therapeutics. Trends Pharmacol Sci (2004) 25(4):201-9. doi:10.1016/ j.tips.2004.02.011

368. Yi ES, Ulich TR. Endotoxin, interleukin-1, and tumor necrosis factor cause neutrophil-dependent microvascular leakage in postcapillary venules. Am J Pathol (1992) 140(3):659-63.

369. Hammond ME, Lapointe GR, Feucht PH, Hilt S, Gallegos CA, Gordon CA, et al. IL-8 induces neutrophil chemotaxis predominantly via type I IL-8 receptors. J Immunol (1995) 155(3):1428-33.

370. Narasaraju T, Yang E, Samy RP, Ng HH, Poh WP, Liew AA, et al. Excessive neutrophils and neutrophil extracellular traps contribute to acute lung injury of influenza pneumonitis. Am J Pathol (2011) 179(1):199-210. doi:10.1016/j.ajpath.2011.03.013

371. Peteranderl C, Sznajder JI, Herold S, Lecuona E. Inflammatory responses regulating alveolar ion transport during pulmonary infections. Front Immunol (2017) 8:446. doi:10.3389/fimmu.2017.00446

372. Petrache I, Verin AD, Crow MT, Birukova A, Liu F, Garcia JG. Differential effect of MLC kinase in TNF-alpha-induced endothelial cell apoptosis and barrier dysfunction. Am JPhysiol Lung Cell Mol Physiol (2001) 280(6):L1168-78.

373. Goldblum SE, Hennig B, Jay M, Yoneda K, McClain CJ. Tumor necrosis factor alpha-induced pulmonary vascular endothelial injury. Infect Immun (1989) 57(4):1218-26.

374. Brett J, Gerlach H, Nawroth P, Steinberg S, Godman G, Stern D. Tumor necrosis factor/cachectin increases permeability of endothelial cell monolayers by a mechanism involving regulatory G proteins. J Exp Med (1989) 169(6):1977-91. doi:10.1084/jem.169.6.1977

375. Wheatley EM, Vincent PA, McKeown-Longo PJ, Saba TM. Effect of fibronectin on permeability of normal and TNF-treated lung endothelial cell monolayers. Am J Physiol (1993) 264(1 Pt 2):R90-6.

376. Partridge CA, Horvath CJ, Del Vecchio PJ, Phillips PG, Malik AB. Influence of extracellular matrix in tumor necrosis factor-induced increase in endothelial permeability. Am J Physiol (1992) 263(6 Pt 1):L627-33.

377. Jahr J, Grande PO. In vivo effects of tumor necrosis factor-alpha on capillary permeability and vascular tone in a skeletal muscle. Acta Anaesthesiol Scand (1996) 40(2):256-61. doi:10.1111/j.1399-6576.1996.tb04429.x

378. Lum H, Roebuck KA. Oxidant stress and endothelial cell dysfunction. Am J Physiol Cell Physiol (2001) 280(4):C719-41.

379. Hamvas A, Palazzo R, Kaiser L, Cooper J, Shuman T, Velazquez M, et al. Inflammation and oxygen free radical formation during pulmonary ischemia-reperfusion injury. J Appl Physiol (1985) (1992) 72(2):621-8.

380. Ischiropoulos $\mathrm{H}$, al-Mehdi $\mathrm{AB}$, Fisher $\mathrm{AB}$. Reactive species in ischemic rat lung injury: contribution of peroxynitrite. Am J Physiol (1995) 269(2 Pt 1): L158-64.

381. Peralta C, Bulbena O, Xaus C, Prats N, Cutrin JC, Poli G, et al. Ischemic preconditioning: a defense mechanism against the reactive oxygen species generated after hepatic ischemia reperfusion. Transplantation (2002) 73(8): 1203-11. doi:10.1097/00007890-200204270-00004

382. Guo Y, DuVall MD, Crow JP, Matalon S. Nitric oxide inhibits Na+ absorption across cultured alveolar type II monolayers. Am J Physiol (1998) 274(3 Pt 1): L369-77.

383. Peschon JJ, Slack JL, Reddy P, Stocking KL, Sunnarborg SW, Lee DC, et al. An essential role for ectodomain shedding in mammalian development. Science (1998) 282(5392):1281-4. doi:10.1126/science.282.5392.1281

384. Luks AM, Schoene RB, Swenson ER. High altitude. In: Broaddus VC, Mason RJ, Ernst JD, King TE, Lazarus SC, Murray JF, Slutsky AS, Gotway MB, editors. Murray \& Nadel's Texpbook of Respiratory Medicine. Philadelphia, USA: Elsevier (2016). p. 1367-84.

385. Bartsch P, Mairbaurl H, Maggiorini M, Swenson ER. Physiological aspects of high-altitude pulmonary edema. J Appl Physiol (1985) (2005) 98(3): 1101-10. doi:10.1152/japplphysiol.01167.2004

386. Leshem E, Pandey P, Shlim DR, Hiramatsu K, Sidi Y, Schwartz E. Clinical features of patients with severe altitude illness in Nepal. J Travel Med (2008) 15(5):315-22. doi:10.1111/j.1708-8305.2008.00229.x
387. Sartori C, Matthay MA. Alveolar epithelial fluid transport in acute lung injury: new insights. Eur Respir J (2002) 20(5):1299-313. doi:10.1183/090 31936.02.00401602

388. Alli AA, Bao HF, Alli AA, Aldrugh Y, Song JZ, Ma HP, et al. Phosphatidylinositol phosphate-dependent regulation of Xenopus ENaC by MARCKS protein. Am J Physiol Renal Physiol (2012) 303(6):F800-11. doi:10.1152/ ajprenal.00703.2011

389. Collier DM, Tomkovicz VR, Peterson ZJ, Benson CJ, Snyder PM. Intersubunit conformational changes mediate epithelial sodium channel gating. J Gen Physiol (2014) 144(4):337-48. doi:10.1085/jgp.201411208

390. Wiley SR, Schooley K, Smolak PJ, Din WS, Huang C-P, Nicholl JK, et al. Identification and characterization of a new member of the TNF family that induces apoptosis. Immunity (1995) 3(6):673-82. doi:10.1016/10747613(95) $90057-8$

391. Fensterl V, Sen GC. Interferons and viral infections. Biofactors (2009) 35(1):14-20. doi:10.1002/biof.6

392. Brincks EL, Katewa A, Kucaba TA, Griffith TS, Legge KL. CD8 T cells utilize TRAIL to control influenza virus infection. J Immunol (2008) 181(10):7428. doi:10.4049/jimmunol.181.10.7428-a

393. Herold S, Steinmueller M, von Wulffen W, Cakarova L, Pinto R, Pleschka S, et al. Lung epithelial apoptosis in influenza virus pneumonia: the role of macrophage-expressed TNF-related apoptosis-inducing ligand. J Exp Med (2008) 205(13):3065-77. doi:10.1084/jem.20080201

394. Ellis GT, Davidson S, Crotta S, Branzk N, Papayannopoulos V, Wack A. TRAIL+ monocytes and monocyte-related cells cause lung damage and thereby increase susceptibility to influenza-Streptococcus pneumoniae coinfection. EMBO Rep (2015) 16(9):1203-18. doi:10.15252/embr.201540473

395. Matthay MA, Ware LB, Zimmerman GA. The acute respiratory distress syndrome. J Clin Invest (2012) 122(8):2731-40. doi:10.1172/jci60331

396. Gonzales JN, Lucas R, Verin AD. The acute respiratory distress syndrome: mechanisms and perspective therapeutic approaches. Austin J Vasc Med (2015) 2(1):ii:1009.

397. Millan FA, Denhez F, Kondaiah P, Akhurst RJ. Embryonic gene expression patterns of TGF beta 1, beta 2 and beta 3 suggest different developmental functions in vivo. Development (1991) 111(1):131-43.

398. Worthington JJ, Fenton TM, Czajkowska BI, Klementowicz JE, Travis MA Regulation of TGF $\beta$ in the immune system: an emerging role for integrins and dendritic cells. Immunobiology (2012) 217(12):1259-65. doi:10.1016/ j.imbio.2012.06.009

399. Kumar V, Abbas A, Aster J. Robbins Basic Pathology. Tenth ed. Pennsylvania: Elsevier (2017).

400. Branton MH, Kopp JB. TGF-beta and fibrosis. Microbes Infect (1999) 1(15):1349-65. doi:10.1016/S1286-4579(99)00250-6

401. Massague J, Andres J, Attisano L, Cheifetz S, Lopez-Casillas F, Ohtsuki M, et al. TGF-beta receptors. Mol Reprod Dev (1992) 32(2):99-104. doi:10.1002/ mrd.1080320204

402. Akhurst RJ, Hata A. Targeting the TGF $\beta$ signalling pathway in disease. Nat Rev Drug Discov (2012) 11(10):790-811. doi:10.1038/nrd3810

403. Yang H, Cao C, Wu C, Yuan C, Gu Q, Shi Q, et al. TGF- $\beta$ l suppresses inflammation in cell therapy for intervertebral disc degeneration. Sci Rep (2015) 5:13254. doi:10.1038/srep13254

404. Pohlers D, Brenmoehl J, Löffler I, Müller CK, Leipner C, Schultze-Mosgau S, et al. TGF- $\beta$ and fibrosis in different organs - molecular pathway imprints. Biochim Biophys Acta (2009) 1792(8):746-56. doi:10.1016/j.bbadis.2009. 06.004

405. Letterio JJ, Roberts AB. Regulation of immune responses by TGF-beta. Annu Rev Immunol (1998) 16:137-61. doi:10.1146/annurev.immunol.16.1.137

406. Filippi CM, Juedes AE, Oldham JE, Ling E, Togher L, Peng Y, et al. Transforming growth factor-beta suppresses the activation of CD8+ T-cells when naive but promotes their survival and function once antigen experienced: a two-faced impact on autoimmunity. Diabetes (2008) 57(10):2684-92. doi:10.2337/db08-0609

407. Guidot DM, Modelska K, Lois M, Jain L, Moss IM, Pittet J-F, et al. Ethanol ingestion via glutathione depletion impairs alveolar epithelial barrier function in rats. Am J Physiol Lung Cell Mol Physiol (2000) 279(1):L127.

408. Annes JP, Munger JS, Rifkin DB. Making sense of latent TGF $\beta$ activation. J Cell Sci (2003) 116(2):217-24. doi:10.1242/jcs.00229

409. Munger JS, Huang X, Kawakatsu H, Griffiths MJD, Dalton SL, Wu J, et al. A mechanism for regulating pulmonary inflammation and fibrosis: the 
integrin $\alpha v \beta 6$ binds and activates latent TGF $\beta 1$. Cell (1999) 96(3):319-28. doi:10.1016/S0092-8674(00)80545-0

410. Bickel M. The role of interleukin-8 in inflammation and mechanisms of regulation. J Periodontol (1993) 64(5 Suppl):456-60.

411. Chen Z, Shao X, Dou X, Zhang X, Wang Y, Zhu C, et al. Role of the Mycoplasma pneumoniae/interleukin-8/neutrophil axis in the pathogenesis of pneumonia. PLoS One (2016) 11(1):e0146377. doi:10.1371/journal.pone.0146377

412. Goodman RB, Strieter RM, Martin DP, Steinberg KP, Milberg JA, Maunder RJ, et al. Inflammatory cytokines in patients with persistence of the acute respiratory distress syndrome. Am J Respir Crit Care Med (1996) 154(3 Pt 1):602-11. doi:10.1164/ajrccm.154.3.8810593

413. Kurdowska A, Miller EJ, Noble JM, Baughman RP, Matthay MA, Brelsford WG, et al. Anti-IL-8 autoantibodies in alveolar fluid from patients with the adult respiratory distress syndrome. J Immunol (1996) 157(6):2699-706.

414. Pease JE, Sabroe I. The role of interleukin- 8 and its receptors in inflammatory lung disease. Am J Respir Med (2002) 1(1):19-25. doi:10.1007/BF03257159

415. Miller EJ, Cohen AB, Matthay MA. Increased interleukin-8 concentrations in the pulmonary edema fluid of patients with acute respiratory distress syndrome from sepsis. Crit Care Med (1996) 24(9):1448-54. doi:10.1097/00003246-199609000-00004

416. Wagener BM, Roux J, Carles M, Pittet JF. Synergistic inhibition of beta2adrenergic receptor-mediated alveolar epithelial fluid transport by interleukin-8 and transforming growth factor-beta. Anesthesiology (2015) 122(5):1084-92. doi:10.1097/ALN.0000000000000595

417. Meduri GU, Headley S, Kohler G, Stentz F, Tolley E, Umberger R, et al. Persistent elevation of inflammatory cytokines predicts a poor outcome in ARDS: plasma IL-1 $\beta$ and IL-6 levels are consistent and efficient predictors of outcome over time. Chest (1995) 107(4):1062-73. doi:10.1378/ chest.107.4.1062

418. Matute-Bello G, Frevert CW, Liles WC, Nakamura M, Ruzinski JT, Ballman K, et al. Fas/Fas ligand system mediates epithelial injury, but not pulmonary host defenses, in response to inhaled bacteria. Infect Immun (2001) 69(9):5768-76. doi:10.1128/IAI.69.9.5768-5776.2001

419. Bem RA, Farnand AW, Wong V, Koski A, Rosenfeld ME, van Rooijen N, et al. Depletion of resident alveolar macrophages does not prevent Fasmediated lung injury in mice. Am J Physiol Lung Cell Mol Physiol (2008) 295(2):L314-25. doi:10.1152/ajplung.00210.2007

420. Farnand AW, Eastman AJ, Herrero R, Hanson JF, Mongovin S, Altemeier WA, et al. Fas activation in alveolar epithelial cells induces KC (CXCL1) release by a MyD88-dependent mechanism. Am J Respir Cell Mol Biol (2011) 45(3):650-8. doi:10.1165/rcmb.2010-0153OC

421. Ye X, Acharya R, Herbert JB, Hamilton SE, Folkesson HG. IL-1beta stimulates alveolar fluid absorption in fetal guinea pig lungs via the hypothalamus-pituitary-adrenal gland axis. Am J Physiol Lung Cell Mol Physiol (2004) 286(4):L756-66. doi:10.1152/ajplung.00214.2003

422. Mason CM, Guery BP, Summer WR, Nelson S. Keratinocyte growth factor attenuates lung leak induced by alpha-naphthylthiourea in rats. Crit Care Med (1996) 24(6):925-31. doi:10.1097/00003246-199606000-00009

423. Guery BP, Mason CM, Dobard EP, Beaucaire G, Summer WR, Nelson S. Keratinocyte growth factor increases transalveolar sodium reabsorption in normal and injured rat lungs. Am J Respir Crit Care Med (1997) 155(5): 1777-84. doi:10.1164/ajrccm.155.5.9154891

424. Borok Z, Danto SI, Dimen LL, Zhang XL, Lubman RL. Na(+)-K(+)ATPase expression in alveolar epithelial cells: upregulation of active ion transport by KGF. Am J Physiol (1998) 274(1 Pt 1):L149-58.

425. Verghese GM, McCormick-Shannon K, Mason RJ, Matthay MA. Hepatocyte growth factor and keratinocyte growth factor in the pulmonary edema fluid of patients with acute lung injury. Biologic and clinical significance. Am J Respir Crit Care Med (1998) 158(2):386-94. doi:10.1164/ajrccm.158. 2.9711111

426. Yi ES, Salgado M, Williams S, Kim SJ, Masliah E, Yin S, et al. Keratinocyte growth factor decreases pulmonary edema, transforming growth factor-beta and platelet-derived growth factor-BB expression, and alveolar type II cell loss in bleomycin-induced lung injury. Inflammation (1998) 22(3):315-25. doi:10.1023/A:1022304317111

427. Wang Y, Folkesson HG, Jayr C, Ware LB, Matthay MA. Alveolar epithelial fluid transport can be simultaneously upregulated by both KGF and betaagonist therapy. J Appl Physiol (1985) (1999) 87(5):1852-60.
428. Viget NB, Guery BP, Ader F, Neviere R, Alfandari S, Creuzy C, et al. Keratinocyte growth factor protects against Pseudomonas aeruginosa-induced lung injury. Am J Physiol Lung Cell Mol Physiol (2000) 279(6):L1199-209.

429. Welsh DA, Summer WR, Dobard EP, Nelson S, Mason CM. Keratinocyte growth factor prevents ventilator-induced lung injury in an ex vivo rat model. Am J Respir Crit Care Med (2000) 162(3 Pt 1):1081-6. doi:10.1164/ ajrccm.162.3.9908099

430. Welsh DA, Guery BP, Deboisblanc BP, Dobard EP, Creusy C, Mercante D, et al. Keratinocyte growth factor attenuates hydrostatic pulmonary edema in an isolated, perfused rat lung model. Am J Physiol Heart Circ Physiol (2001) 280(3):H1311-7.

431. Goolaerts A, Pellan-Randrianarison N, Larghero J, Vanneaux V, Uzunhan Y, Gille $\mathrm{T}$, et al. Conditioned media from mesenchymal stromal cells restore sodium transport and preserve epithelial permeability in an in vitro model of acute alveolar injury. Am J Physiol Lung Cell Mol Physiol (2014) 306(11):975-85. doi:10.1152/ajplung.00242.2013

432. McAuley DF, Cross LM, Hamid U, Gardner E, Elborn JS, Cullen KM, et al. Keratinocyte growth factor for the treatment of the acute respiratory distress syndrome (KARE): a randomised, double-blind, placebocontrolled phase 2 trial. Lancet Respir Med (2017) 5(6):484-91. doi:10.1016/ S2213-2600(17)30171-6

433. Neeper M, Schmidt AM, Brett J, Yan SD, Wang F, Pan YC, et al. Cloning and expression of a cell surface receptor for advanced glycosylation end products of proteins. J Biol Chem (1992) 267(21):14998-5004.

434. Fehrenbach H, Kasper M, Tschernig T, Shearman MS, Schuh D, Muller M. Receptor for advanced glycation endproducts (RAGE) exhibits highly differential cellular and subcellular localisation in rat and human lung. Cell Mol Biol (Noisy-le-grand) (1998) 44(7):1147-57.

435. Shirasawa M, Fujiwara N, Hirabayashi S, Ohno H, Iida J, Makita K, et al. Receptor for advanced glycation end-products is a marker of type I lung alveolar cells. Genes Cells (2004) 9(2):165-74. doi:10.1111/j.1356-9597.2004. 00712.x

436. Vazzana N, Santilli F, Cuccurullo C, Davi G. Soluble forms of RAGE in internal medicine. Intern Emerg Med (2009) 4(5):389-401. doi:10.1007/ s11739-009-0300-1

437. Sparvero LJ, Asafu-Adjei D, Kang R, Tang D, Amin N, Im J, et al. RAGE (receptor for advanced glycation endproducts), RAGE ligands, and their role in cancer and inflammation. J Transl Med (2009) 7:17-17. doi:10.1186/1479-5876-7-17

438. Lee EJ, Park JH. Receptor for advanced glycation endproducts (RAGE), its ligands, and soluble RAGE: potential biomarkers for diagnosis and therapeutic targets for human renal diseases. Genomics Inform (2013) 11(4): 224-9. doi:10.5808/GI.2013.11.4.224

439. Mutlu GM, Factor P. Alveolar epithelial $\beta(2)$-adrenergic receptors. Am J Respir Cell Mol Biol (2008) 38(2):127-34. doi:10.1165/rcmb.20070198TR

440. Skeberdis VA. Structure and function of beta3-adrenergic receptors. Medicina (Kaunas) (2004) 40(5):407-13.

441. Billington CK, Ojo OO, Penn RB, Ito S. cAMP regulation of airway smooth muscle function. Pulm Pharmacol Ther (2013) 26(1):112-20. doi:10.1016/ j.pupt.2012.05.007

442. Walker JK, Penn RB, Hanania NA, Dickey BF, Bond RA. New perspectives regarding beta(2) -adrenoceptor ligands in the treatment of asthma. Br J Pharmacol (2011) 163(1):18-28. doi:10.1111/j.1476-5381.2010.01178.x

443. Icard P, Saumon G. Alveolar sodium and liquid transport in mice. Am J Physiol (1999) 277(6 Pt 1):L1232-8.

444. Goodman BE, Brown SE, Crandall ED. Regulation of transport across pulmonary alveolar epithelial cell monolayers. J Appl Physiol Respir Environ Exerc Physiol (1984) 57(3):703-10.

445. Goodman BE, Kim KJ, Crandall ED. Evidence for active sodium transport across alveolar epithelium of isolated rat lung. J Appl Physiol (1985) (1987) 62(6):2460-6.

446. Jayr C, Garat C, Meignan M, Pittet JF, Zelter M, Matthay MA. Alveolar liquid and protein clearance in anesthetized ventilated rats. J Appl Physiol (1985) (1994) 76(6):2636-42.

447. Berthiaume Y, Broaddus VC, Gropper MA, Tanita T, Matthay MA. Alveolar liquid and protein clearance from normal dog lungs. J Appl Physiol (1988) 65(2):585. 
448. Berthiaume Y. Effect of exogenous cAMP and aminophylline on alveolar and lung liquid clearance in anesthetized sheep. J Appl Physiol (1985) (1991) 70(6):2490-7.

449. Norlin A, Finley N, Abedinpour P, Folkesson HG. Alveolar liquid clearance in the anesthetized ventilated guinea pig. Am J Physiol (1998) 274(2 Pt 1): L235-43.

450. Fukuda N, Folkesson HG, Matthay MA. Relationship of interstitial fluid volume to alveolar fluid clearance in mice: ventilated vs. in situ studies. J Appl Physiol (1985) (2000) 89(2):672-9.

451. Sakuma T, Folkesson HG, Suzuki S, Okaniwa G, Fujimura S, Matthay MA. Beta-adrenergic agonist stimulated alveolar fluid clearance in ex vivo human and rat lungs. Am J Respir Crit Care Med (1997) 155(2):506-12. doi:10.1164/ajrccm.155.2.9032186

452. Mutlu GM, Dumasius V, Burhop J, McShane PJ, Meng FJ, Welch L, et al. Upregulation of alveolar epithelial active $\mathrm{Na}+$ transport is dependent on beta2-adrenergic receptor signaling. Circ Res (2004) 94(8):1091-100. doi:10.1161/01.RES.0000125623.56442.20

453. Yue G, Shoemaker RL, Matalon S. Regulation of low-amiloride-affinity sodium channels in alveolar type II cells. Am J Physiol (1994) 267(1 Pt 1): L94-100.

454. Matalon S, O'Brodovich H. Sodium channels in alveolar epithelial cells: molecular characterization, biophysical properties, and physiological significance. Annu Rev Physiol (1999) 61:627-61. doi:10.1146/annurev.physiol. 61.1.627

455. Mutlu GMM, Sznajder JIM. $\beta 2$-agonists for treatment of pulmonary edema: ready for clinical studies? Crit Care Med (2004) 32(7):1607-8. doi:10.1097/ 01.CCM.0000130825.84691.E2

456. Bertorello AM, Ridge KM, Chibalin AV, Katz AI, Sznajder JI. Isoproterenol increases $\mathrm{Na}+\mathrm{K}+$-ATPase activity by membrane insertion of alphasubunits in lung alveolar cells. Am J Physiol (1999) 276(1 Pt 1):L20-7.

457. Lecuona E, Ridge K, Pesce L, Batlle D, Sznajder JI. The GTP-binding protein RhoA mediates Na,K-ATPase exocytosis in alveolar epithelial cells. Mol Biol Cell (2003) 14(9):3888-97. doi:10.1091/mbc.E02-12-0781

458. Minakata Y, Suzuki S, Grygorczyk C, Dagenais A, Berthiaume Y. Impact of beta-adrenergic agonist on $\mathrm{Na}+$ channel and $\mathrm{Na}+\mathrm{K}+$-ATPase expression in alveolar type II cells. Am J Physiol (1998) 275(2 Pt 1):L414-22.

459. Pesce L, Comellas A, Sznajder JI. Beta-adrenergic agonists regulate Na-KATPase via p70S6k. Am J Physiol Lung Cell Mol Physiol (2003) 285(4): L802-7. doi:10.1152/ajplung.00266.2002

460. Berthiaume Y. Long-term stimulation of alveolar epithelial cells by betaadrenergic agonists: increased $\mathrm{Na}+$ transport and modulation of cell growth? Am J Physiol Lung Cell Mol Physiol (2003) 285(4):L798-801. doi:10.1152/ ajplung.00166.2003

461. Suzuki S, Zuege D, Berthiaume Y. Sodium-independent modulation of $\mathrm{Na}(+)-\mathrm{K}(+)$-ATPase activity by beta-adrenergic agonist in alveolar type II cells. Am J Physiol (1995) 268(6 Pt 1):L983-90.

462. Berthiaume Y, Staub NC, Matthay MA. Beta-adrenergic agonists increase lung liquid clearance in anesthetized sheep. JClin Invest (1987) 79(2): 335-43. doi:10.1172/JCI112817

463. Saldias FJ, Lecuona E, Comellas AP, Ridge KM, Rutschman DH, Sznajder JI. beta-adrenergic stimulation restores rat lung ability to clear edema in ventilator-associated lung injury. Am J Respir Crit Care Med (2000) 162(1): 282-7. doi:10.1164/ajrccm.162.1.9809058

464. Dumasius V, Sznajder JI, Azzam ZS, Boja J, Mutlu GM, Maron MB, et al. beta(2)-adrenergic receptor overexpression increases alveolar fluid clearance and responsiveness to endogenous catecholamines in rats. Circ Res (2001) 89(10):907-14. doi:10.1161/hh2201.100204

465. Naren AP, Cobb B, Li C, Roy K, Nelson D, Heda GD, et al. A macromolecular complex of $\beta 2$ adrenergic receptor, CFTR, and ezrin/radixin/moesinbinding phosphoprotein 50 is regulated by PKA. Proc Natl Acad Sci U S A (2003) 100(1):342-6. doi:10.1073/pnas.0135434100

466. Taouil K, Hinnrasky J, Hologne C, Corlieu P, Klossek JM, Puchelle E. Stimulation of beta 2 -adrenergic receptor increases cystic fibrosis transmembrane conductance regulator expression in human airway epithelial cells through a cAMP/protein kinase A-independent pathway. J Biol Chem (2003) 278(19):17320-7. doi:10.1074/jbc.M212227200

467. Berthiaume Y, Folkesson HG, Matthay MA. Lung edema clearance: 20 years of progress: invited review: alveolar edema fluid clearance in the injured lung. J Appl Physiol (1985) (2002) 93(6):2207-13. doi:10.1152/ japplphysiol.01201.2001

468. Gates S, Perkins GD, Lamb SE, Kelly C, Thickett DR, Young JD, et al. Beta-Agonist Lung injury TrIal-2 (BALTI-2): a multicentre, randomised, double-blind, placebo-controlled trial and economic evaluation of intravenous infusion of salbutamol versus placebo in patients with acute respiratory distress syndrome. Health Technol Assess (2013) 17(38):v-vi,1-87. doi:10.3310/hta17380

469. Festic E, Carr GE, Cartin-Ceba R, Hinds RF, Banner-Goodspeed V, Bansal V, et al. Randomized clinical trial of a combination of an inhaled corticosteroid and beta agonist in patients at risk of developing the acute respiratory distress syndrome. Crit Care Med (2017) 45(5):798-805. doi:10.1097/CCM.0000000000002284

470. Briot R, Bayat S, Anglade D, Martiel JL, Grimbert F. Increased cardiac index due to terbutaline treatment aggravates capillary-alveolar macromolecular leakage in oleic acid lung injury in dogs. Crit Care (2009) 13(5):R166. doi:10.1186/cc8137

471. Sylvester JT, Shimoda LA, Aaronson PI, Ward JP. Hypoxic pulmonary vasoconstriction. Physiol Rev (2012) 92(1):367-520. doi:10.1152/physrev. 00041.2010

472. Berthiaume Y, Albertine KH, Grady M, Fick G, Matthay MA. Protein clearance from the air spaces and lungs of unanesthetized sheep over $144 \mathrm{~h}$. J Appl Physiol (1985) (1989) 67(5):1887-97.

473. Friedman M, Vidyasagar R, Joseph N. A randomized, prospective, doubleblind study on the efficacy of dead sea salt nasal irrigations. Laryngoscope (2006) 116(6):878-82. doi:10.1097/01.mlg.0000216798.10007.76

474. Khianey R, Oppenheimer J. Is nasal saline irrigation all it is cracked up to be? Ann Allergy Asthma Immunol (2012) 109(1):20-8. doi:10.1016/j. anai.2012.04.019

475. Mandal MD, Mandal S. Honey: its medicinal property and antibacterial activity. Asian Pac J Trop Biomed (2011) 1(2):154-60. doi:10.1016/S22211691(11)60016-6

476. Angoulvant F, Bellettre X, Milcent K, Teglas JP, Claudet I, Le Guen CG, et al. Effect of nebulized hypertonic saline treatment in emergency departments on the hospitalization rate for acute bronchiolitis: a randomized clinical trial. JAMA Pediatr (2017) 171(8):e171333. doi:10.1001/jamapediatrics.2017.1333

477. Farabaugh KT, Majumder M, Guan BJ, Jobava R, Wu J, Krokowski D, et al. Protein kinase $\mathrm{R}$ mediates the inflammatory response induced by hyperosmotic stress. Mol Cell Biol (2017) 37(4):e521-516. doi:10.1128/MCB. 00521-16

478. Gamboni F, Anderson C, Mitra S, Reisz JA, Nemkov T, Dzieciatkowska M, et al. Hypertonic saline primes activation of the p53-p21 signaling axis in human small airway epithelial cells that prevents inflammation induced by pro-inflammatory cytokines. J Proteome Res (2016) 15(10):3813-26. doi:10.1021/acs.jproteome.6b00602

479. Liu Z, Zhang B, Wang XB, Li Y, Xi RG, Han F, et al. Hypertonicity contributes to seawater aspiration-induced lung injury: role of hypoxia-inducible factor 1alpha. Exp Lung Res (2015) 41(6):301-15. doi:10.3109/01902148. 2015.1030803

480. Machnik A, Neuhofer W, Jantsch J, Dahlmann A, Tammela T, Machura K, et al. Macrophages regulate salt-dependent volume and blood pressure by a vascular endothelial growth factor-C-dependent buffering mechanism. Nat Med (2009) 15(5):545-52. doi:10.1038/nm.1960

481. Kleinewietfeld M, Manzel A, Titze J, Kvakan H, Yosef N, Linker RA, et al. Sodium chloride drives autoimmune disease by the induction of pathogenic TH17 cells. Nature (2013) 496(7446):518-22. doi:10.1038/nature11868

482. Titze J, Shakibaei M, Schafflhuber M, Schulze-Tanzil G, Porst M, Schwind $\mathrm{KH}$, et al. Glycosaminoglycan polymerization may enable osmotically inactive $\mathrm{Na}+$ storage in the skin. Am JPhysiol Heart Circ Physiol (2004) 287(1):H203-8. doi:10.1152/ajpheart.01237.2003

483. Scaife PJ, Mohaupt MG. Salt, aldosterone and extrarenal Na+-sensitive responses in pregnancy. Placenta (2017) 56:53-8. doi:10.1016/j.placenta. 2017.01.100

484. O'Brodovich HM. Immature epithelial $\mathrm{Na}+$ channel expression is one of the pathogenetic mechanisms leading to human neonatal respiratory distress syndrome. Proc Assoc Am Physicians (1996) 108(5):345-55.

485. Helve O, Pitkanen OM, Andersson S, O’Brodovich H, Kirjavainen T, Otulakowski G. Low expression of human epithelial sodium channel in 
airway epithelium of preterm infants with respiratory distress. Pediatrics (2004) 113(5):1267-72. doi:10.1542/peds.113.5.1267

486. Li W, Long C, Renjun L, Zhangxue H, Yin H, Wanwei L, et al. Association of SCNN1A single nucleotide polymorphisms with neonatal respiratory distress syndrome. Sci Rep (2015) 5:17317. doi:10.1038/srep17317

487. De Luca D, van Kaam AH, Tingay DG, Courtney SE, Danhaive O, Carnielli VP, et al. The Montreux definition of neonatal ARDS: biological and clinical background behind the description of a new entity. Lancet Respir Med (2017) 5(8):657-66. doi:10.1016/S2213-2600(17)30214-X

488. Mason JS, Becker JB, Garrity JR. Indications for lung transplantation and patient selection. Lung Transplantation: Principles and Practice. Boca Raton, FL: CRC Press, Taylor \& Francis Group (2016). p. 29-54.

489. Christie JD, Kotloff RM, Pochettino A, Arcasoy SM, Rosengard BR, Landis JR, et al. Clinical risk factors for primary graft failure following lung transplantation. Chest (2003) 124(4):1232-41. doi:10.1378/chest.124. 4.1232

490. Kotloff RM, Keshavjee RM. Lung transplantation. In: Broaddus CV, Mason RJ, Ernst JD, King TE, Lazarus SC, Murray JF, Nadel JA, Slutsky AS, Gotway MB, editors. Murray \& Nadel's Textbook fof Respiratory Medicine. Philadelphia: Elsevier (2016). p. 1832-49.

491. King RC, Binns OA, Rodriguez F, Kanithanon RC, Daniel TM, Spotnitz WD, et al. Reperfusion injury significantly impacts clinical outcome after pulmonary transplantation. Ann Thorac Surg (2000) 69(6):1681-5. doi:10.1016/ S0003-4975(00)01425-9

492. Christie JD, Carby M, Bag R, Corris P, Hertz M, Weill D, et al. Report of the ISHLT working group on primary lung graft dysfunction part II: definition. A consensus statement of the International Society for Heart and Lung Transplantation. J Heart Lung Transplant (2005) 24(10):1454-9. doi:10.1016/j.healun.2004.11.049

493. Christie JD, Kotloff RM, Ahya VN, Tino G, Pochettino A, Gaughan C, et al. The effect of primary graft dysfunction on survival after lung transplantation. Am J Respir Crit Care Med (2005) 171(11):1312-6. doi:10.1164/ rccm.200409-1243OC

494. Thabut G, Mal H, Cerrina J, Dartevelle P, Dromer C, Velly JF, et al. Graft ischemic time and outcome of lung transplantation: a multicenter analysis. Am J Respir Crit Care Med (2005) 171(7):786-91. doi:10.1164/ rccm.200409-1248OC

495. Bharat A, Narayanan K, Street T, Fields RC, Steward N, Aloush A, et al. Early posttransplant inflammation promotes the development of alloimmunity and chronic human lung allograft rejection. Transplantation (2007) 83(2):150-8. doi:10.1097/01.tp.0000250579.08042.b6

496. Whitson BA, Prekker ME, Herrington CS, Whelan TP, Radosevich DM, Hertz MI, et al. Primary graft dysfunction and long-term pulmonary function after lung transplantation. J Heart Lung Transplant (2007) 26(10): 1004-11. doi:10.1016/j.healun.2007.07.018

497. Bharat A, Kuo E, Steward N, Aloush A, Hachem R, Trulock EP, et al. Immunological link between primary graft dysfunction and chronic lung allograft rejection. Ann Thorac Surg (2008) 86(1):189-95. doi:10.1016/ j.athoracsur.2008.03.073; discussion 196-187,

498. Basseri B, Conklin JL, Pimentel M, Tabrizi R, Phillips EH, Simsir SA, et al. Esophageal motor dysfunction and gastroesophageal reflux are prevalent in lung transplant candidates. Ann Thorac Surg (2010) 90(5):1630-6. doi:10.1016/j.athoracsur.2010.06.104

499. Chatterjee S, Nieman GF, Christie JD, Fisher AB. Shear stress-related mechanosignaling with lung ischemia: lessons from basic research can inform lung transplantation. Am J Physiol Lung Cell Mol Physiol (2014) 307(9):L668-80. doi:10.1152/ajplung.00198.2014

500. Cohen DG, Christie JD, Anderson BJ, Diamond JM, Judy RP, Shah RJ, et al. Cognitive function, mental health, and health-related quality of life after lung transplantation. Ann Am Thorac Soc (2014) 11(4):522-30. doi:10.1513/ AnnalsATS.201311-388OC

501. Hartert M, Senbaklavacin O, Gohrbandt B, Fischer BM, Buhl R, Vahld CF. Lung transplantation: a treatment option in end-stage lung disease. Dtsch Arztebl Int (2014) 111(7):107-16. doi:10.3238/arztebl. 2014.0107
502. Huang HJ, Yusen RD, Meyers BF, Walter MJ, Mohanakumar T, Patterson GA, et al. Late primary graft dysfunction after lung transplantation and bronchiolitis obliterans syndrome. Am J Transplant (2008) 8(11):2454-62. doi:10.1111/j.1600-6143.2008.02389.x

503. De Perrot M, Liu M, Waddell TK, Keshavjee S. Ischemia-reperfusioninduced lung injury. Am J Respir Crit Care Med (2003) 167(4):490-511. doi:10.1164/rccm.200207-670SO

504. Tatham KC, O’Dea KP, Romano R, Donaldson HE, Wakabayashi K, Patel BV, et al. Intravascular donor monocytes play a central role in lung transplant ischaemia-reperfusion injury. Thorax (2017) 1-11. doi:10.1136/thoraxjnl-2016208977

505. Keshavjee S, Davis RD, Zamora MR, de Perrot M, Patterson GA. A randomized, placebo-controlled trial of complement inhibition in ischemiareperfusion injury after lung transplantation in human beings. J Thorac Cardiovasc Surg (2005) 129(2):423-8. doi:10.1016/j.jtcvs.2004.06.048

506. Borders C, Ellis J, Cantu EM III, Christie JD. Primary graft dysfunction. In: Vineswaran WT, Garrity JER, Odell JA, editors. Lung transplantation. Principles and Practice. Boca Raton, London, New York: Taylor \& Francis Group (2016). p. 251-63.

507. Cypel M, Keshavjee S. Strategies for safe donor expansion: donor management, donations after cardiac death, ex-vivo lung perfusion. Curr Opin Organ Transplant (2013) 18(5):513-7. doi:10.1097/MOT.0b013e328365191b

508. Juarez MM, Chan AL, Norris AG, Morrissey BM, Albertson TE. Acute exacerbation of idiopathic pulmonary fibrosis-a review of current and novel pharmacotherapies. J Thorac Dis (2015) 7(3):499-519. doi:10.3978/ j.issn.2072-1439.2015.01.17

509. Ryerson CJ, Cottin V, Brown KK, Collard HR. Acute exacerbation of idiopathic pulmonary fibrosis: shifting the paradigm. Eur Respir J (2015) 46(2):512-20. doi:10.1183/13993003.00419-2015

510. Emura I, Usuda H. Acute exacerbation of IPF has systemic consequences with multiple organ injury, with SRA+ and TNF-alpha+ cells in the systemic circulation playing central roles in multiple organ injury. BMC Pulm Med (2016) 16(1):138. doi:10.1186/s12890-016-0298-x

511. Seely EW, Solomon CG. Improving the prediction of preeclampsia. $N$ Engl J Med (2016) 374(1):83-4. doi:10.1056/NEJMe1515223

512. Roberts JM, Lain KY. Recent insights into the pathogenesis of pre-eclampsia. Placenta (2002) 23(5):359-72. doi:10.1053/plac.2002.0819

513. Naeije R, Swenson ER. Inhaled budesonide for acute mountain sickness. Eur Respir J (2017) 50(3). doi:10.1183/13993003.01355-2017

514. Wiedemann HP, Wheeler AP, Bernard GR, Thompson BT, Hayden D, deBoisblanc B, et al. Comparison of two fluid-management strategies in acute lung injury. N Engl J Med (2006) 354(24):2564-75. doi:10.1056/ NEJMoa062200

515. Kuebler WM, Ying X, Singh B, Issekutz AC, Bhattacharya J. Pressure is proinflammatory in lung venular capillaries. JClin Invest (1999) 104(4): 495-502. doi:10.1172/JCI6872

516. Bachofen H, Bachofen M, Weibel ER. Ultrastructural aspects of pulmonary edema. J Thorac Imaging (1988) 3(3):1-7. doi:10.1097/00005382198807000-00005

517. Constantin J-M, Cayot-Constantin S, Roszyk L, Futier E, Sapin V, Dastugue B, et al. Response to recruitment maneuver influences net alveolar fluid clearance in acute respiratory distress syndrome. Anesthesiology (2007) 106(5):944-51. doi:10.1097/01.anes.0000265153.17062.64

Conflict of Interest Statement: The authors declare that the research was conducted in the absence of any commercial or financial relationships that could be construed as a potential conflict of interest.

Copyright $₫ 2018$ Hamacher, Hadizamani, Borgmann, Mohaupt, Männel, Moehrlen, Lucas and Stammberger. This is an open-access article distributed under the terms of the Creative Commons Attribution License (CC BY). The use, distribution or reproduction in other forums is permitted, provided the original author(s) or licensor are credited and that the original publication in this journal is cited, in accordance with accepted academic practice. No use, distribution or reproduction is permitted which does not comply with these terms. 